\title{
Search for new physics at the LHC \& prospects for new discoveries
}

Géraldine Conti (CERN) on behalf of the ATLAS and CMS collaborations

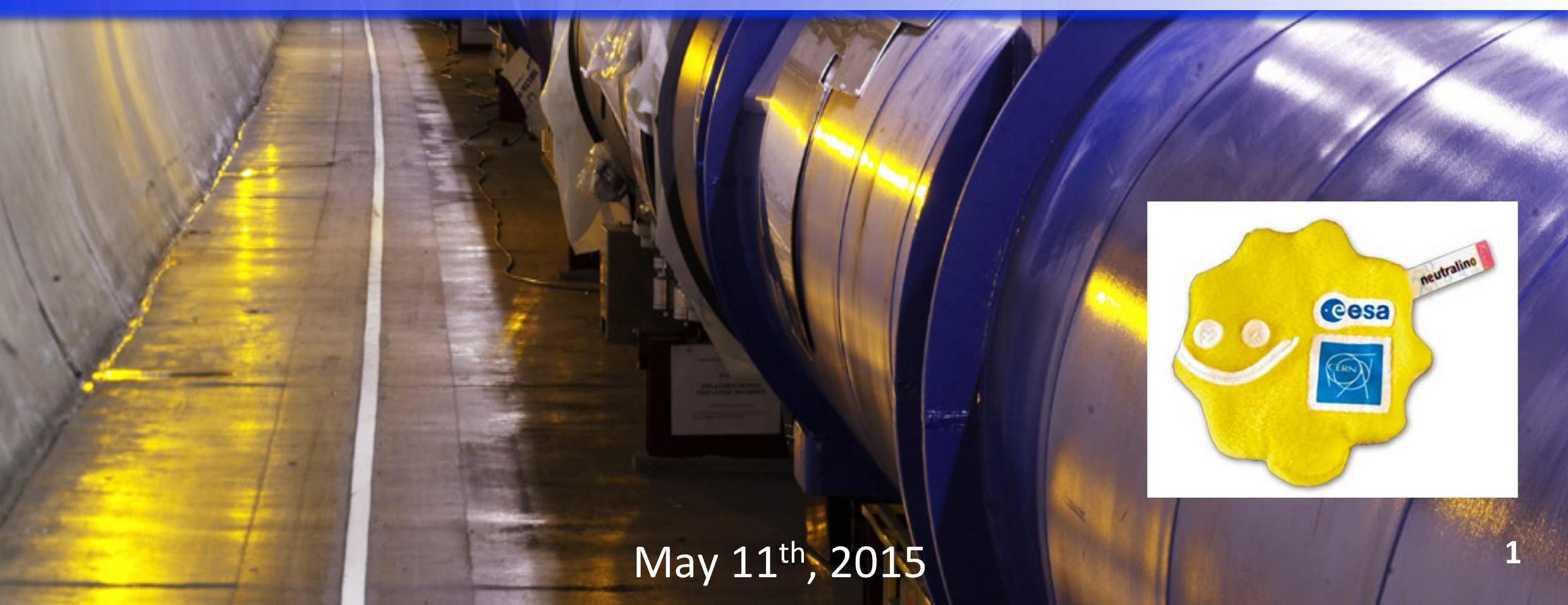




\section{Introduction}

From LHC up to now (2010 - 2012), collision energies of 7 and $8 \mathrm{TeV}$ (Run1) :

- An impressive amount of searches have been carried out in various physics areas

- Disclaimer : not possible to show them all!

- No observation of excesses "Beyond the Standard Model (BSM)" predictions observed

- A few legacy 2-3 $\sigma$ discrepancies remaining

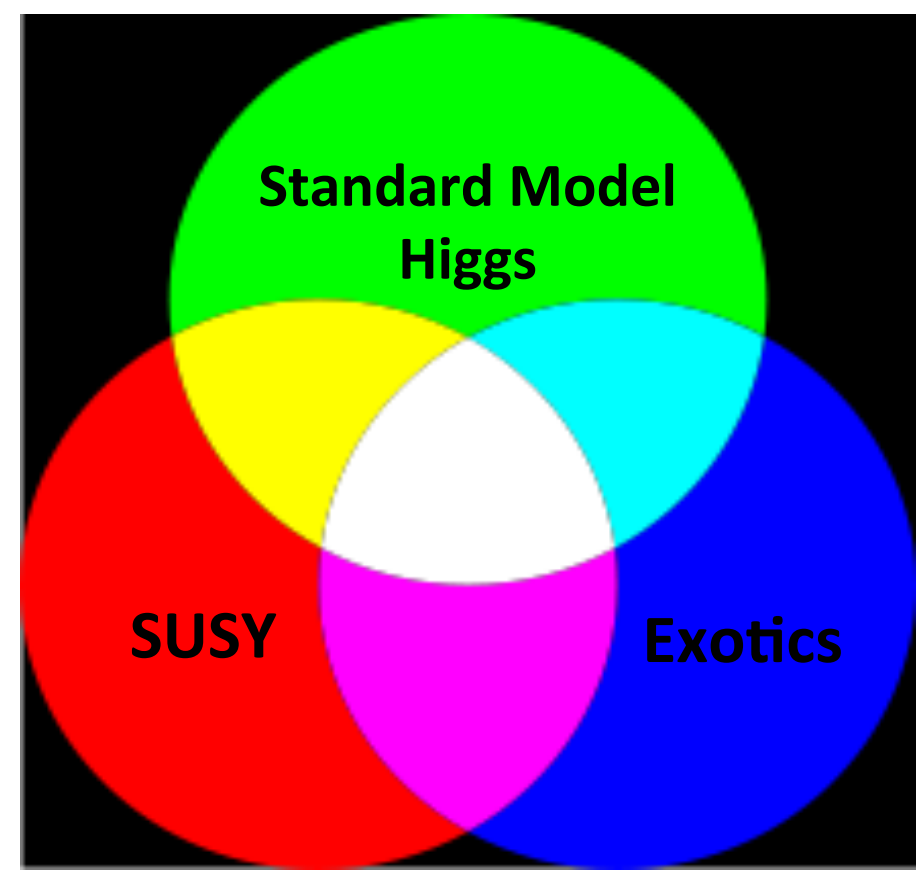

For LHC 2015 - 2018, collision energies of 13 TeV (Run2) :

- An ambitious program to test further the $\mathrm{TeV}$ energy frontier 


\section{LHC, ATLAS and CMS}

- $\mathrm{LHC}$ is a $27 \mathrm{~km}$ proton accelerator and colliding ring at CERN

- The LHC Run1 dataset consists of:

- $2010: \approx 45 \mathrm{pb}^{-1}$ of $\sqrt{ } \mathrm{s}=7 \mathrm{TeV}$ data

- $2011: \approx 5 \mathrm{fb}^{-1}$ of $\sqrt{ } \mathrm{s}=7 \mathrm{TeV}$ data

- $2012: \approx 20 \mathrm{fb}^{-1}$ of $\sqrt{ } \mathrm{s}=8 \mathrm{TeV}$ data

Tevatron got $8 \mathrm{fb}^{-1}$ of $\sqrt{ } \mathrm{s}^{\sim 2} \mathrm{TeV}$ data

- ATLAS and CMS are general

Overall view of the LHC experiments.

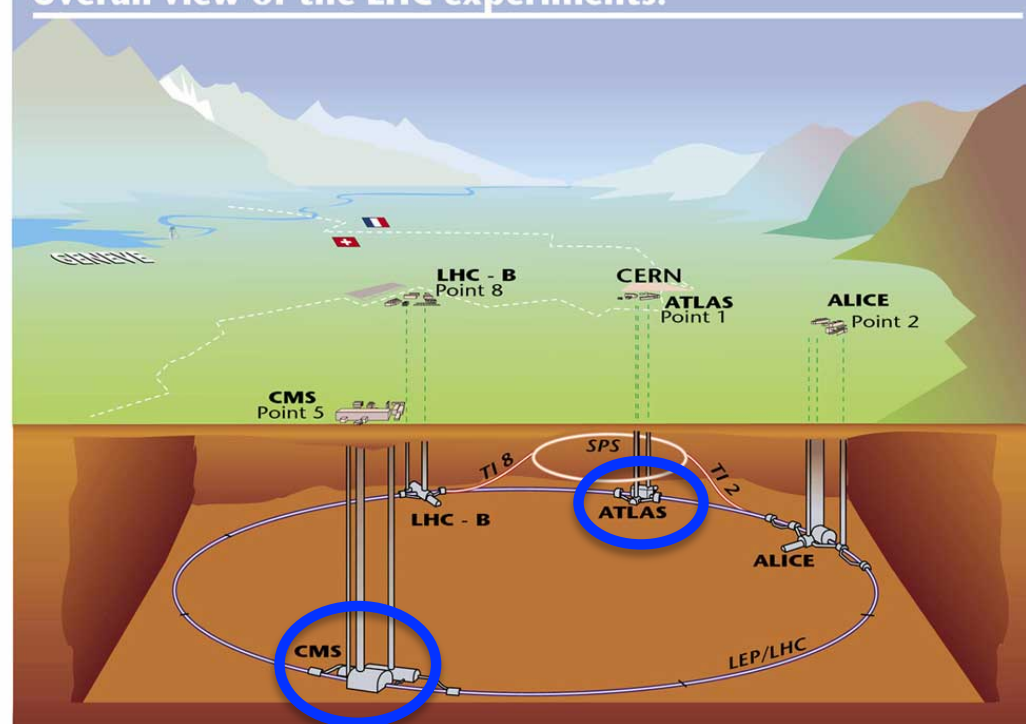
purpose experiments

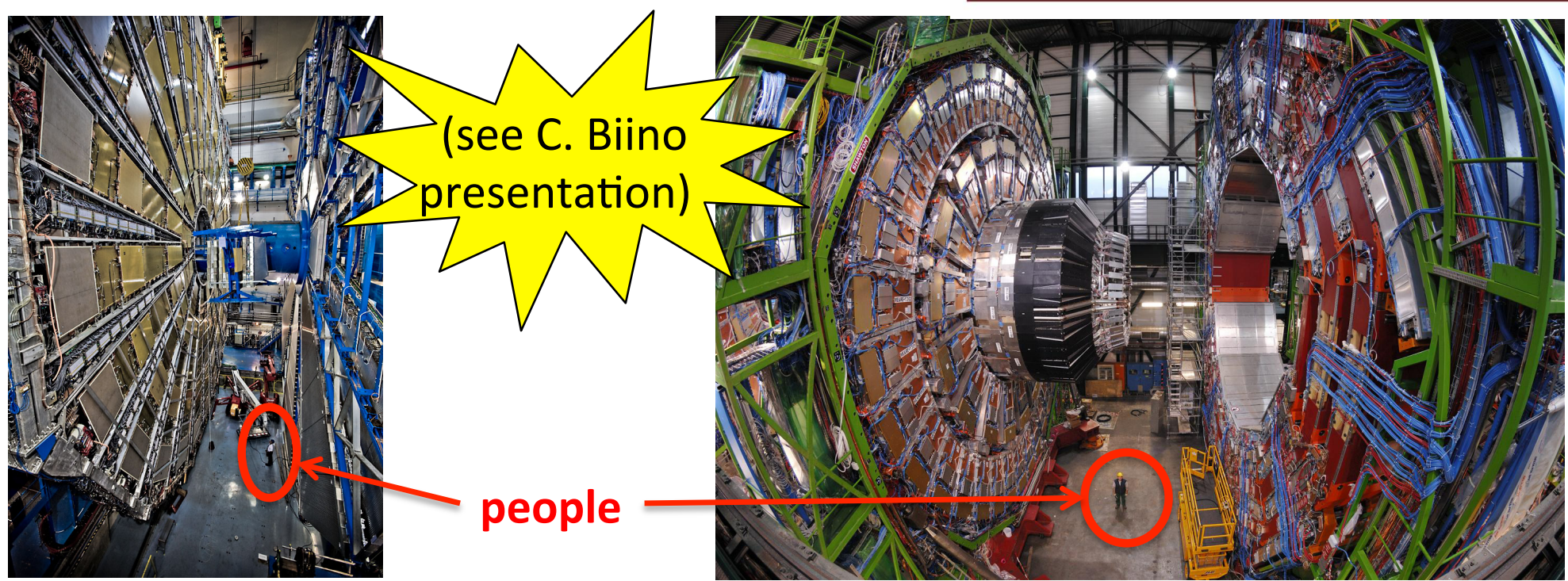




\section{Particle Physics Objects}

\section{Example for $\mathrm{H} \rightarrow \mathrm{ZZ} \rightarrow \mu \mu q q$}

What we calculate :

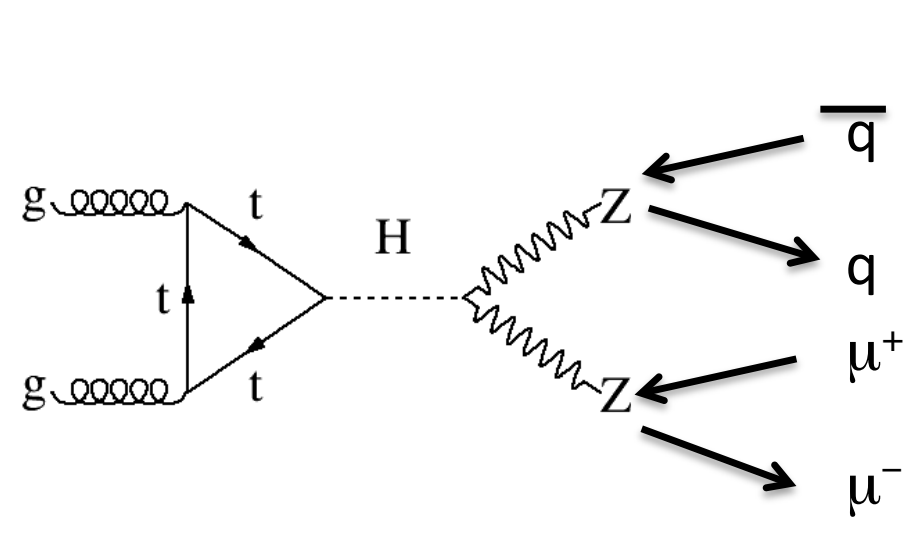
hadrons that are clustered together
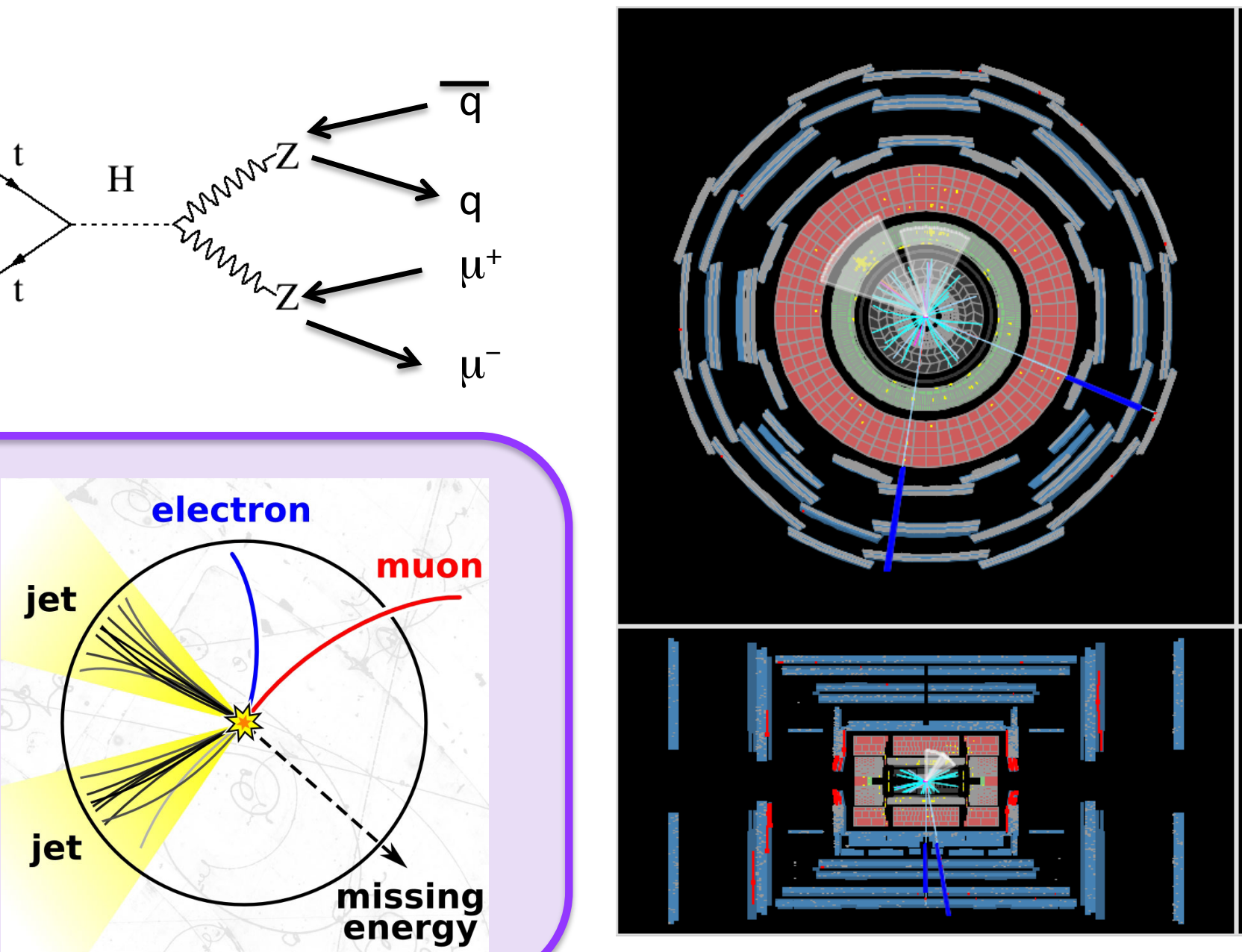

What we measure :

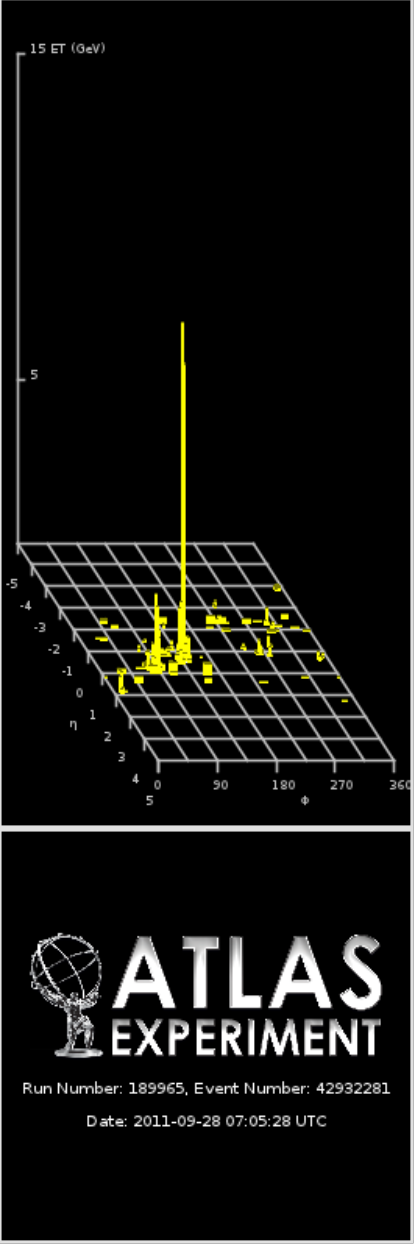




\section{Run1 : BSM Higgs searches}

- The main goal of Run1 to find the Higgs boson was reached:

- In the Higgs sector, searches for BSM physics were performed in :

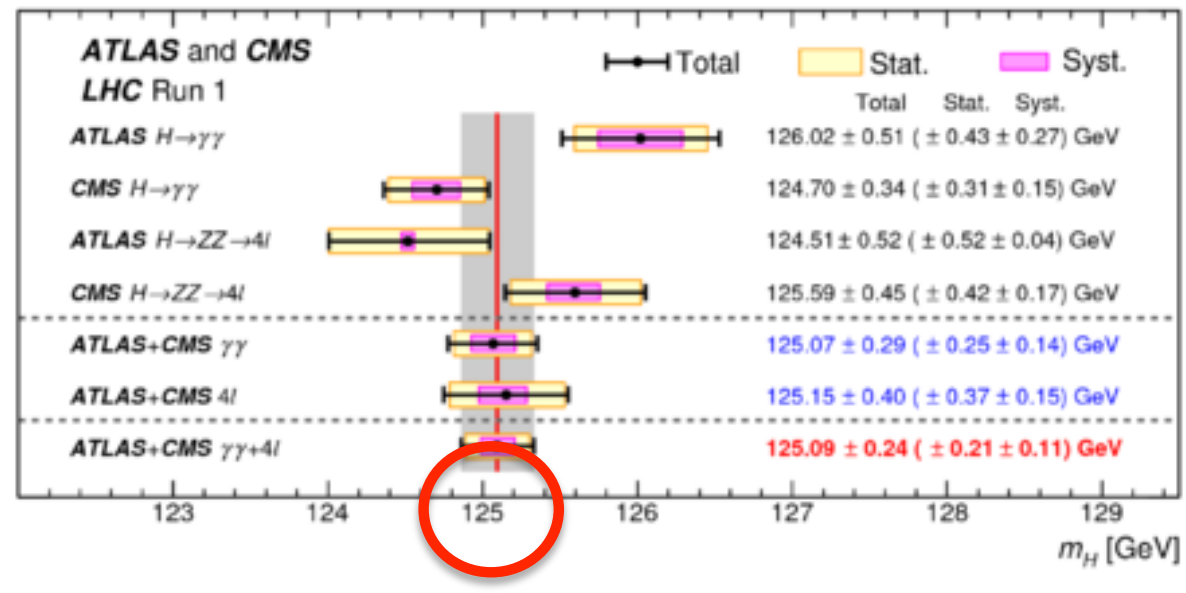

1) New Higgs decays

- Higgs to invisible particles

Two Higgs doublet model (2HDM) :

- Higgs to $\mu \tau$ (lepton flavor violation)

2 CP-even neutral 1 CP-odd 2 charged Higgses: Higgs: Higgses:

2) Other Higgs bosons

$\left(m_{h}, m_{H}, m_{A}, m_{H^{ \pm}}\right)$

- Two Higgs doublet model

- Di-Higgs resonances

- Exotic Higgs

Motivated by SUSY, axion models, possible to generate a baryon asymmetry of the universe of sufficient size 


\section{Search results in BSM Higgs (1)}

- Higgs decaying to invisible particles

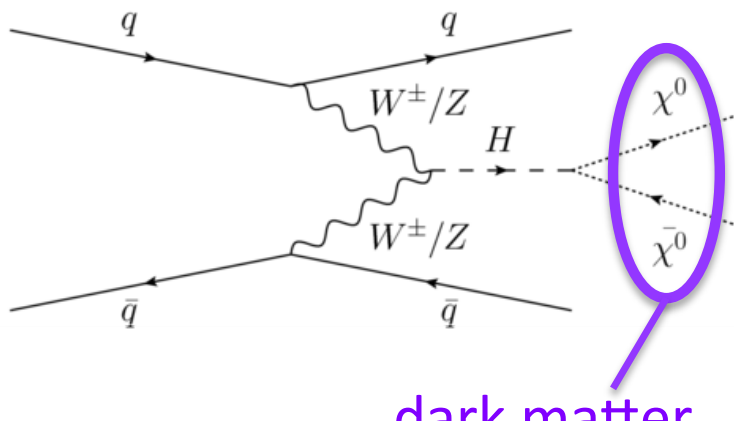
dark matter candidates

\section{Higgs portal model}

Higgs boson acts as mediator between the hidden sector and the SM particles
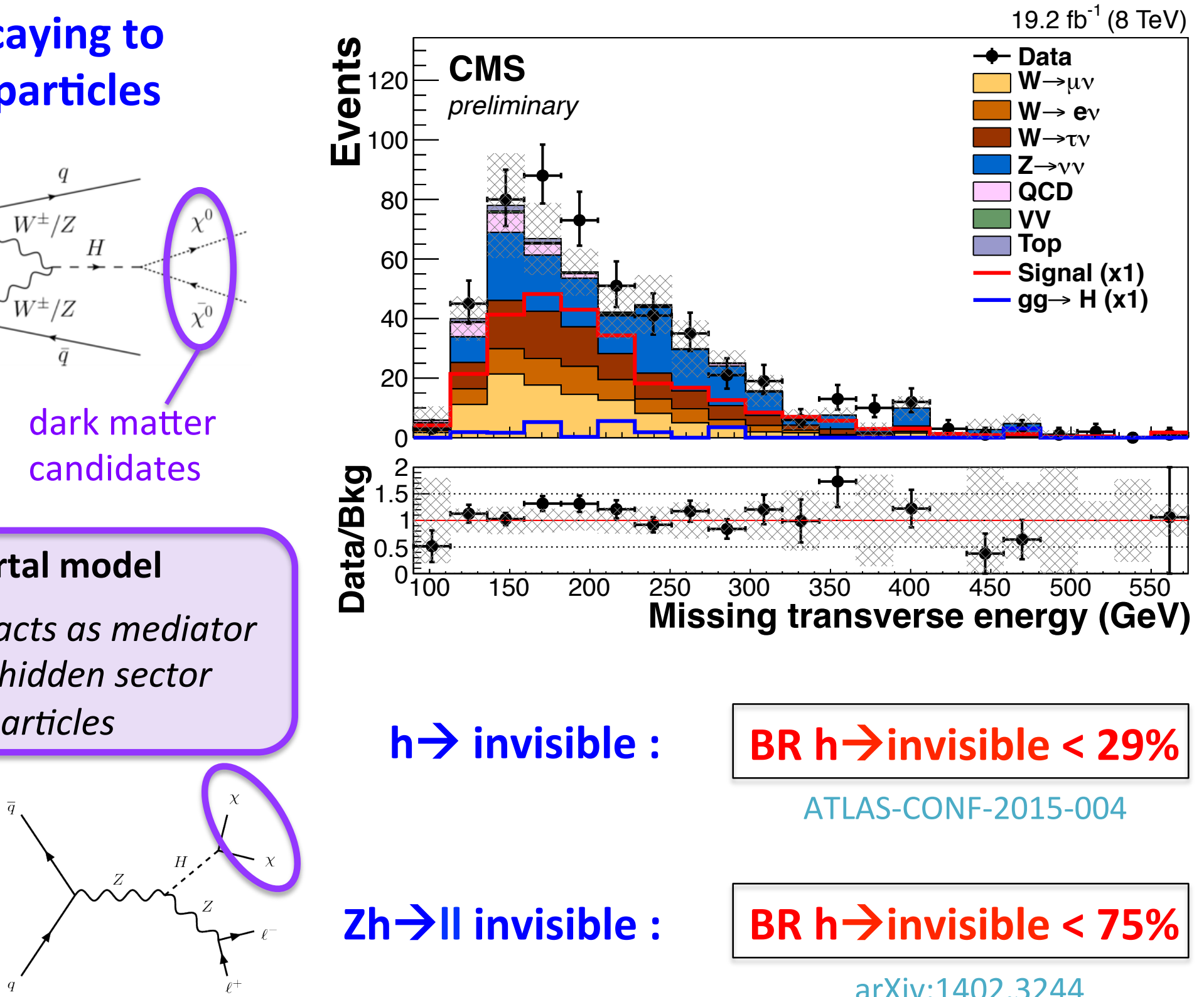

$\mathrm{h} \rightarrow$ invisible :

BR $\mathrm{h} \rightarrow$ invisible $<29 \%$

ATLAS-CONF-2015-004

Zh $\rightarrow$ II invisible :

BR $\mathrm{h} \rightarrow$ invisible $<75 \%$ 


\section{Search results in BSM Higgs (2)}

- Charged Higgs boson

$\mathrm{H}^{+} \rightarrow \mathrm{cs}$
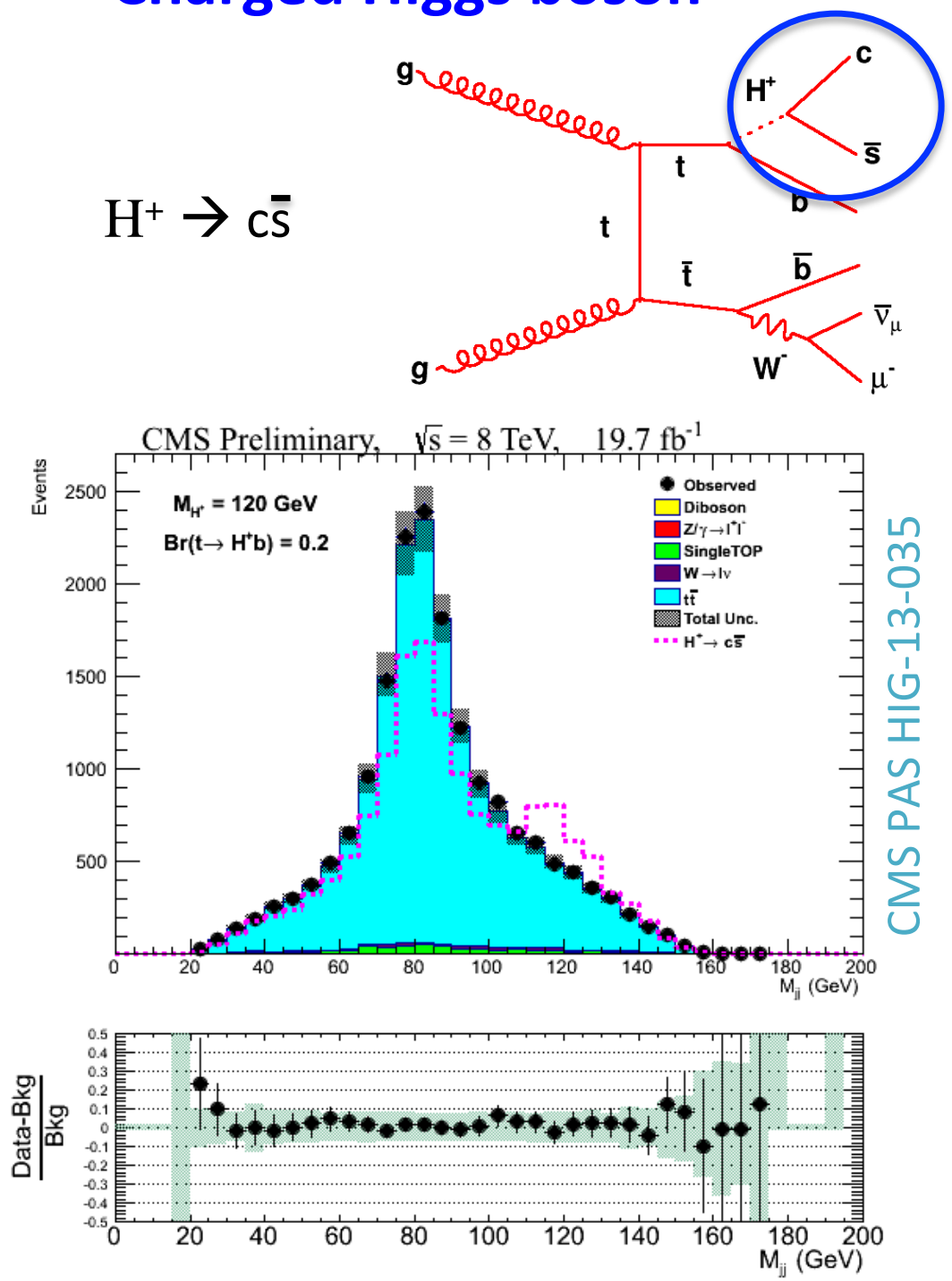

- No significant excess observed

$$
\mathrm{H}^{+} \rightarrow \tau_{\mathrm{had}} \boldsymbol{\nu}
$$

$\tan (\beta)=$ ratio of the two vacuum values of the $2 \mathrm{CP}$-even neutral Higgses $(h, H)$

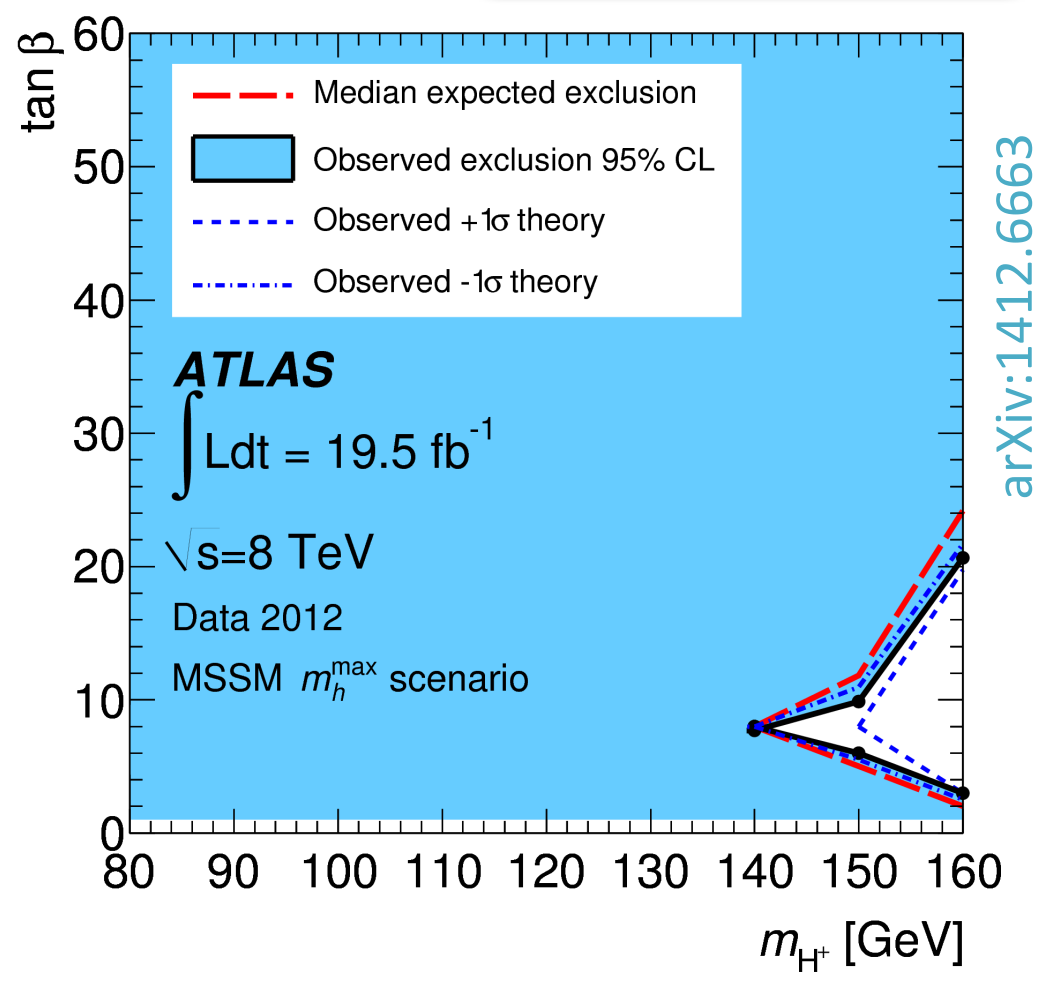

- Stringent limits on $\tan (\beta)$, hence on $2 \mathrm{HDM}$ 


\section{Search results in BSM Higgs (3)}

- CP-odd Higgs A

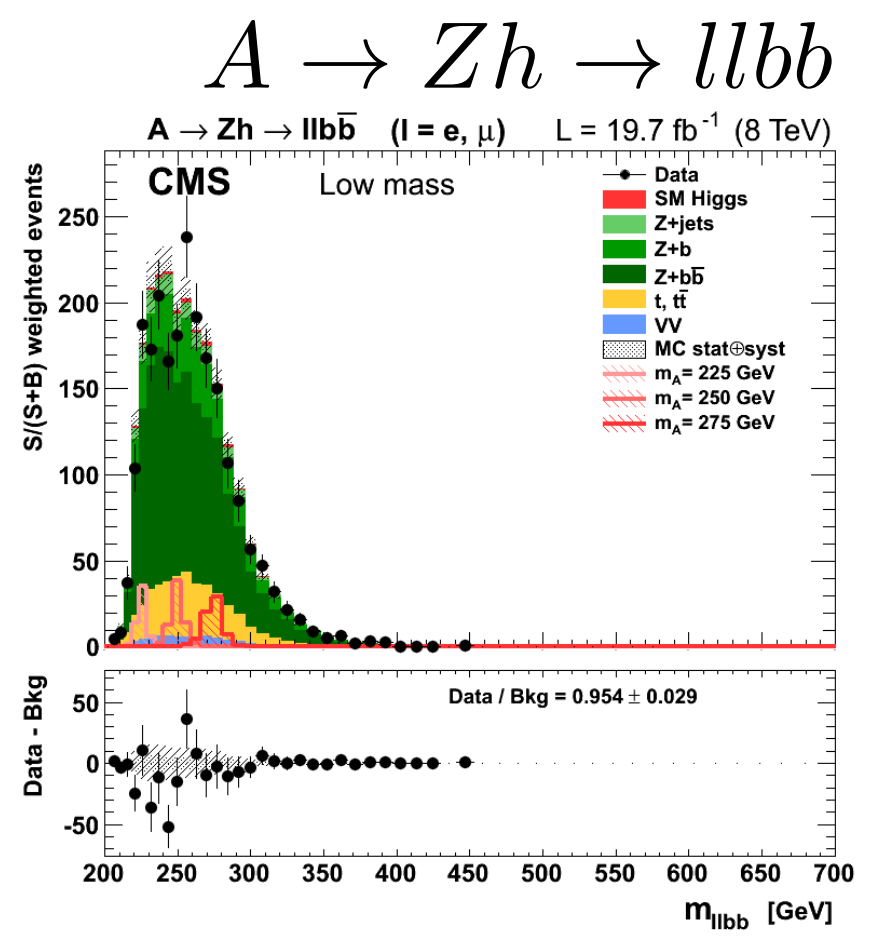

- No excess observed

- di-Higgs resonances

$$
H \rightarrow h h \rightarrow b \bar{b} b \bar{b}(b \bar{b} \gamma \gamma)
$$

Allows to access higher masses $\stackrel{\frac{2}{ \pm}}{\frac{\pi}{ \pm}}$

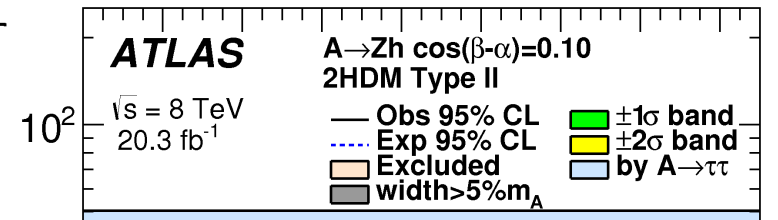

complementarity with $A \rightarrow \tau \tau$ limits

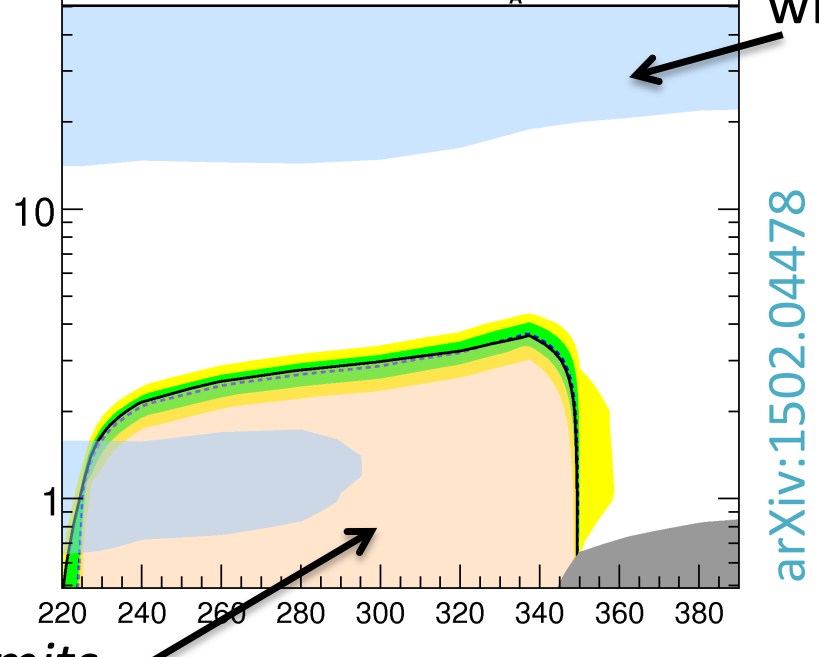

Exclusion limits on $\tan (\beta)$
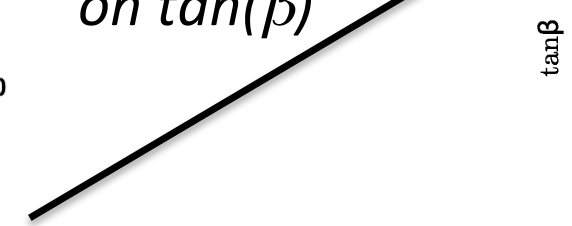


\section{Run1 : SUSY searches}

- Before Run1, supersymmetry (SUSY) was one of the most attractive BSM theories

- Solves the hierarchy problem

( $m_{H}$ unstable at short distances)

- Dark matter candidate (R-parity)

- High-energy unification of weak, strong and EM couplings

\section{SUPERSYMMETRY}

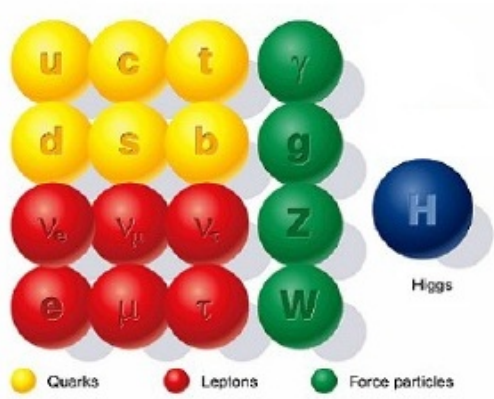

Standard particles

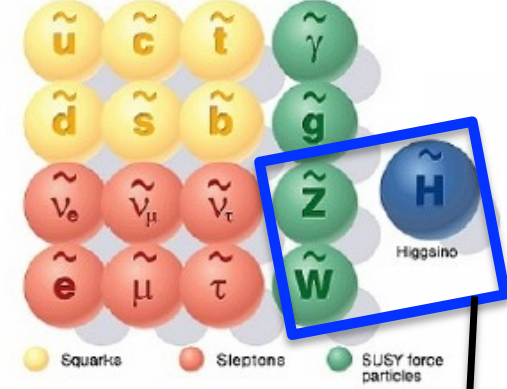

SUSY particles

EW-inos $(\chi)$ are linear combinations of these

- A natural SUSY scenario was favored because it involves a small tuning in the theory.

- Stop needed to solve the hierarchy problem (by cancelling the top loop)

- Gluinos and $3^{\text {rd }}$-generation squarks constrained to be quite light, $\mathrm{O}(\mathrm{TeV}$ ) (Higgs mass at $\approx 125 \mathrm{GeV}$ )

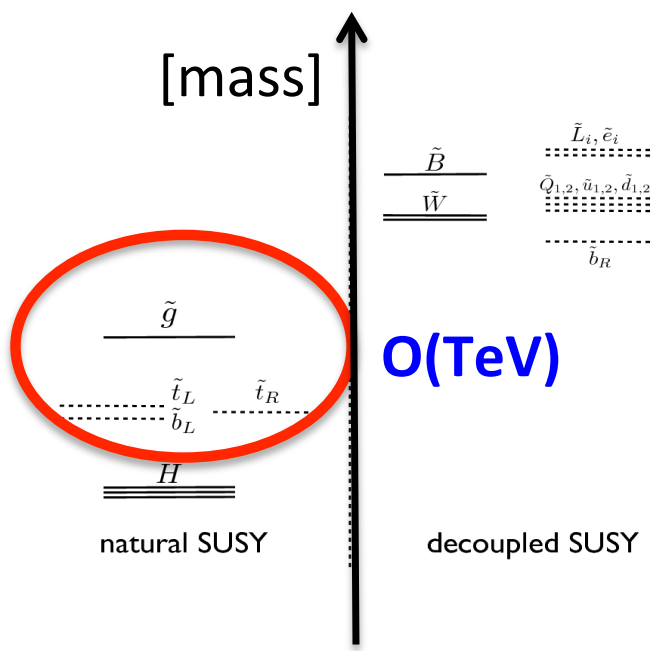

- At LHC, the strategy was to look first for strongly-produced gluinos and $3^{\text {rd }}$-generation squarks

- Should be produced copiously (hadron collider) 


\section{Search results in SUSY (1)}

\section{- Strong production}
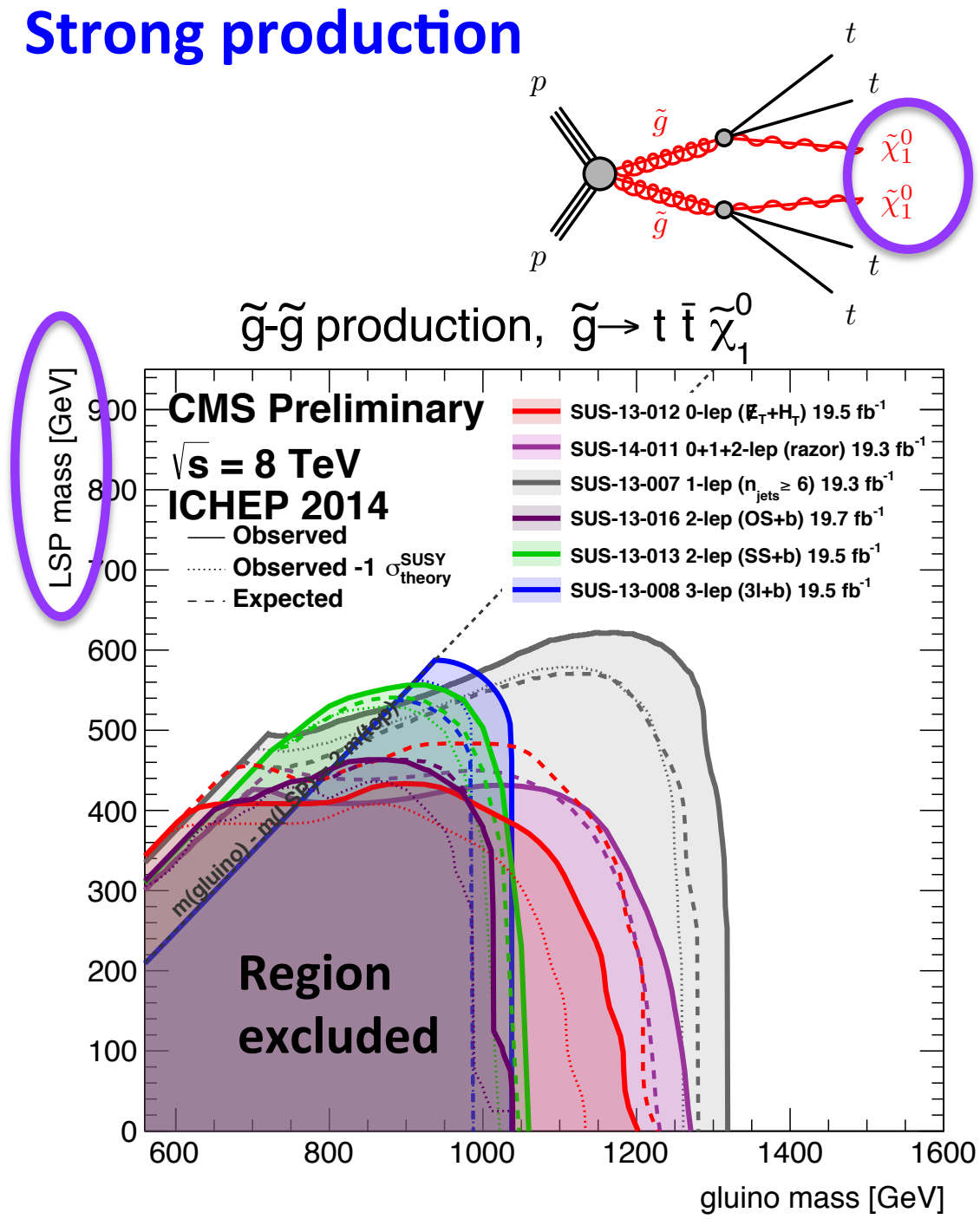

dark matter candidates
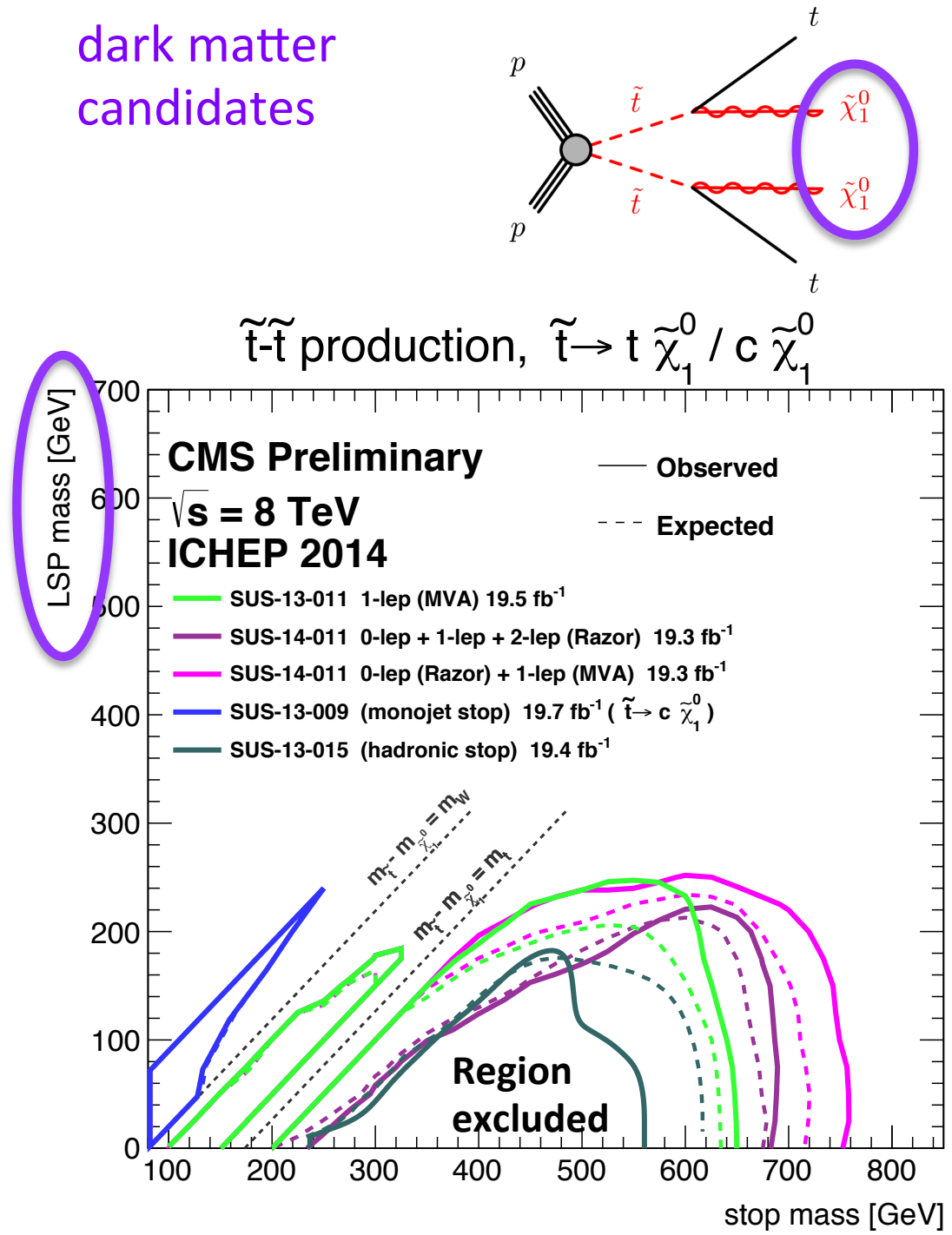

- Limits on gluino mass at $\approx 1.3 \mathrm{TeV}$ (302 GeV pre-LHC), limits on stop mass at $\approx 750 \mathrm{GeV}$ (for $m(L S P)=0 \mathrm{GeV}$ and for the most sensitive scenario) 


\section{Run1 : SUSY searches}

In the absence of strongly-produced SUSY particles in Run1, two alternatives became more popular :

\section{1) electroweak (EWK) production}

Pair-production cross section of SUSY particles:

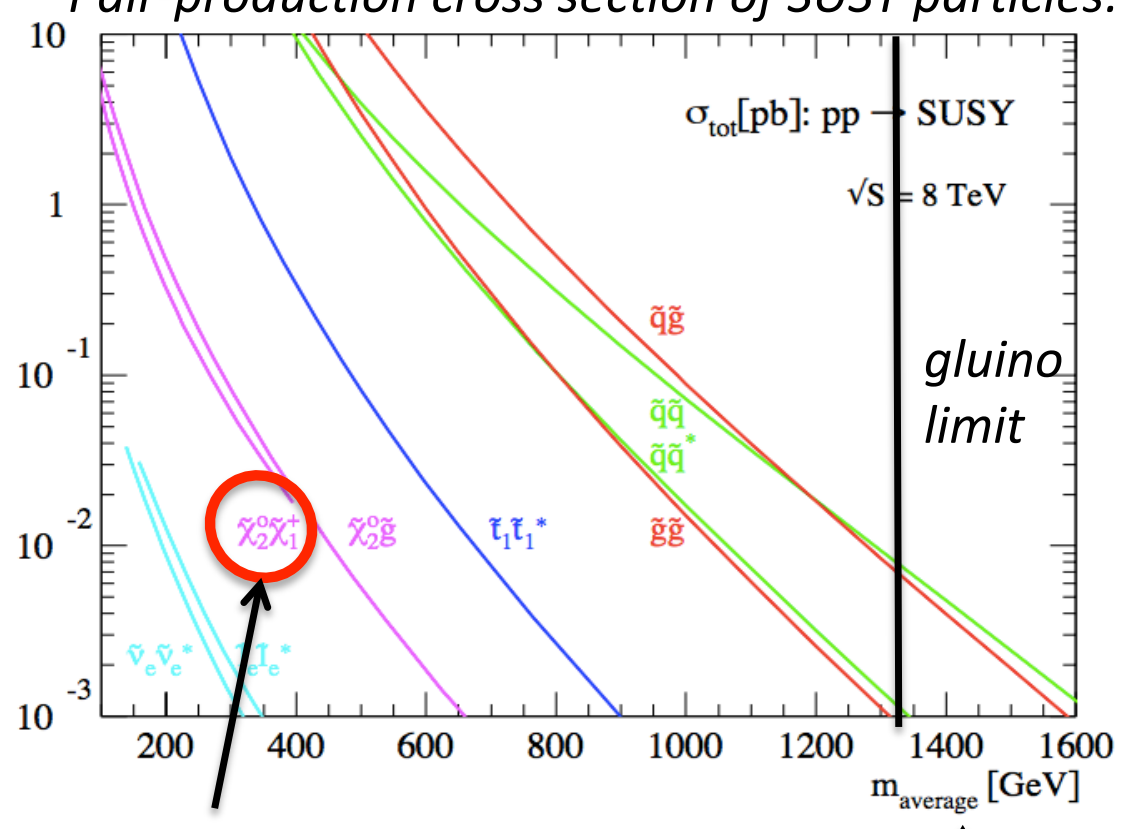

could be the dominant production process at the LHC, hence search for EW-inos pairs
2) Split SUSY

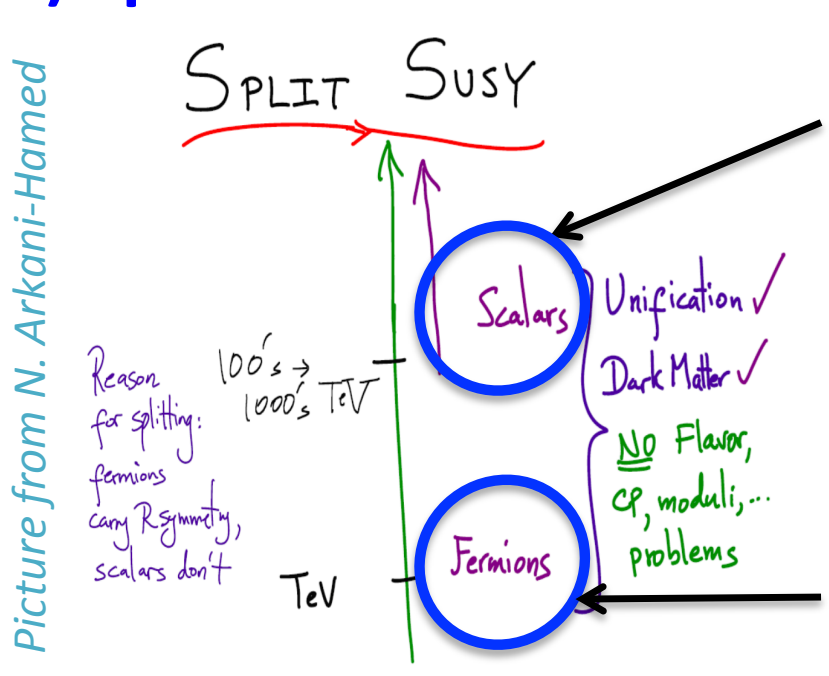

some level of fine tuning accepted, as stop mass can be quite high

only sfermions within LHC reach (gluinos, EW-inos)

Gluino lifetime depends on squark masses $\left(m_{0}\right)$ :

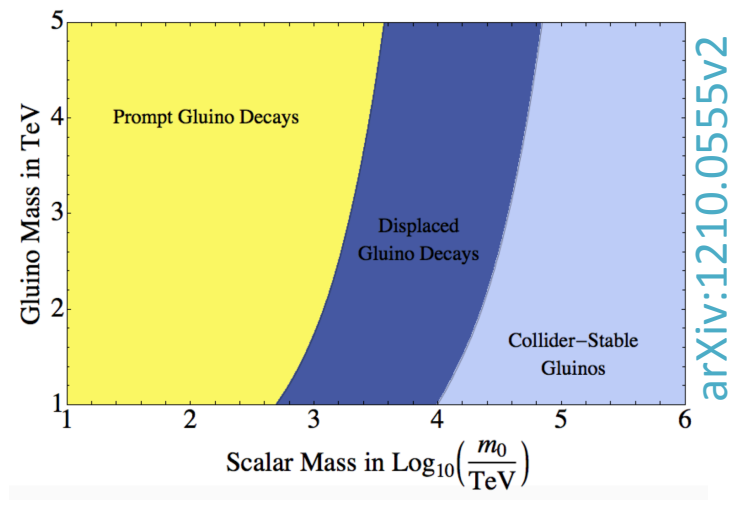

Search for longlived strongly interacting particles 


\section{Search results in SUSY (2)}

\section{- Electroweak production : decays via W/Z bosons or sleptons}

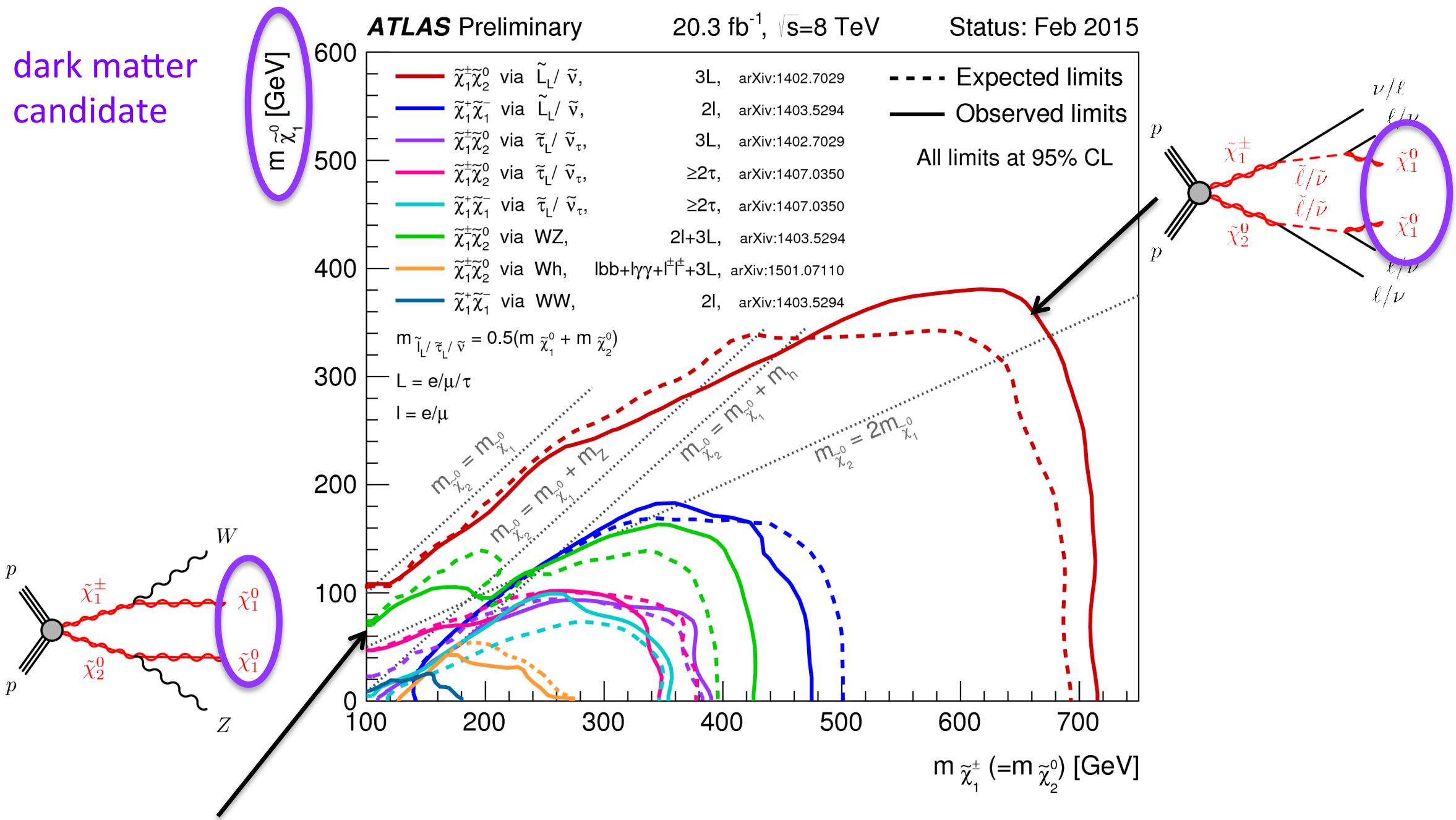

Limit on $\chi_{1}{ }^{0}$ mass up to $\approx 80 \mathrm{GeV}$ (for $\mathrm{m}\left(\chi_{2}{ }^{0} / \chi_{1}{ }^{ \pm}\right)=100 \mathrm{GeV}$ and WZ scenario) 


\section{Search results in SUSY (3)}

\section{- Long-lived particles :}

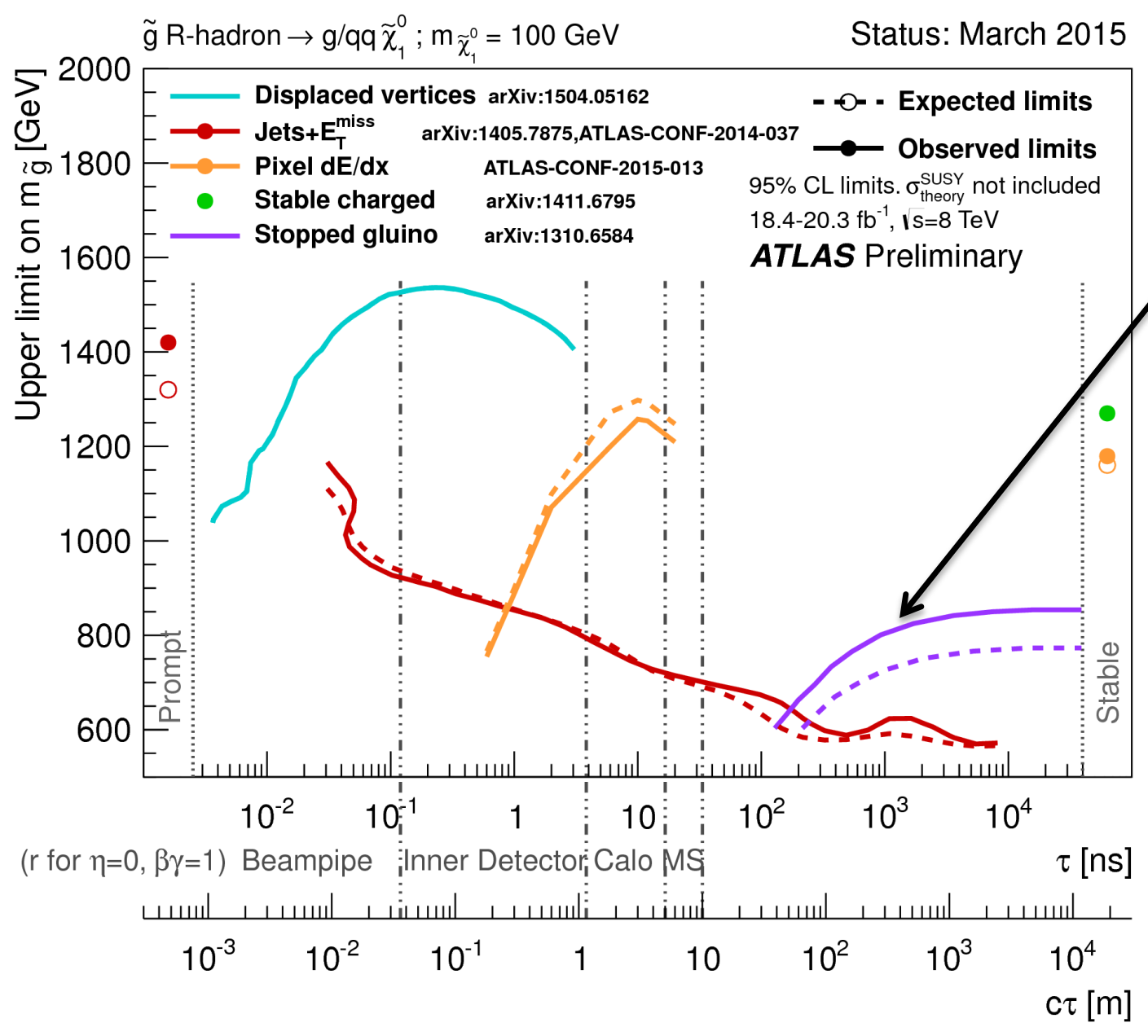

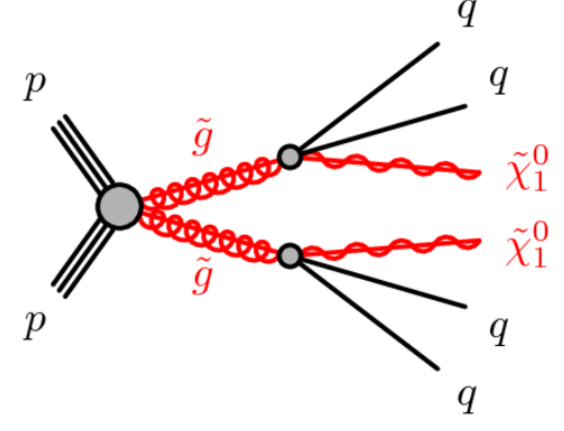

\section{Candidate event display :}

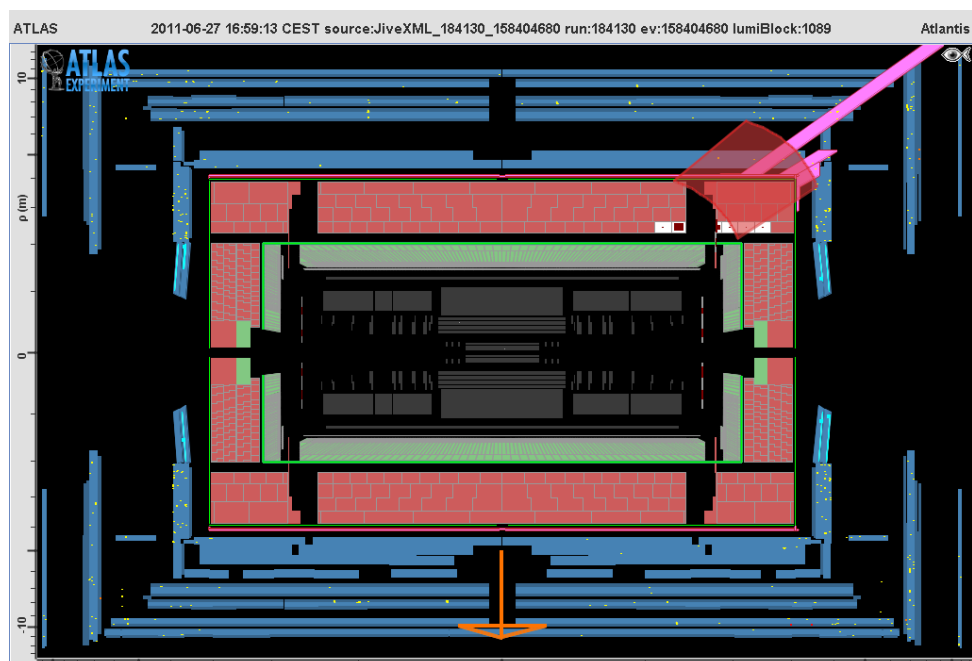

Phys. Rev. D 88, 112003 (2013)

- Nice complementarity of searches covering different lifetimes

- Strongest limit on gluinos with the displaced vertices analysis $(\approx 1550 \mathrm{GeV})$ 


\section{Run1 : Exotics searches}

\begin{tabular}{|c|c|c|c|}
\hline $\begin{array}{l}\text { Leptoquarks (carry both L and } \\
\text { B quantum numbers) } \\
\text { - Appear in various models }\end{array}$ & $\left.\right|_{1} ^{1} \sum_{a}^{g} \xi_{e}^{a Q}$ & $\begin{array}{l}\text { Long-lived particles } \\
\text { - Appear in various models }\end{array}$ & $\begin{array}{l}\text { Example given } \\
\text { for SUSY in } p .13\end{array}$ \\
\hline $\begin{array}{l}\text { RS gravitons } \\
\text { - Randall-Sundrum (RS) model } \\
\text { (explains hierarchy with a } \\
\text { warped extra dimension) }\end{array}$ & $\begin{array}{l}G_{\mathrm{RS}_{1}} \rightarrow \gamma \gamma \\
\text { Di-boson mass } \\
\text { distributions }\end{array}$ & $\begin{array}{l}\text { Dark Matter } \\
\text { - All the models try to } \\
\text { accommodate for it }\end{array}$ & $\begin{array}{l}\text { will be discussed } \\
\text { in p.16 }\end{array}$ \\
\hline $\begin{array}{l}\text { Heavy gauge bosons }\left(W^{\prime}, Z^{\prime}\right) \\
\text { - In models with extended } \\
\text { gauge sectors to achieve } \\
\text { gauge coupling unification }\end{array}$ & $\begin{array}{l}W^{\prime} \rightarrow W Z \\
\text { Di-boson mass } \\
\text { distributions }\end{array}$ & $\begin{array}{l}\text { Large Extra Dimensions } \\
\text { - Appear in string theories } \\
\text { (provides link to gravity } \\
\text { missing in the SM) }\end{array}$ & $\begin{array}{l}\text { Di-lepton mass } \\
\text { distributions }\end{array}$ \\
\hline $\begin{array}{l}\text { Excited fermions } \\
\text { (couple to ordinary SM fermions) } \\
\text { - in compositeness models }\end{array}$ & $l^{*} \rightarrow l Z$ & $\begin{array}{l}\text { Compositeness (Model) } \\
\text { - quarks/leptons are made } \\
\text { of more fundamental } \\
\text { constituents }\end{array}$ & $\begin{array}{l}\text { high energy part } \\
\text { of the di-lepton/ } \\
\text { jet mass spectra }\end{array}$ \\
\hline $\begin{array}{l}\text { Multijet resonances } \\
\text { - Appear in various models }\end{array}$ & $\begin{array}{l}\text { Events with } \\
\text { lots of jets }\end{array}$ & $\begin{array}{l}\text { (addresses the number of } \\
\text { quark/lepton generation, } \\
\text { charges and masses) }\end{array}$ & (non-resonant) \\
\hline
\end{tabular}

+ searches for Higgs resonances (Vh with $V=W, Z$ ) 


\section{Search results in Exotics}

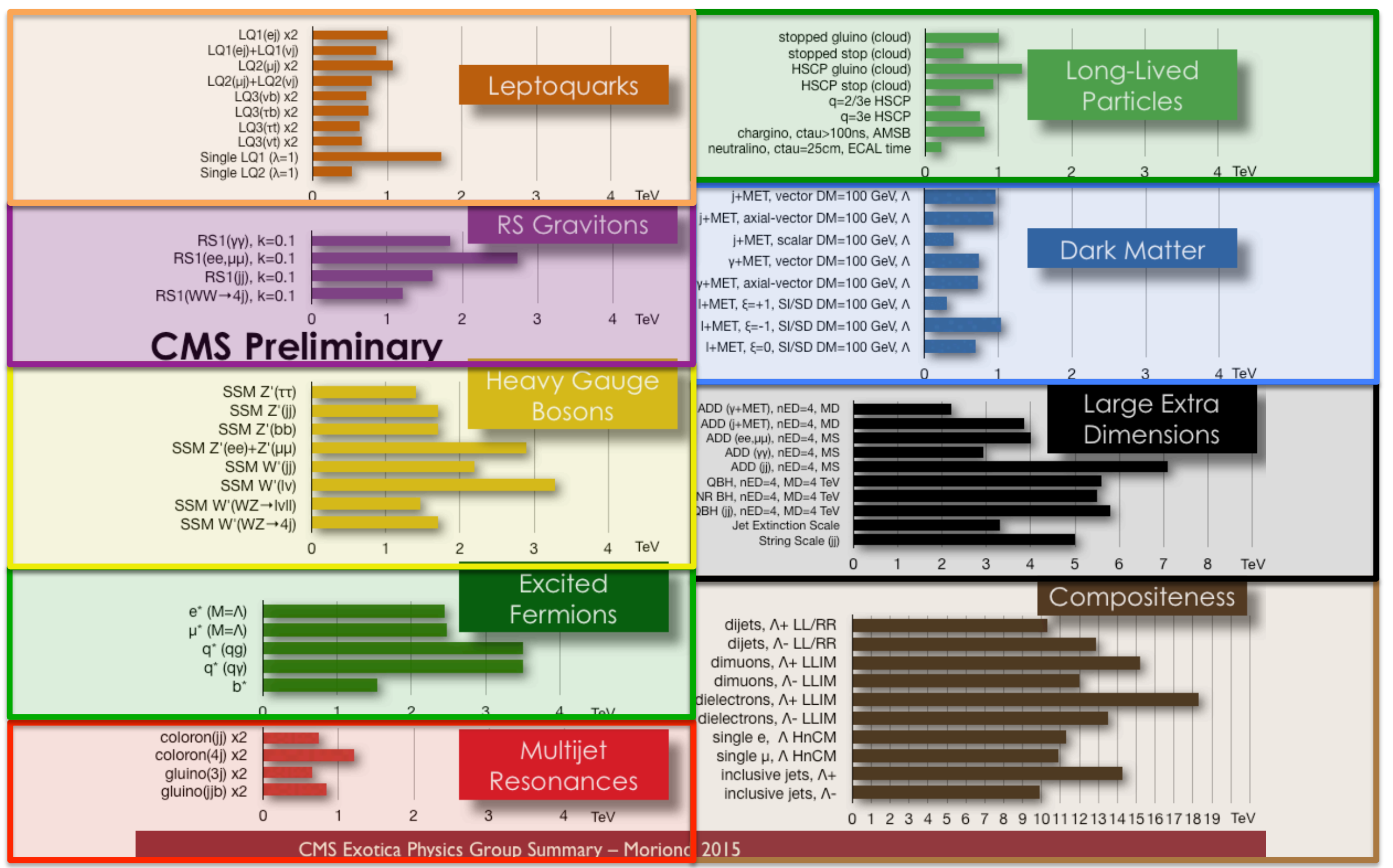

https://twiki.cern.ch/twiki/pub/CMSPublic/PhysicsResultsCombined/exo-limits_Moriond2015.pdf 


\section{Dark Matter Detection}

- Dark Matter creation

If WIMP interactions are sensitive to the spin of the nucleus, the cross section is spin-dependent. If not, the cross section is spinindependent

- At the LHC, look for an energetic object and missing energy

Energetic jet :

Energetic photon :

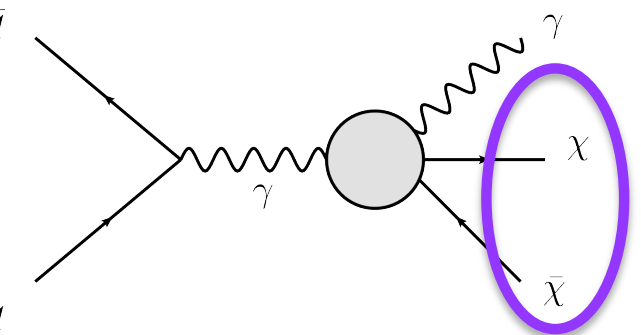

\section{Production \\ Indirect \\ Direct}

$p+\bar{p} \rightarrow X+X \quad X+X \rightarrow p+\bar{p} \quad X+p \rightarrow X+p$
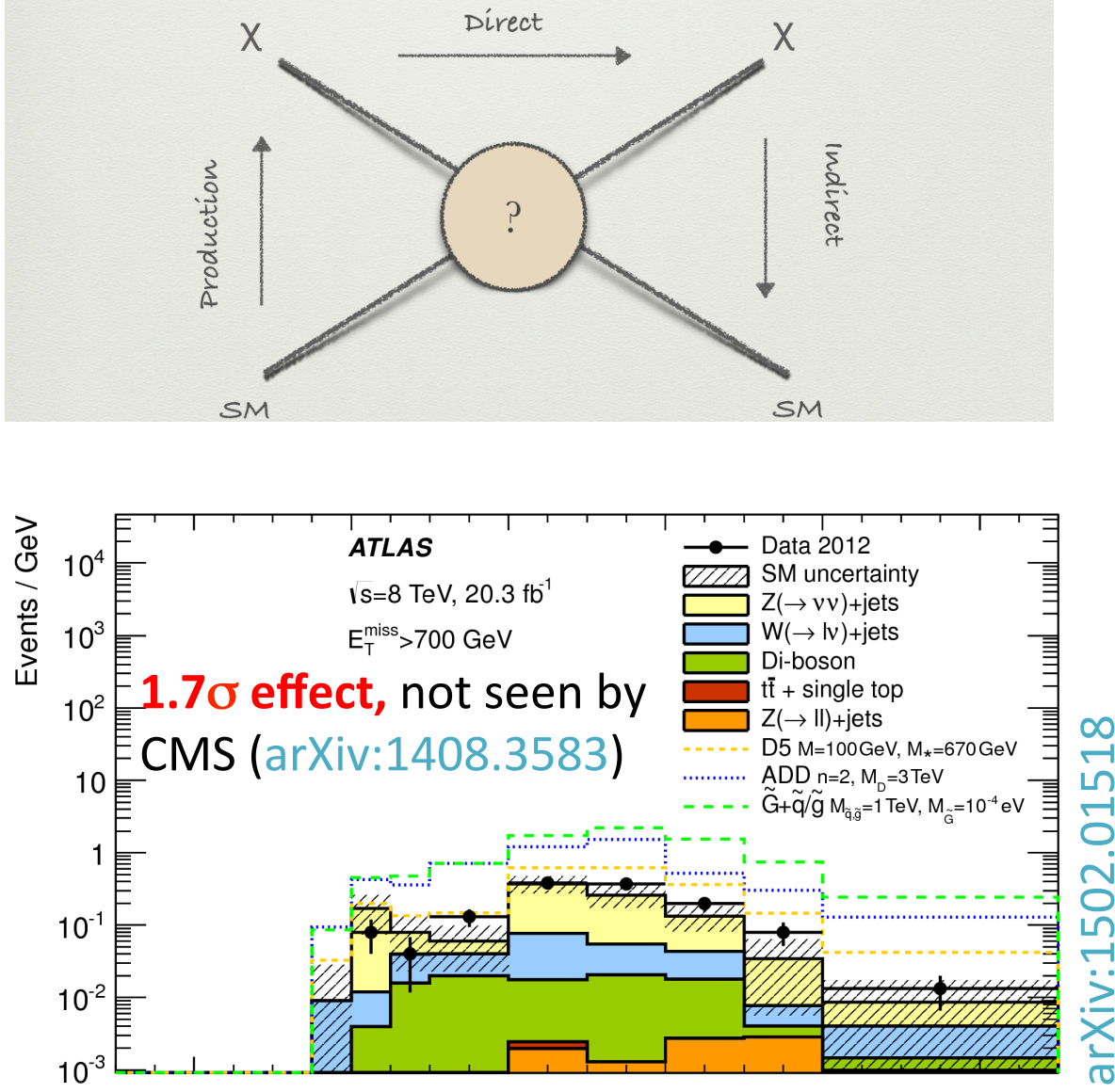

离

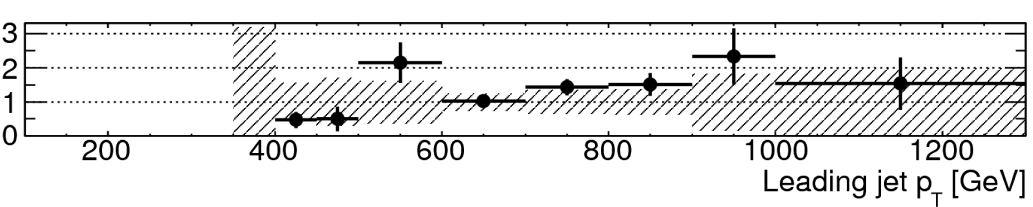




\section{Effective Field Theory (EFT) approach}

- SM-WIMP coupling via a contact interaction :

- $\mathrm{M}_{\text {med }}=$ mass of the mediator

- $\mathrm{Q}_{\mathrm{tr}}=$ scale of the interaction (=invariant mass of the two $\chi$ )

- Condition : $\mathrm{M}_{\text {med }}>\mathrm{Q}_{\mathrm{tr}}$

- to ensure that the mediator cannot be produced directly in LHC collisions and can be integrated out with an EFT formalism

- in the next slide : truncated = remove events that do not satisfy the condition

- $\mathrm{M}_{\text {med }}$ depends on the couplings of the mediator to the SM/DM particles

- Interaction couplings : WIMPs-SM

WIMPs = scalars

WIMPs = Dirac fermions

\begin{tabular}{|c|c|c|c|}
\hline Name & Initial state & Type & Operator \\
\hline C1 & $q q$ & scalar & $\frac{m_{q}}{M_{*}^{2}} \chi^{\dagger} \chi \bar{q} q$ \\
\hline C5 & $g g$ & scalar & $\frac{1}{4 M_{*}^{2}} \chi^{\dagger} \chi \alpha_{\mathrm{s}}\left(G_{\mu \nu}^{a}\right)^{2}$ \\
\hline D1 & $q q$ & scalar & $\frac{m_{q}}{M_{\star}^{3}} \bar{\chi} \chi \bar{q} q$ \\
\hline D5 & $q q$ & vector & $\frac{1}{M_{\star}^{2}} \bar{\chi} \gamma^{\mu} \chi \bar{q} \gamma_{\mu} q$ \\
\hline D8 & $q q$ & axial-vector & $\frac{1}{M_{\star}^{2}} \bar{\chi} \gamma^{\mu} \gamma^{5} \chi \bar{q} \gamma_{\mu} \gamma^{5} q$ \\
\hline D9 & $q q$ & tensor & $\frac{1}{M_{\star}^{2}} \bar{\chi} \sigma^{\mu \nu} \chi \bar{q} \sigma_{\mu \nu} q$ \\
\hline D11 & $g g$ & scalar & $\frac{1}{4 M_{*}^{3}} \bar{\chi} \chi \alpha_{\mathrm{s}}\left(G_{\mu \nu}^{a}\right)^{2}$ \\
\hline
\end{tabular}

- EFT allows for direct comparison with (in)direct DM results

- improvements expected for Run2 http://moriond.in2p3.fr/QCD/2015/WednesdayAfternoon/Landsberg.pdf 


\section{DM Search Results (1)}

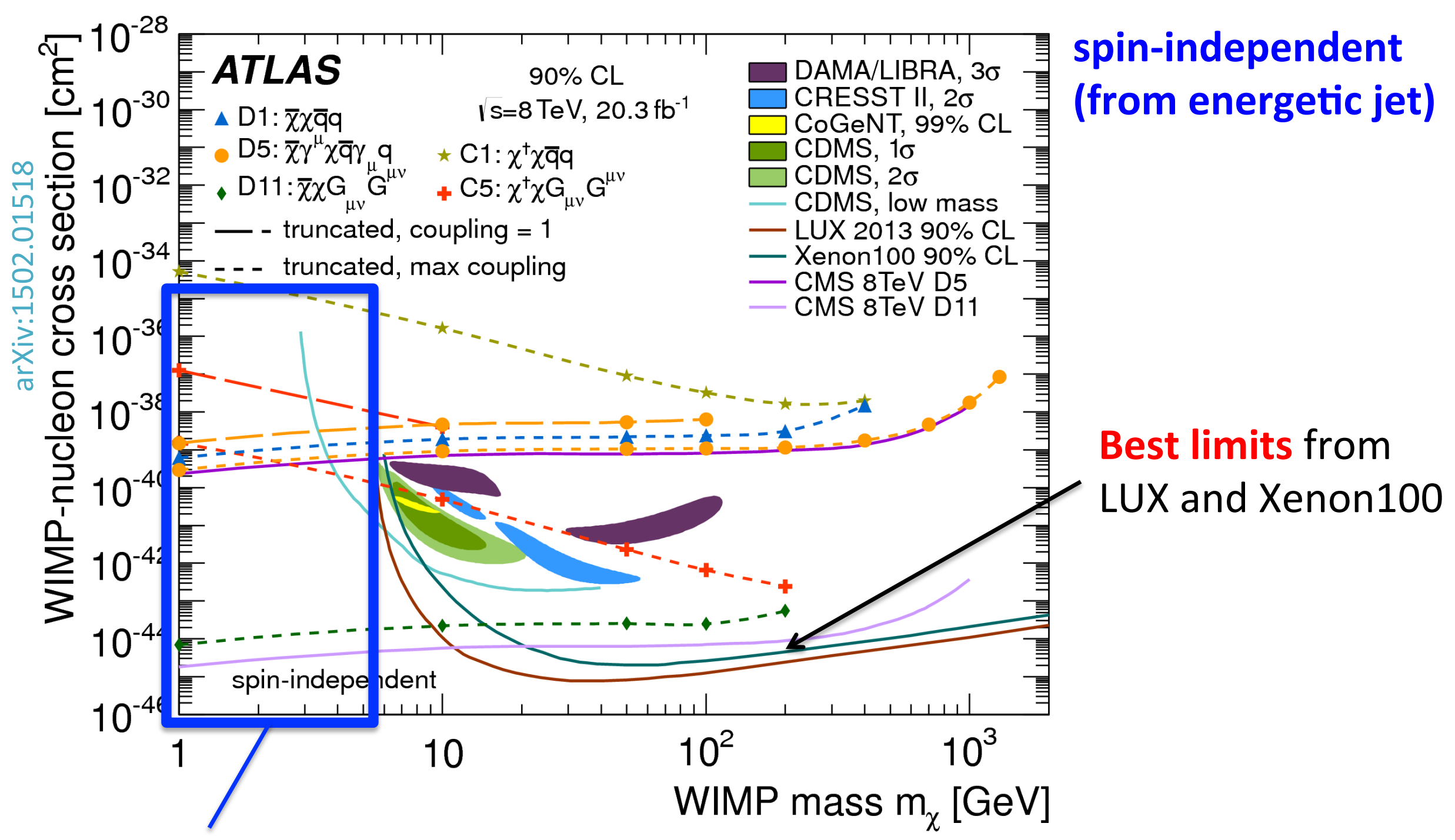

LHC allows for access to low-mass WIMP scenario, which is nicely complementary to other types of experiments 


\section{DM Search Results (3)}

From (Zh $\rightarrow$ Il invisible) analysis shown in page 6: arXiv:1402.3244

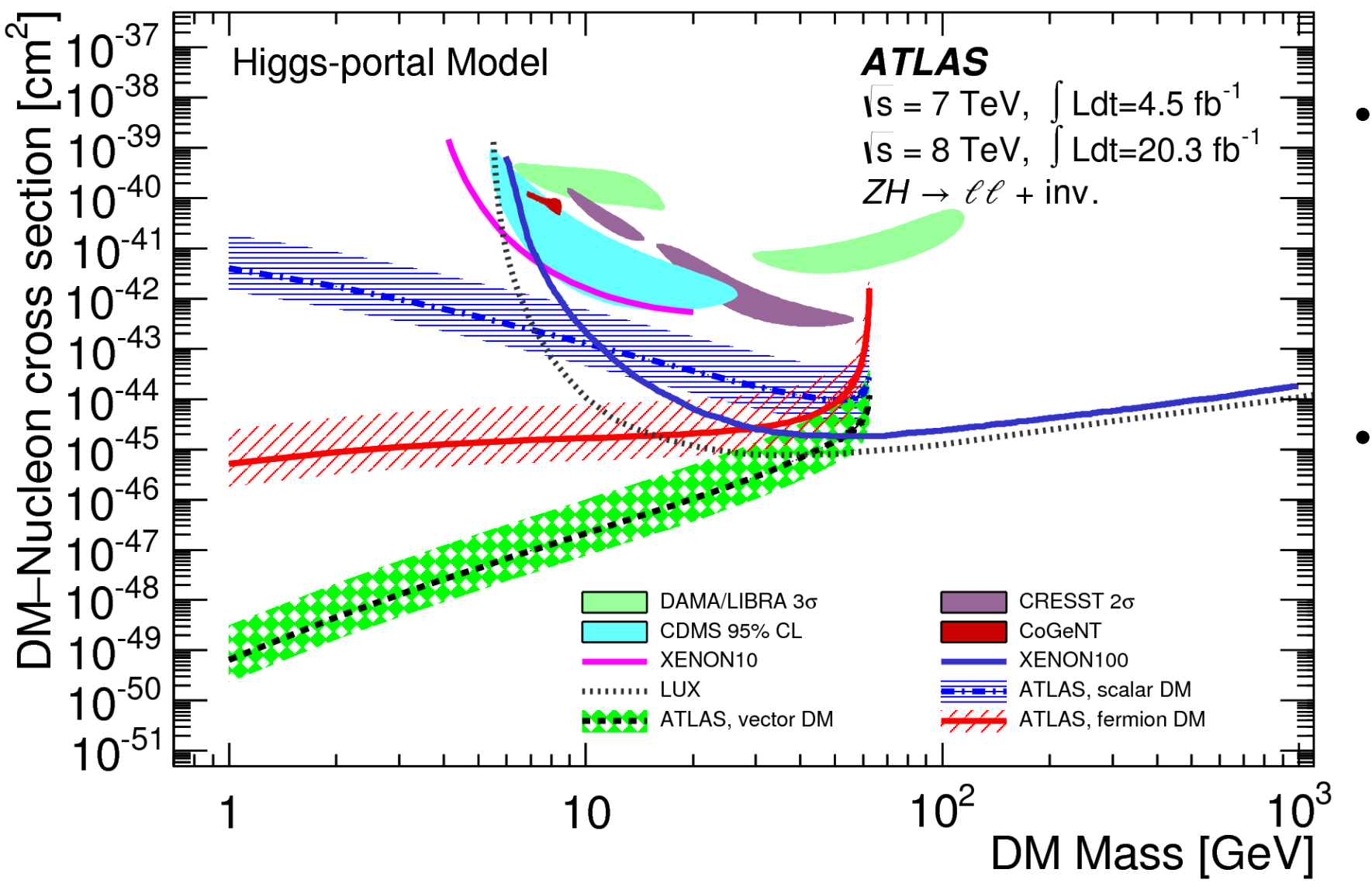

\section{Higgs portal model}

- WIMPs of different nature considered (scalar, vector or fermion)

- Direct detection : spinindependent results from searches for nuclei recoils from elastic scattering of WIMPs

LHC has the best limits for WIMP masses below $m_{H} / 2$ (vector DM) 


\section{Run1 : A few small excesses left (1)}

As of May $11^{\text {th }}, 2015$ :

\begin{tabular}{|c|c|c|}
\hline Areas & ATLAS & CMS \\
\hline \multirow[t]{3}{*}{ Higgs } & $-\mathrm{H} \rightarrow \mathrm{hh} \rightarrow$ bbvv (back-up) & - Higgs to invisible (p.6) \\
\hline & $-\mathrm{ttH}$ & $-\mathrm{ttH}$ \\
\hline & & - LVF H $\rightarrow \tau \mu$ (back-up) \\
\hline \multirow[t]{2}{*}{ SUSY } & $-\mathrm{Z}+\mathrm{MET}$ & - Di-lepton mass edge \\
\hline & & - Multilepton $3 \mid+\tau$ (back-up) \\
\hline \multirow[t]{4}{*}{ Exotics } & - Dark Matter (p.16) & - Di-jet mass search \\
\hline & - Same-sign + b-jets & - Di-lepton mass search \\
\hline & \multirow{2}{*}{$\begin{array}{l}\text { - Type III Seesaw heavy } \\
\text { leptons (back-up) }\end{array}$} & - Di-boson mass search \\
\hline & & $\begin{array}{l}\text { - Heavy neutrinos and right-handed } \\
\text { W bosons }\end{array}$ \\
\hline
\end{tabular}

- Analyses grouped together with color codes will be presented together in the following. For others, you can find information in the back-up slides. 


\section{SUSY Excesses (2)}

\section{2 leptons (on/off-Z) + jets + missing energy}

- ATLAS has an excess $\approx$ at the mass of the $Z$ for the electron channel

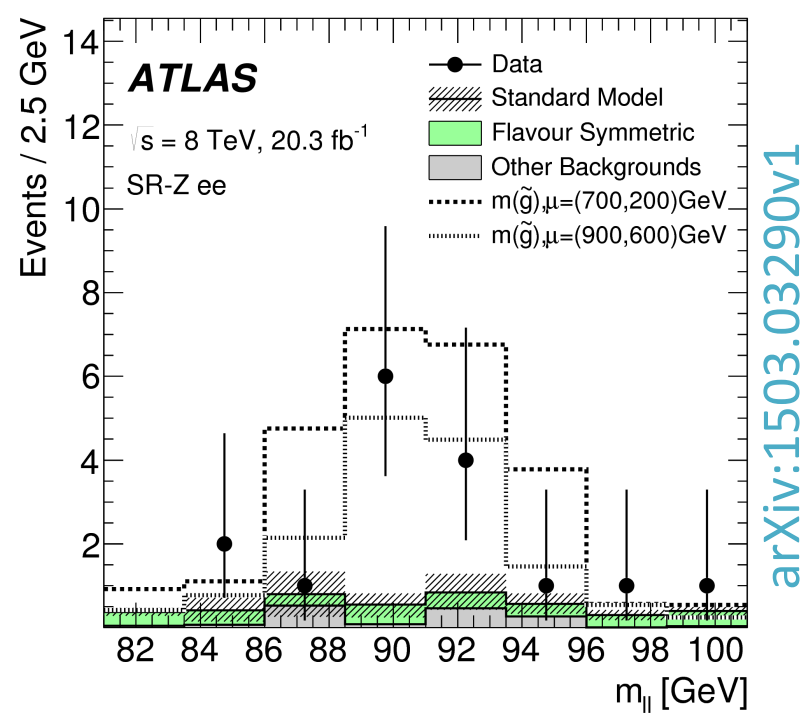

\section{$3.0 \sigma$ for ee \\ $1.7 \sigma$ for $\mu \mu$}

CMS does not see an excess at $\approx \mathrm{m}(\mathrm{Z})$
- $\mathrm{CMS}$ has an excess (edge) at masses below the $\mathrm{Z}$ mass

Edge signal included in the fit to describe the data

In edge region:

- expected: $730 \pm 40$

- observed: 860

$$
\approx 2.6 \sigma
$$

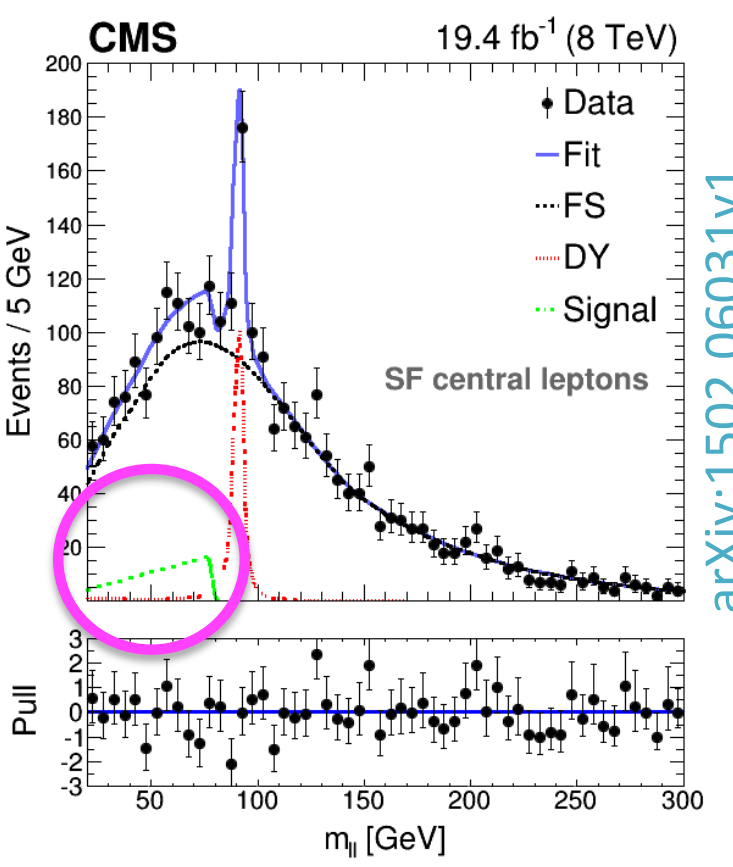

ATLAS does not see an excess in the edge

- Both experiments have excesses, but not at the same place in $\mathrm{m}_{\|}$mass ! 


\section{Higgs/Exotics Excesses (3)}

\section{ATLAS/CMS ttH}

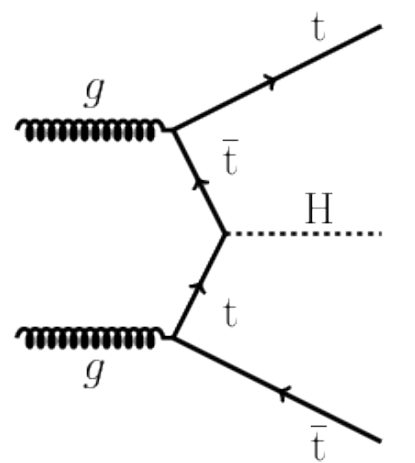

3 categories of decays :

1) $\mathrm{H} \rightarrow$ hadrons $(b b, \tau \tau)$

2) $\mathrm{H} \rightarrow$ photons

3) $\mathrm{H} \rightarrow$ leptons(from $\mathrm{WW}, \mathrm{ZZ}, \tau \tau)$

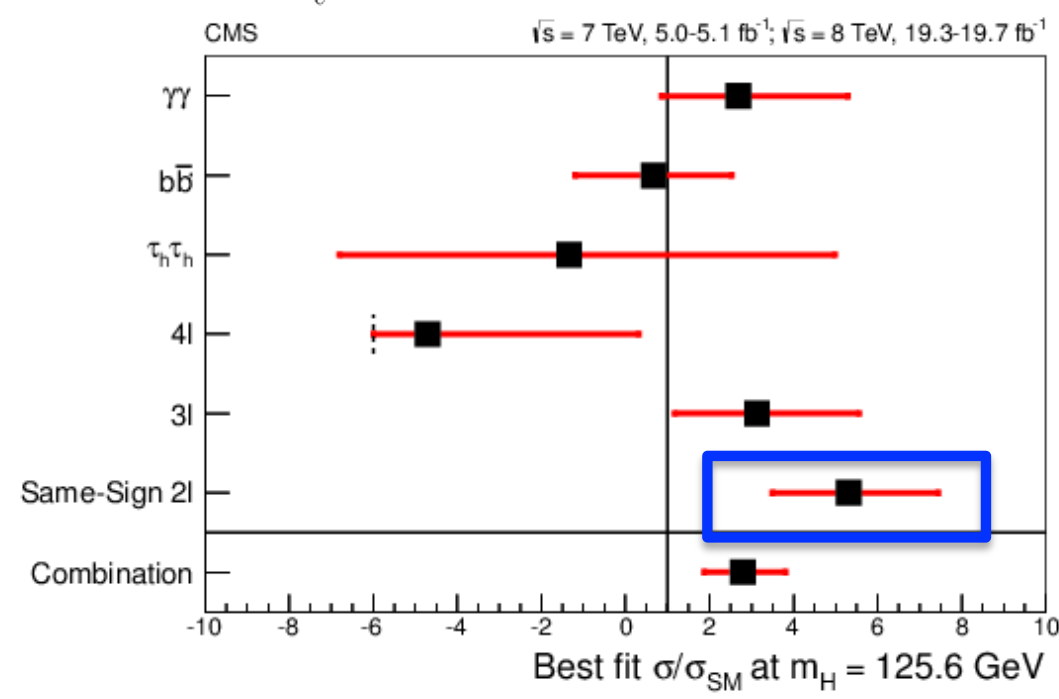

Largest discrepancy in the same-sign channel

\section{$2 \sigma$}

- An excess is also observed in ATLAS (dilepton channel). arXiv:1503.05066

\section{and ATLAS exotic same-sign+b-jets}

Sgluon pair

production :
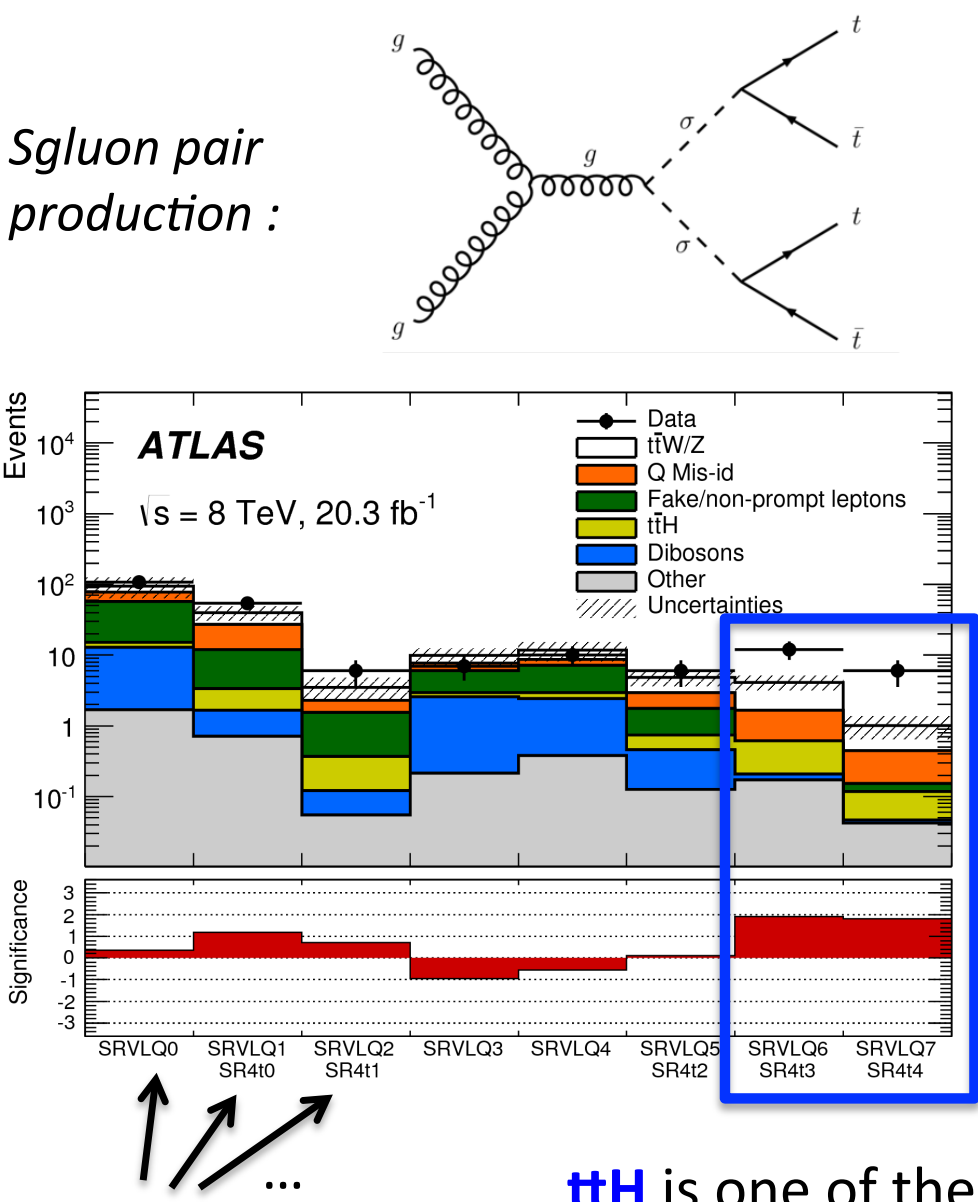

different regions designed to target different signals most dominant bkgs 


\section{Exotics Excesses (5)}
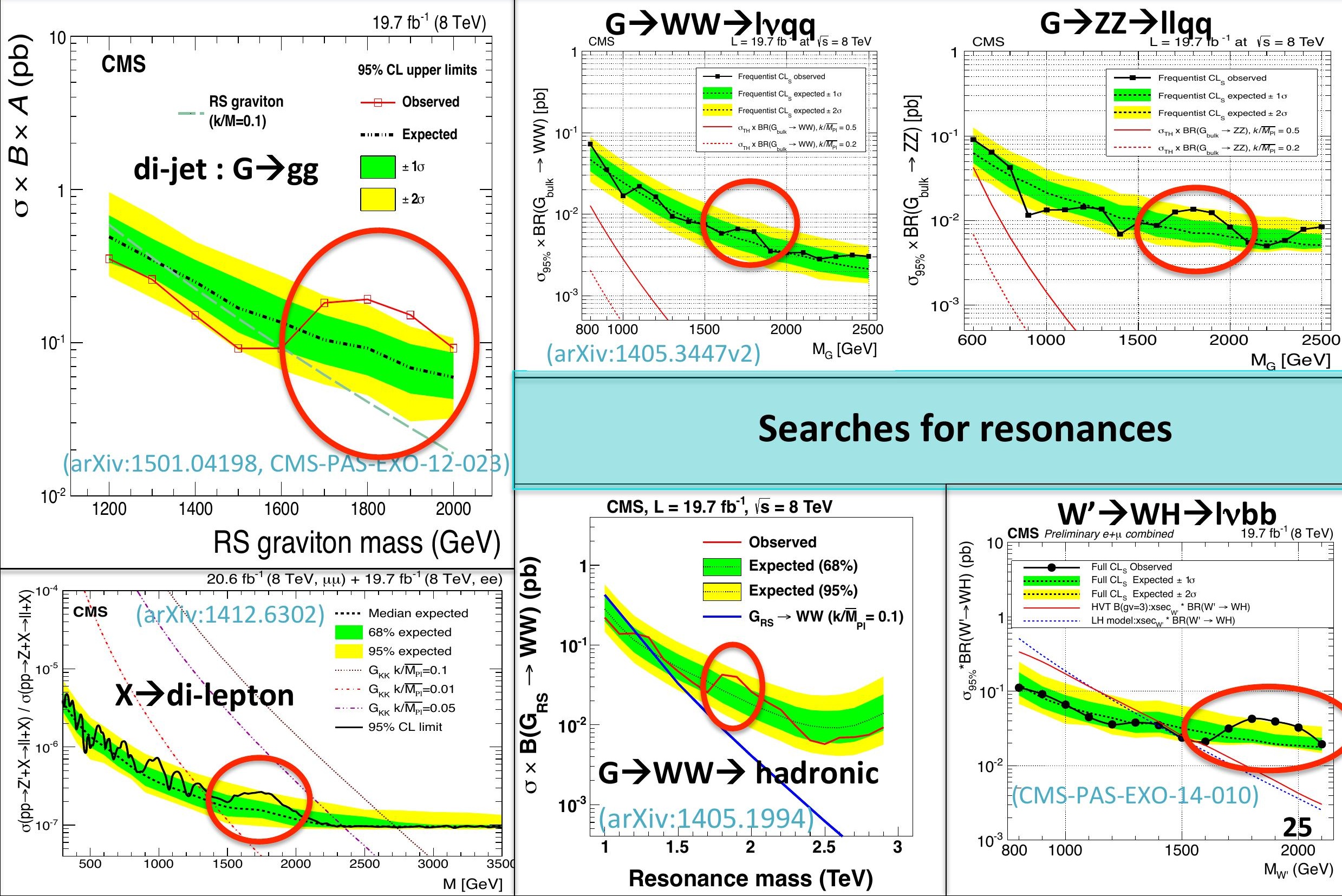

\section{Searches for resonances}
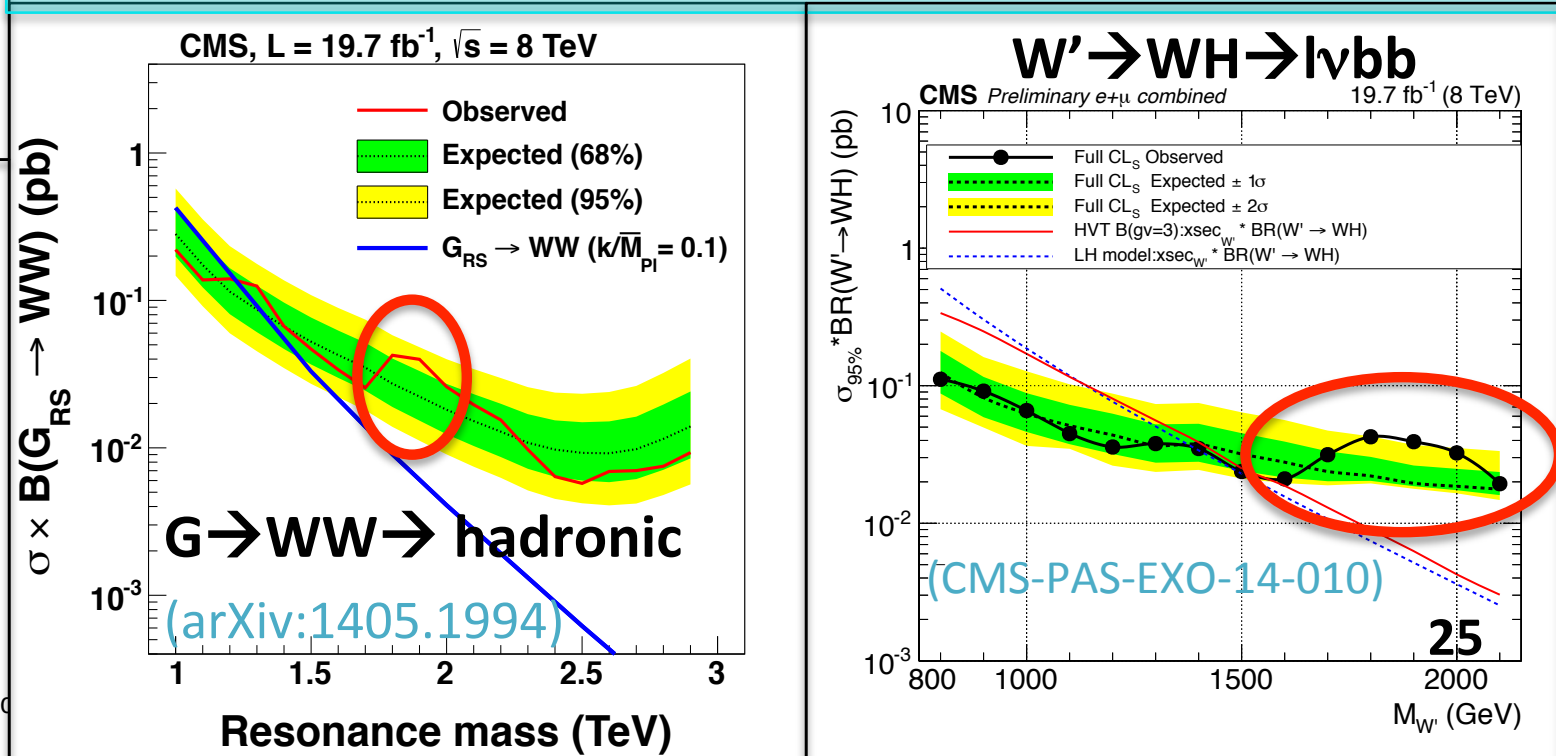


\section{Exotics Excesses (5bis)}

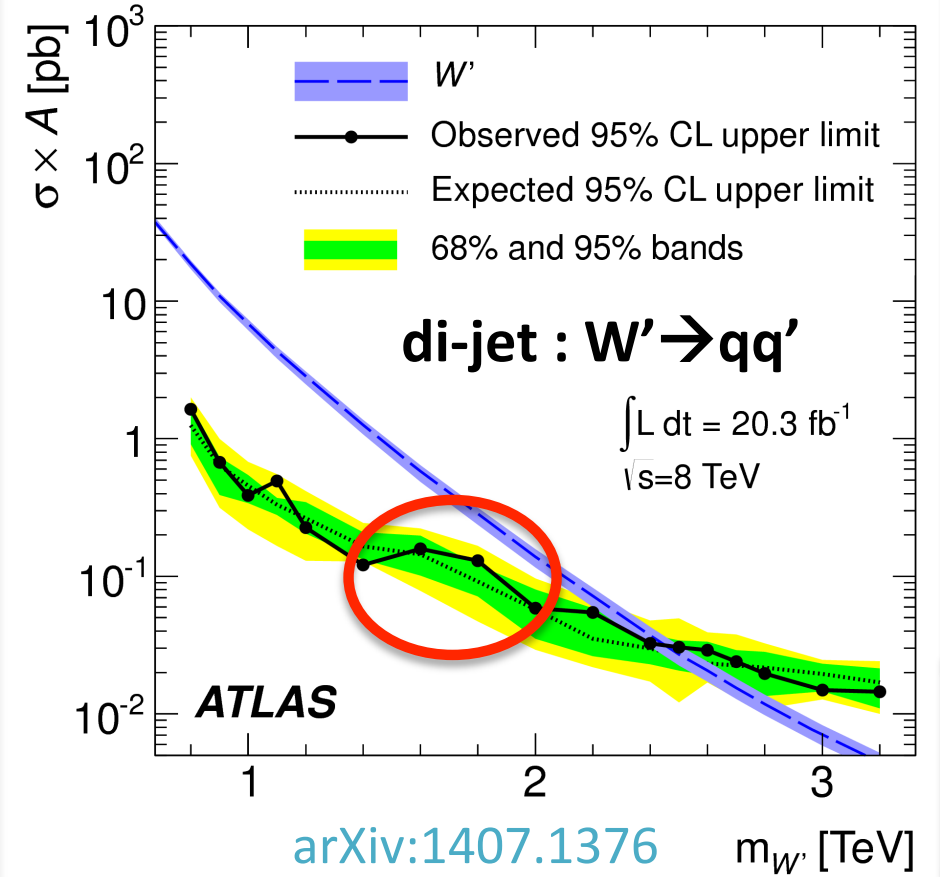

$X \rightarrow$ di-lepton

- No excess in ATLAS

(arXiv:1405.4123)
(arXiv:1503.04677)

$\mathrm{G} \rightarrow \mathrm{WW} \rightarrow \mathrm{Ivqq}$

- No excess in ATLAS
(arXiv:1409.6190)

$\mathbf{G} \rightarrow \mathrm{ZZ} \rightarrow$ Ilqq

- No excess in ATLAS

\section{Searches for resonances}

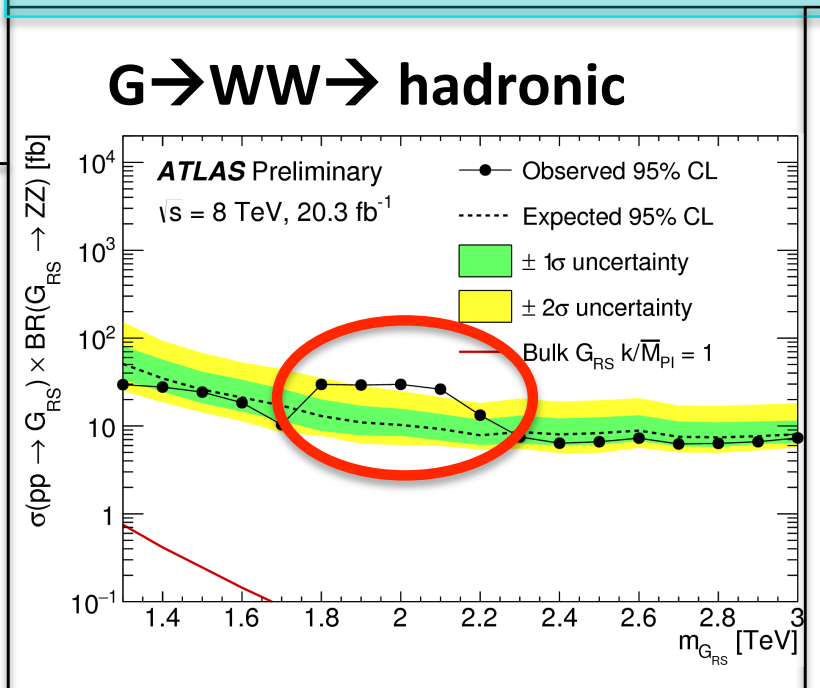

To appear soon on arXiv

\section{$\mathrm{W}^{\prime} \rightarrow \mathrm{WH} \rightarrow \mathrm{Ivbb}$}

- No excess in ATLAS, but the search stops at $\approx 1800 \mathrm{GeV}$

(arXiv:1503.08089) 


\section{LHC Run 2, ATLAS and CMS}

- 18-month LHC shutdown to consolidate and add new components

- LHC main improvements :

- centre of mass energy increased to $\sqrt{ } \mathrm{s}=13 \mathrm{TeV}$

- ATLAS main improvements :

- Muon system completion with chambers in barrel/endcap transition

- Additional innermost pixel layer (IBL) to improve tracking, vertexing and b-tagging for high pileup, and smaller radius Be beam pipe

- CMS main improvements :

- new HCAL Outer Barrel photo-detectors

- Muon system completed (fourth disk for RPC endcap region) and 72 CSC chambers added (on top of the 468 existing ones)

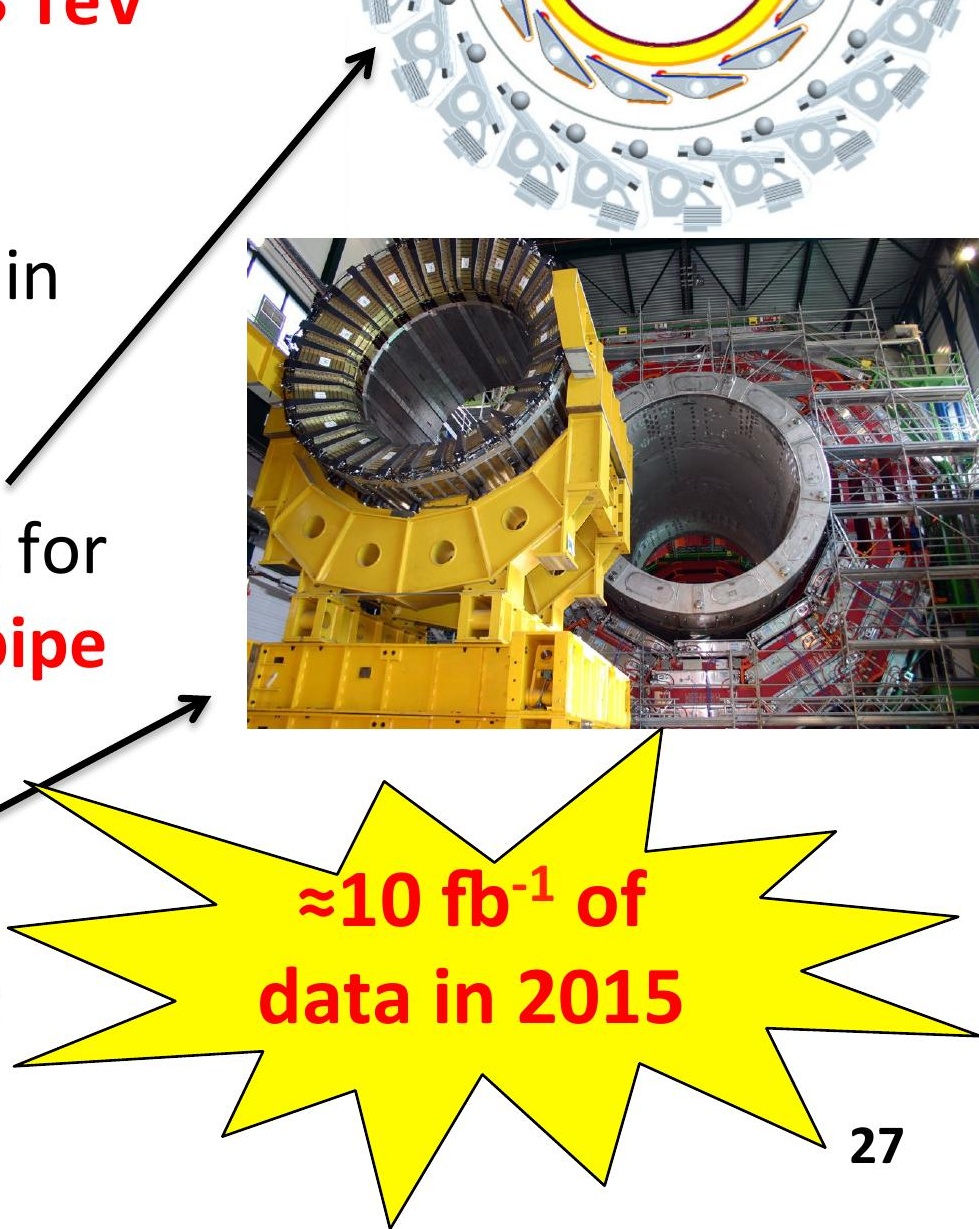




\section{Run2 (early data) : SUSY searches}

- Gluino pair production : large production increase (factor $\sim 15$ for $2 \mathrm{TeV}$ gluinos, $~ 50$ for $3 \mathrm{TeV}$ gluinos)
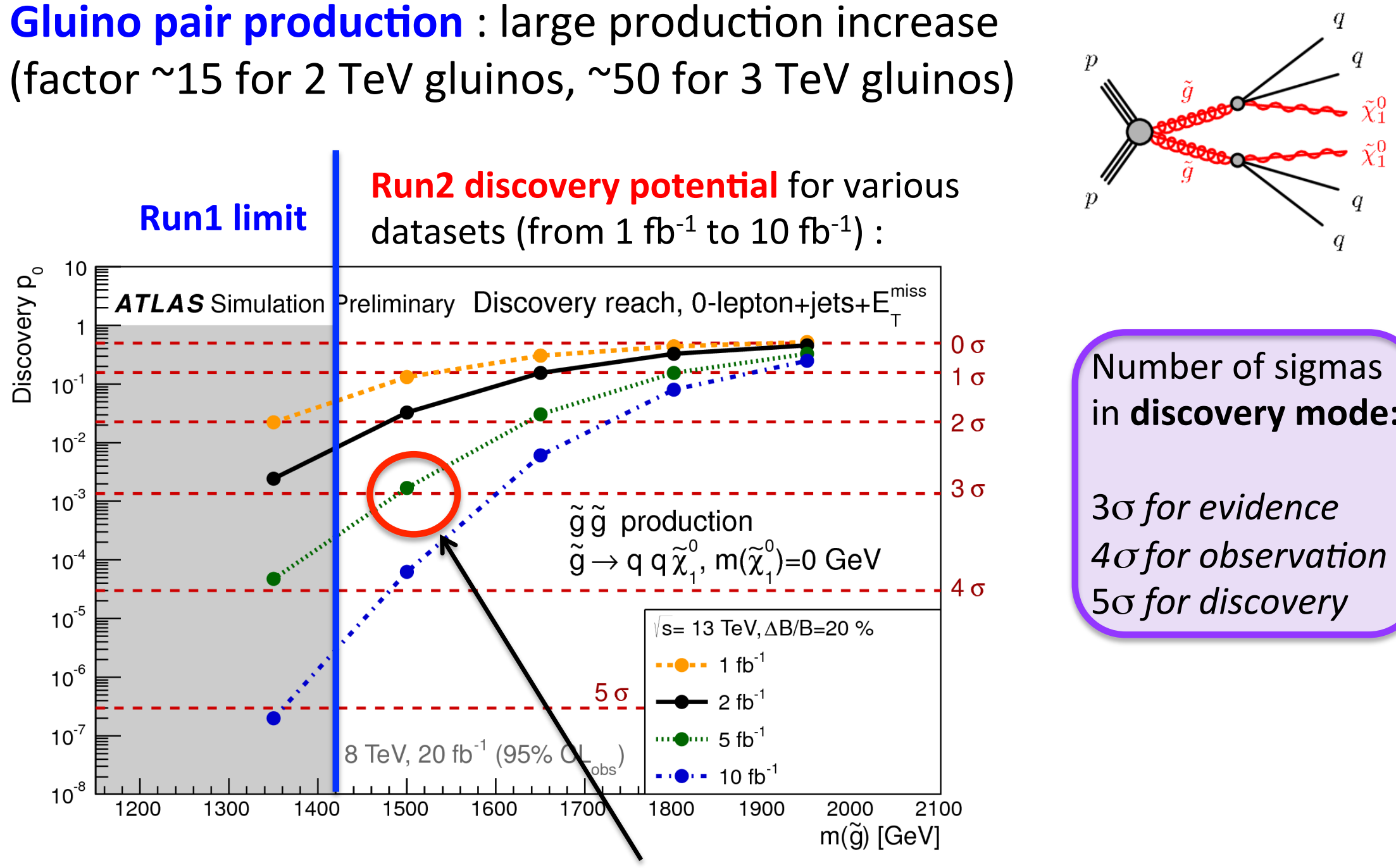

$3 \sigma$ evidence reach with $5 \mathrm{fb}^{-1}$ for gluino masses $<1500 \mathrm{GeV}$ 


\section{Run2 (early data) : Exotics searches}

- Dijet resonances search

- With $1 \mathrm{fb}^{-1}$ of $14 \mathrm{TeV}$ data, $5 \sigma$ discovery potential for :

- excited quarks up to $\approx 4 \mathrm{TeV}$

- quantum black holes up to $\approx 7 \mathrm{TeV}$

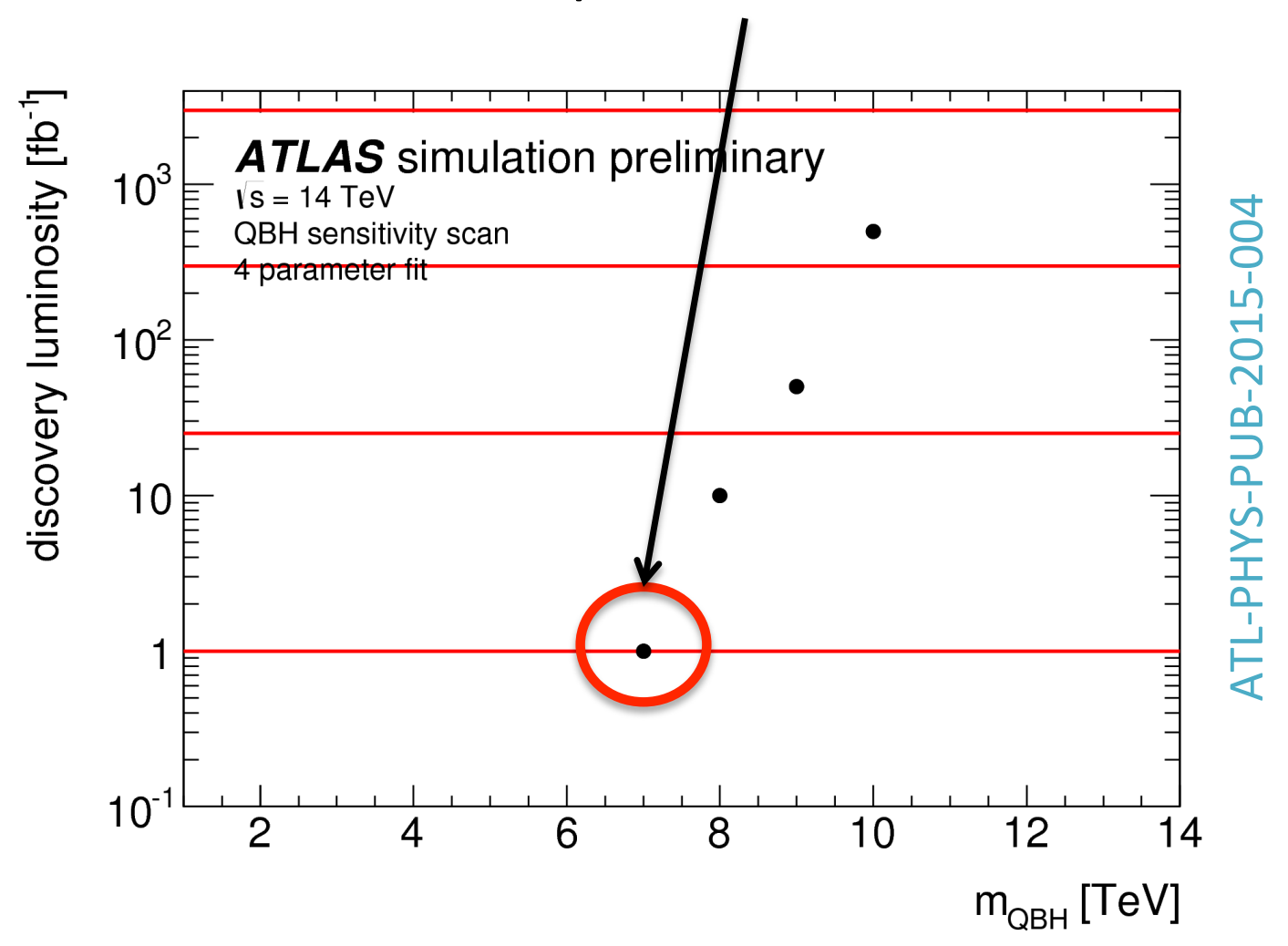




\section{Run2 (and further) : Higgs searches}

- Still quite some room left from Run1 results for BSM decays of the Higgs

- Both $\mathrm{H}^{+} \rightarrow \tau v$ and $\mathrm{A} \rightarrow \tau \tau$ will have better sensitivity with $2-5 \mathrm{fb}^{-1}$ of data

- Further into the future :

- LHC Run3 planned in 2020-2022 $\left(300 \mathrm{fb}^{-1}\right.$ of data)

- High-luminosity LHC (HL-LHC) in $\approx 2025-2035$ (3000 $\mathrm{fb}^{-1}$ of data)

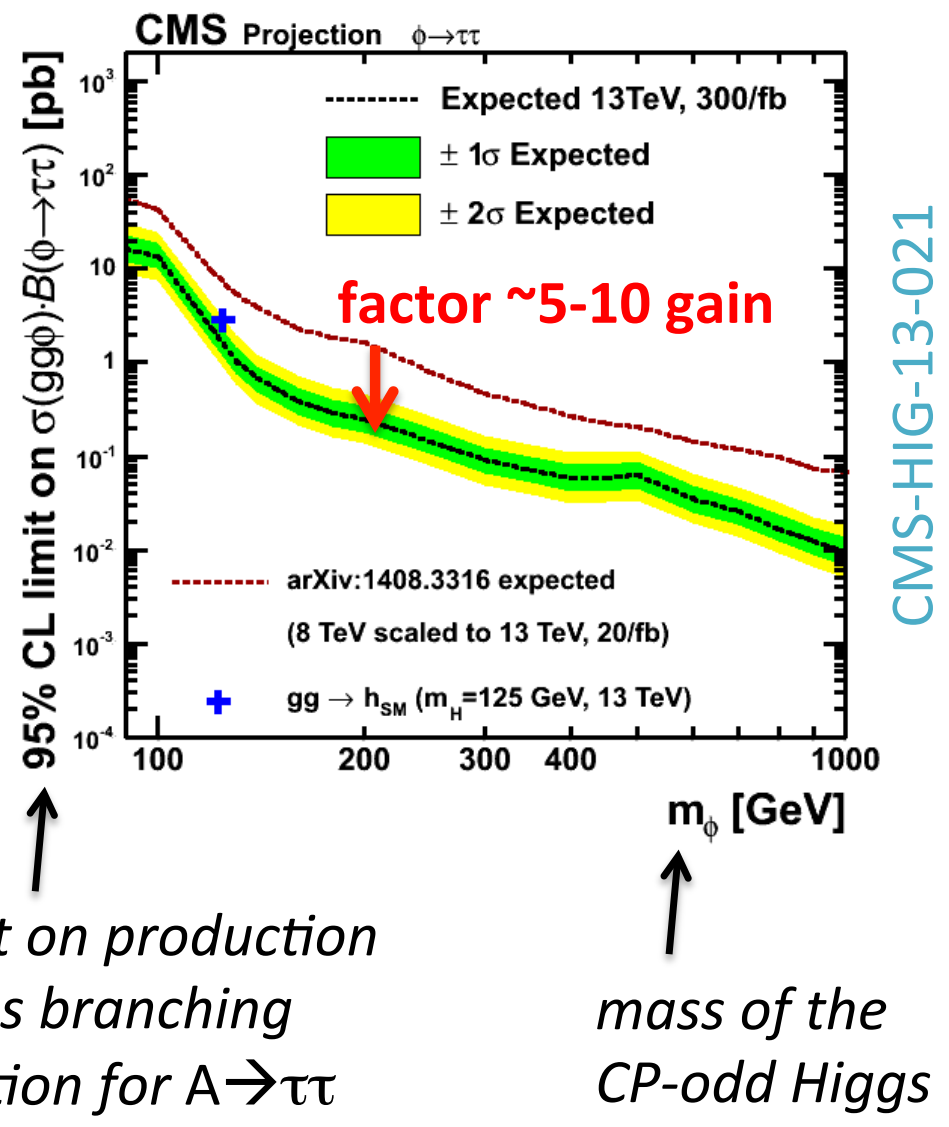




\section{Dark Matter further into the future}

Result in p.20 from $\mathrm{Zh} \rightarrow$ Il invisible:

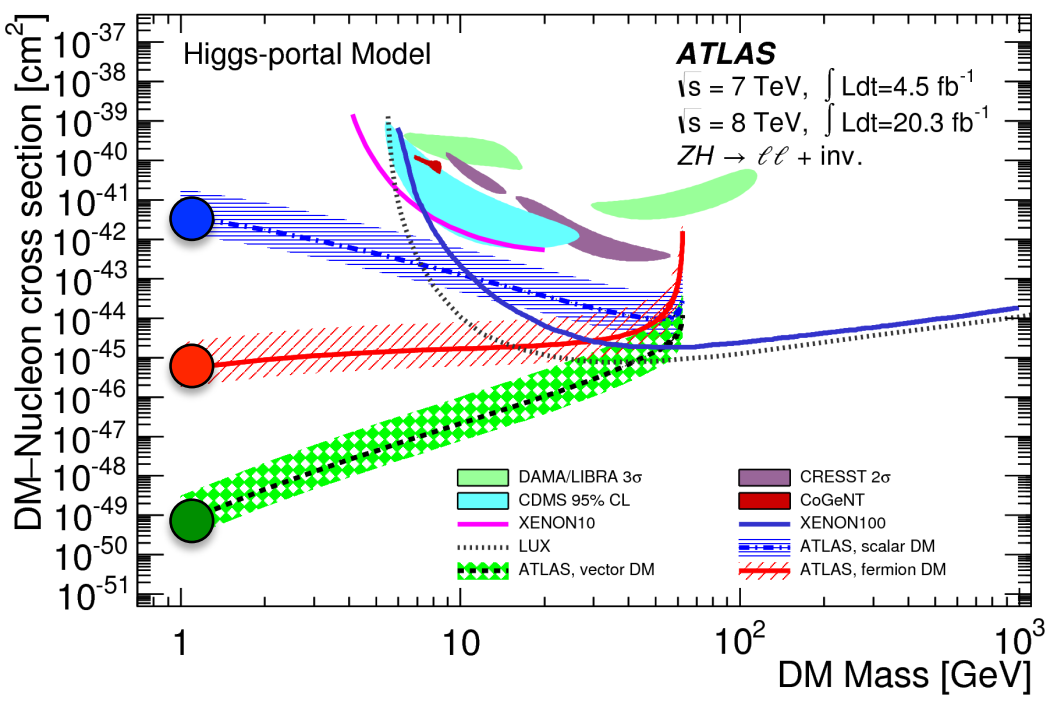

HL-LHC expectations :

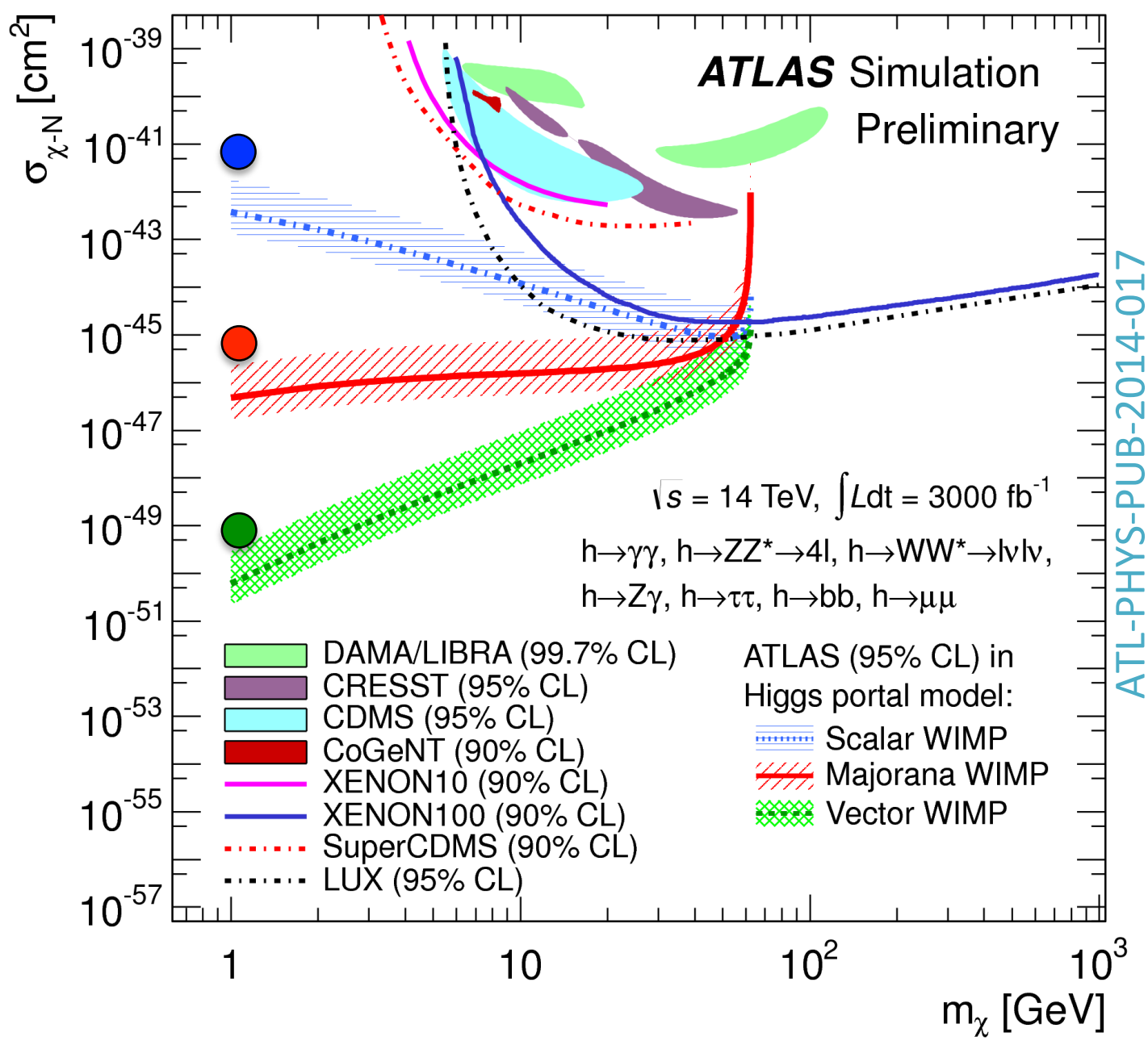

One order of magnitude gain in DM-nucleon cross section at $m_{\chi}=1 \mathrm{GeV}$ 


\section{Alternatives : Intensity Frontier}

- SHiP fixed target experiment at the SPS ?

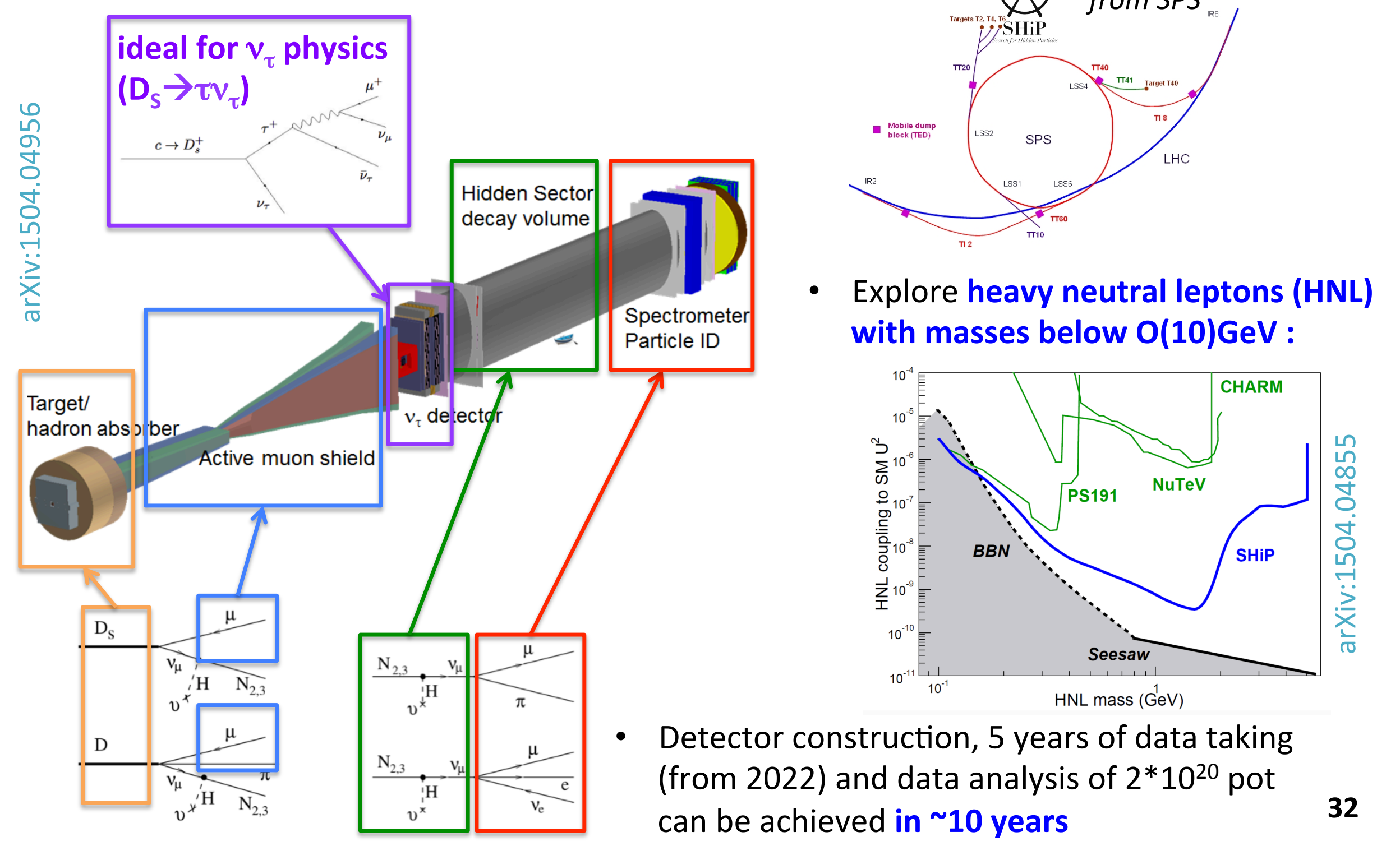




\section{Conclusions}

- New physics searched for in a lot of places during Run1

- Not possible to highlight all of them today

- The Higgs boson seems to be the one predicted by the SM

- Still room for new decays, new Higgses,...

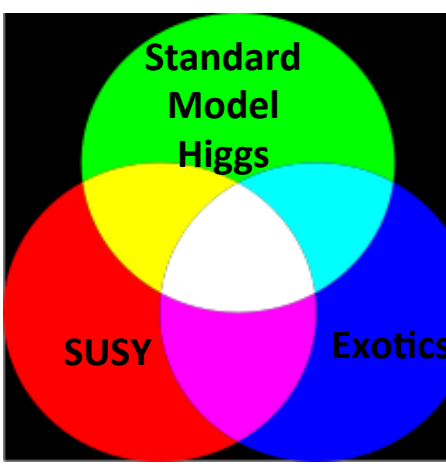

- "Natural" SUSY has suffered a lot with Run1, split-SUSY is now gaining more attention for Run2

- Analogy to EWSB model (by theorist N. Craig) ? minimal SU(2)xU(1) structure of the Glashow model (1961) is correct, but the model was missing a scalar field (Higgs) for EWSB (Weinberg in 1967) $\rightarrow$ non-minimal realization in nature

- A few excesses remaining, some of them reported by both ATLAS/CMS !

- pp collisions at $13 \mathrm{TeV}$ of Run2 will happen very shortly.

- confirm/rule out the Run1 excesses

- Explore a new window in energy with the 2015 dataset, sensitivities will increase significantly in particular in high mass regions ! 
Back-Up Slides 


\section{LHC, ATLAS and CMS}




\title{
The LHC Program
}

\section{LHC goal for 2015 and for Run 2 and 3}

\author{
Integrated luminosity goal: \\ $2015: 10 \mathrm{fb}^{-1}$ \\ Run2: $\sim 100-120 \mathrm{fb}^{-1}$
}

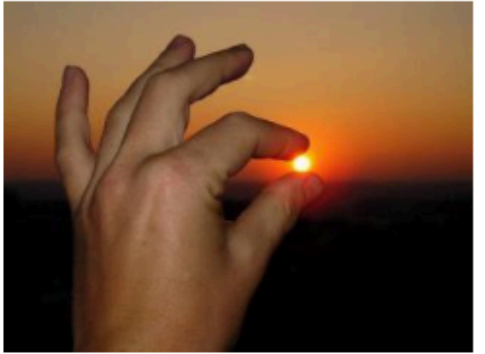

(better estimation by end of 2015)

\section{$300 \mathrm{fb}^{-1}$ before LS3}

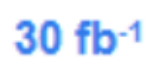

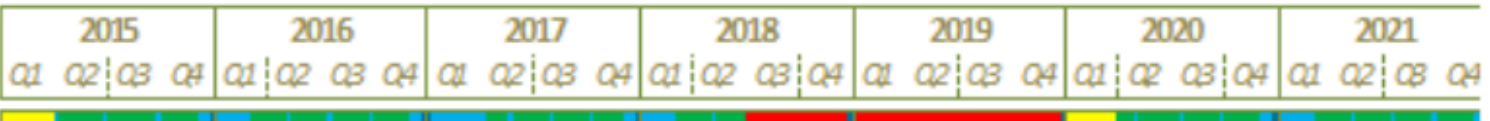

$$
\begin{aligned}
& \text { IHC } \\
& \text { Injectors }
\end{aligned}
$$
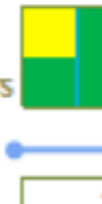

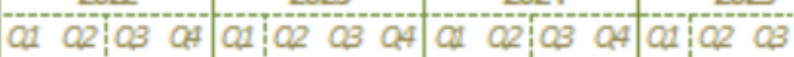

\begin{tabular}{|l|l|l|l|l|}
\hline YETS & & LS 3 & \\
\hline
\end{tabular}




\section{ATLAS and CMS : More details}

\section{- The main differences between the ATLAS and CMS detectors are:}

\section{ATLAS}

2T solenoid (Inner Tracker inside, HCAL outside of B-field) + toroid: 0.5T (barrel), 1T (endcap)

$\rightarrow$ good for jet resolution, worse for $\mathrm{e} / \gamma$

Tracker

B-field

Si pixels and strips + transition radiation tracker

$\rightarrow$ high resolution, granularity, "continuous"

tracking at large radii

$\sigma / p_{\mathrm{T}} \sim 5 \times 10^{-4} p_{\mathrm{T}}+0.01$

$\pi$ with $\mathrm{pT}=1 \mathrm{GeV}$

e with $\mathrm{pT}=5 \mathrm{GeV}$

$84 \%$ reco efficiency (material budget, B-field)

$90 \%$ reco efficiency

Liquid argon $+\mathrm{Pb}$ absorbers $\rightarrow$ high granularity

$\sigma / E \sim 10 \% / V E+0.007$

$1.0-1.5 \%$ E resolution

$1.3-2.3 \%$ E resolution

$\mathrm{Fe}+$ scintillator / Cu+Lar (10ג)

$\sigma / \mathrm{E} \sim 50 \% / \mathrm{VE}+0.03 \mathrm{GeV}$

$2 \%$

$20 \mathrm{GeV}$

$\sigma / p_{\mathrm{T}} \sim 2 \%$ at $50 \mathrm{GeV}$ to $10 \%$ at $1 \mathrm{TeV}$

(Inner Tracker + muon system)

\section{CMS}

4T solenoid + return yoke

(ECAL and part of HCAL inside)

$\rightarrow$ good for e/ $\gamma$ resolution, worse for jet

Si pixels and strips (fully Silicon)

$\rightarrow$ high resolution, granularity

$\sigma / p_{\mathrm{T}} \sim 1.5 \times 10^{-4} p_{\mathrm{T}}+0.005$

$80 \%$ reco efficiency

$85 \%$ reco efficiency

$\mathrm{PbWO}_{4}$ crystals $\rightarrow$ high resolution

$\sigma / E \sim 3 \% / V E+0.003$

$0.8 \% \mathrm{E}$ resolution

$2.0 \%$ E resolution

Brass + scintillator $(7 \lambda+$ catcher $)$

$\sigma / \mathrm{E} \sim 100 \% / \mathrm{VE}+0.05 \mathrm{GeV}$

$5 \%$

$40 \mathrm{GeV}$

$\sigma / p_{\mathrm{T}} \sim 1 \%$ at $50 \mathrm{GeV}$ to $10 \%$ at $1 \mathrm{TeV}$

(Inner Tracker + muon system) 


\section{The CMS Detector}

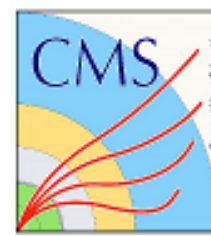

CMS Detector

Weight $\quad: 14,000$ tonnes

Diameter $\quad: 15.0 \mathrm{~m}$

Length $: 28.7 \mathrm{~m}$

Magnetic field: $3.8 \mathrm{~T}$

a

Steel

Return Yoke

Electromagnetic Calorimeter

Hadron Calorimeter

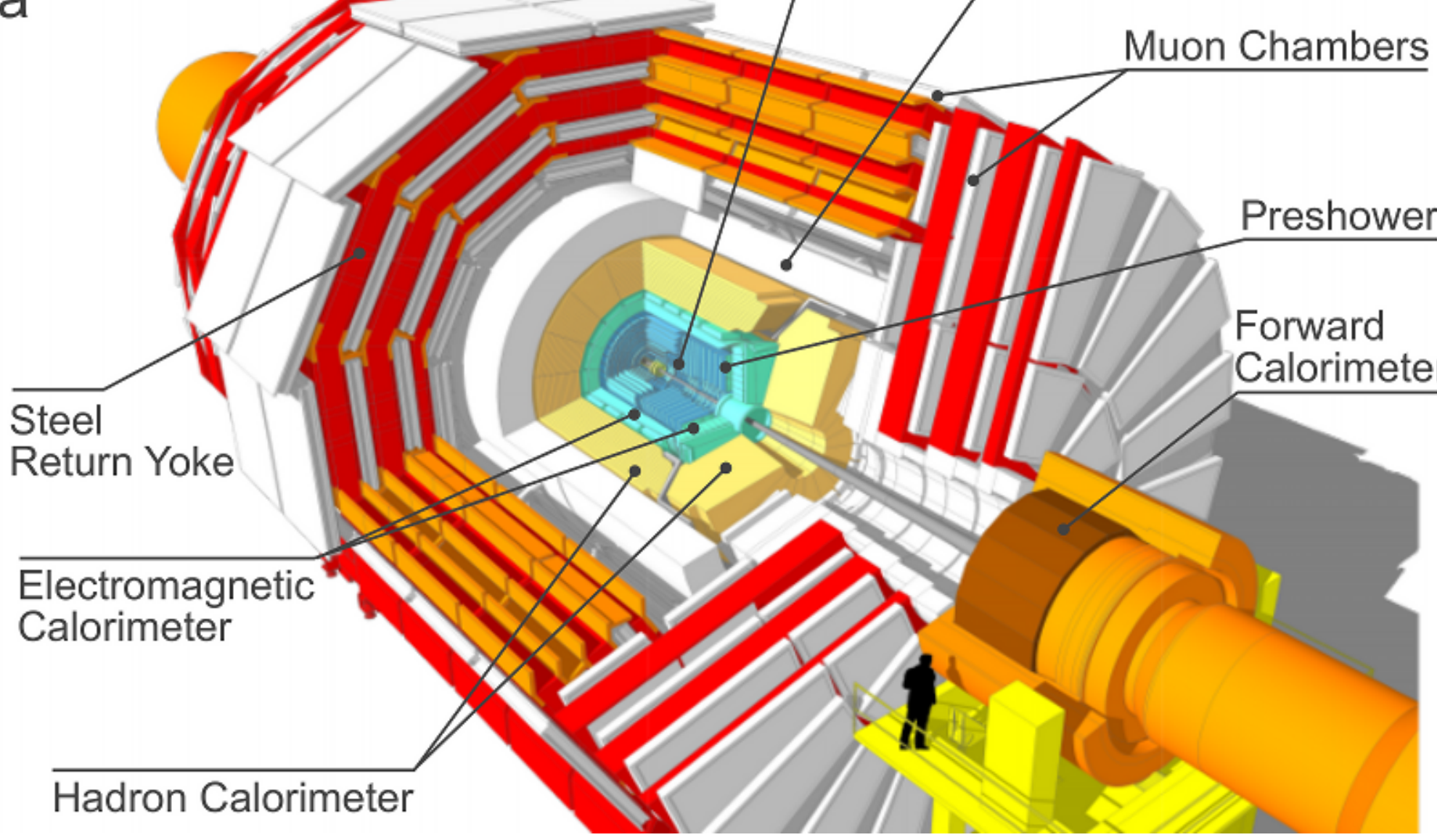

Superconducting Solenoid 


\section{Particle Physics Object Detection}

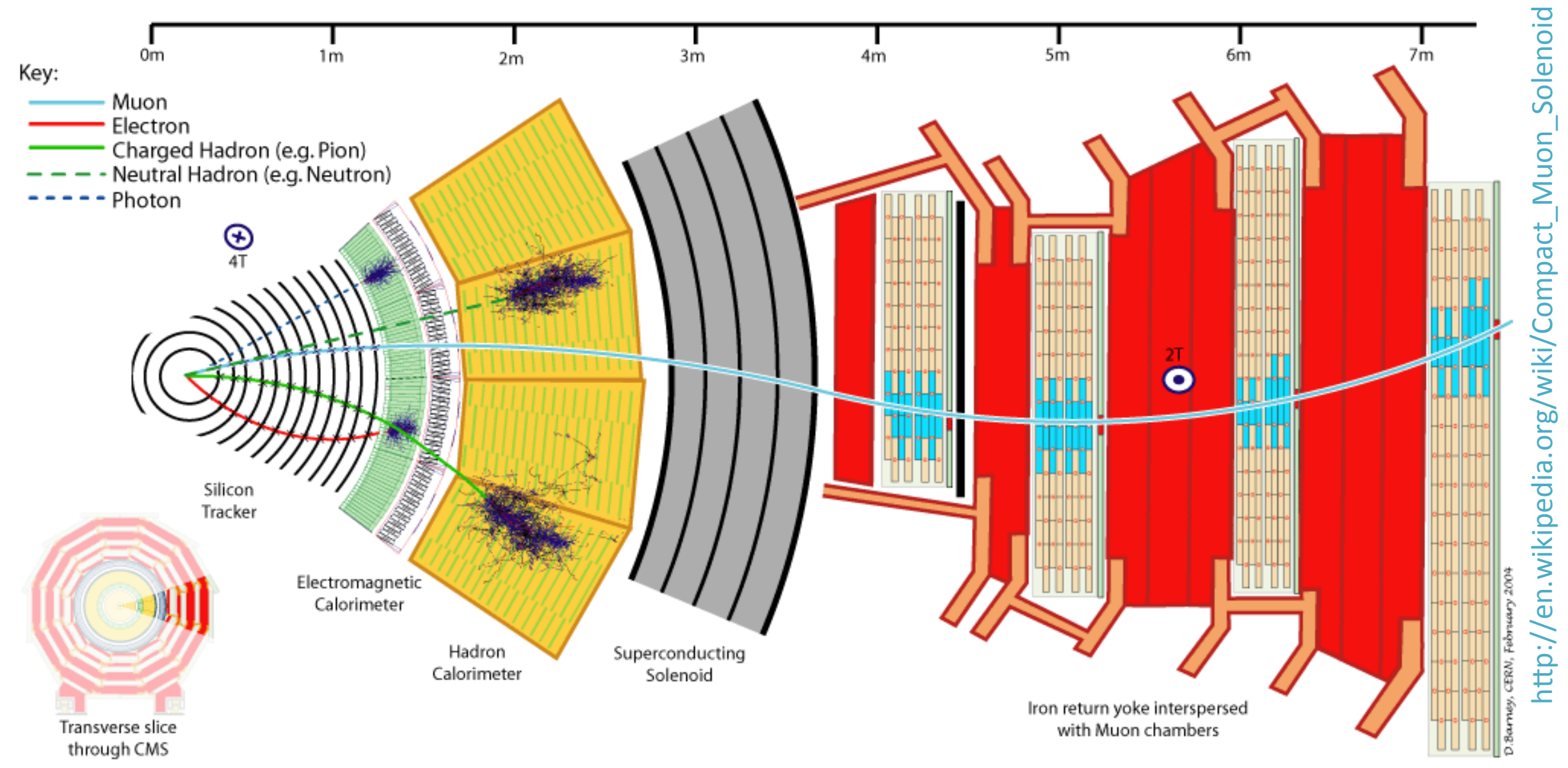




\section{Searches for SM Deviations}




\section{Run1 : Search for deviations from the SM}

- In the Standard Model, deviations were looked for in :

- Cross section measurements

- Anomalous couplings

- Resonances (ttbar,...)

- Indirect effects (B-phypics)

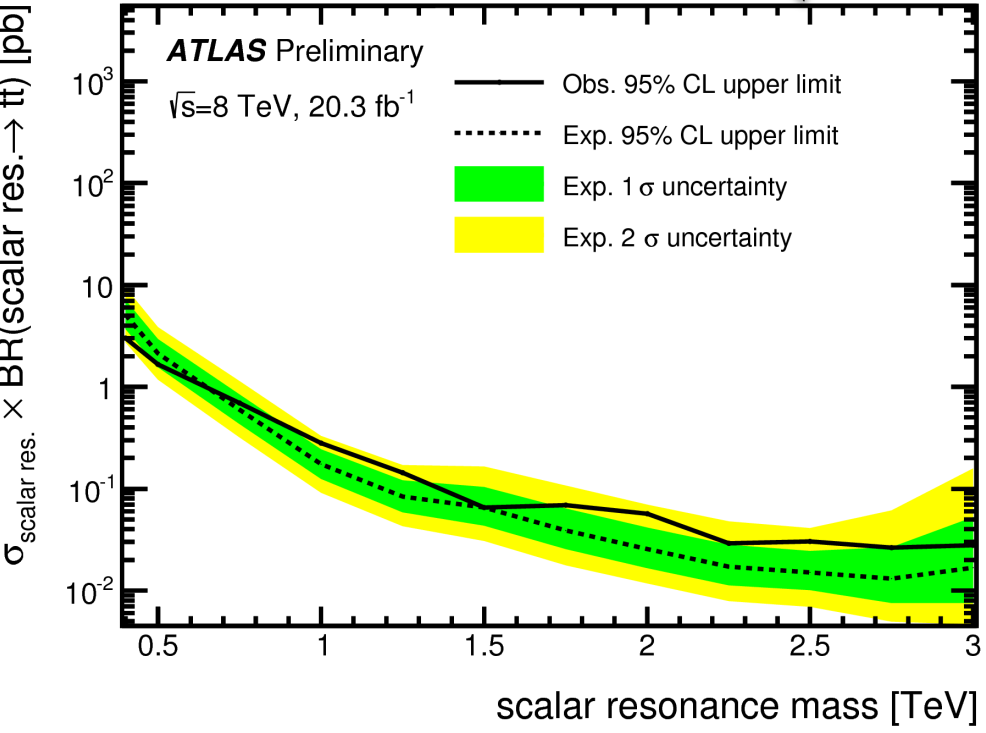

\begin{tabular}{|c|c|c|c|c|}
\hline \multirow{3}{*}{$\begin{array}{l}\text { Mar. } 2015 \\
\text { CMS measurements } \\
\text { vs. NLO (NNLO) theory }\end{array}$} & & \multicolumn{3}{|c|}{ CMS Preliminary } \\
\hline & & \multirow{2}{*}{\multicolumn{2}{|c|}{$\begin{array}{l}7 \mathrm{TeV} \text { CMS measurement (stat,stat+sys) } \\
8 \mathrm{TeV} \text { CMS measurement (stat,stat+sys) }\end{array}$}} & $\ldots, \ldots$ \\
\hline & & & & 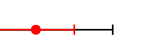 \\
\hline$\gamma \gamma$, (NNLO th.) & $\longmapsto$ & 一 & $1.06 \pm 0.01 \pm 0.12$ & $5.0 \mathrm{fb}^{-1}$ \\
\hline $\mathbf{W} \gamma$ & - & & $1.16 \pm 0.03 \pm 0.13$ & $5.0 \mathrm{fb}^{-1}$ \\
\hline$Z_{\gamma}$ & $\longmapsto+1$ & & $0.98 \pm 0.01 \pm 0.05$ & $5.0 \mathrm{fb}^{-1}$ \\
\hline$Z_{\gamma}$ & 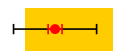 & & $0.98 \pm 0.01 \pm 0.05$ & $19.5 \mathrm{fb}^{-1}$ \\
\hline$W W+W Z$ & -2 & $\longrightarrow$ & $1.05 \pm 0.13 \pm 0.15$ & $4.9 \mathrm{fb}^{-1}$ \\
\hline WW & $\longmapsto$ & $\longrightarrow$ & $1.11 \pm 0.04 \pm 0.10$ & $4.9 \mathrm{fb}^{-1}$ \\
\hline WW, (NNLO th.) & —10. & & $1.01 \pm 0.02 \pm 0.08$ & $19.4 \mathrm{fb}^{-1}$ \\
\hline VVL & & & $1.17 \pm 0.07 \pm 0.07$ & $4.9 \mathrm{TO}^{-}$ \\
\hline WZ & & $\longrightarrow$ & $1.12 \pm 0.03 \pm 0.07$ & $19.6 \mathrm{fb}^{-1}$ \\
\hline ZZ & 0 & & $0.99 \pm 0.14 \pm 0.07$ & $4.9 \mathrm{fb}^{-1}$ \\
\hline ZZ & $+\ldots$ & & $1.00 \pm 0.06 \pm 0.08$ & $19.6 \mathrm{fb}^{-1}$ \\
\hline t: & $\frac{1}{1}$ & & ${ }^{1.5}$ Section & $\sigma_{\exp } /$ \\
\hline
\end{tabular}

- WW production cross section that was puzzling during Run1

- NNLO theory calculations could explain the discrepancy observed 


\section{Run1 : Standard Model results}

\section{Anomalous couplings :}

Quartic couplings in the SM :

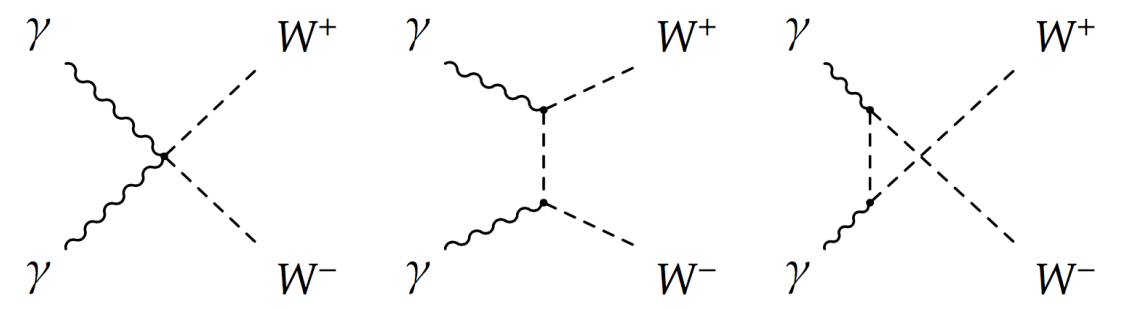

- Add anomalous quartic couplings via effective Lagrangian (independent of the SM ones)

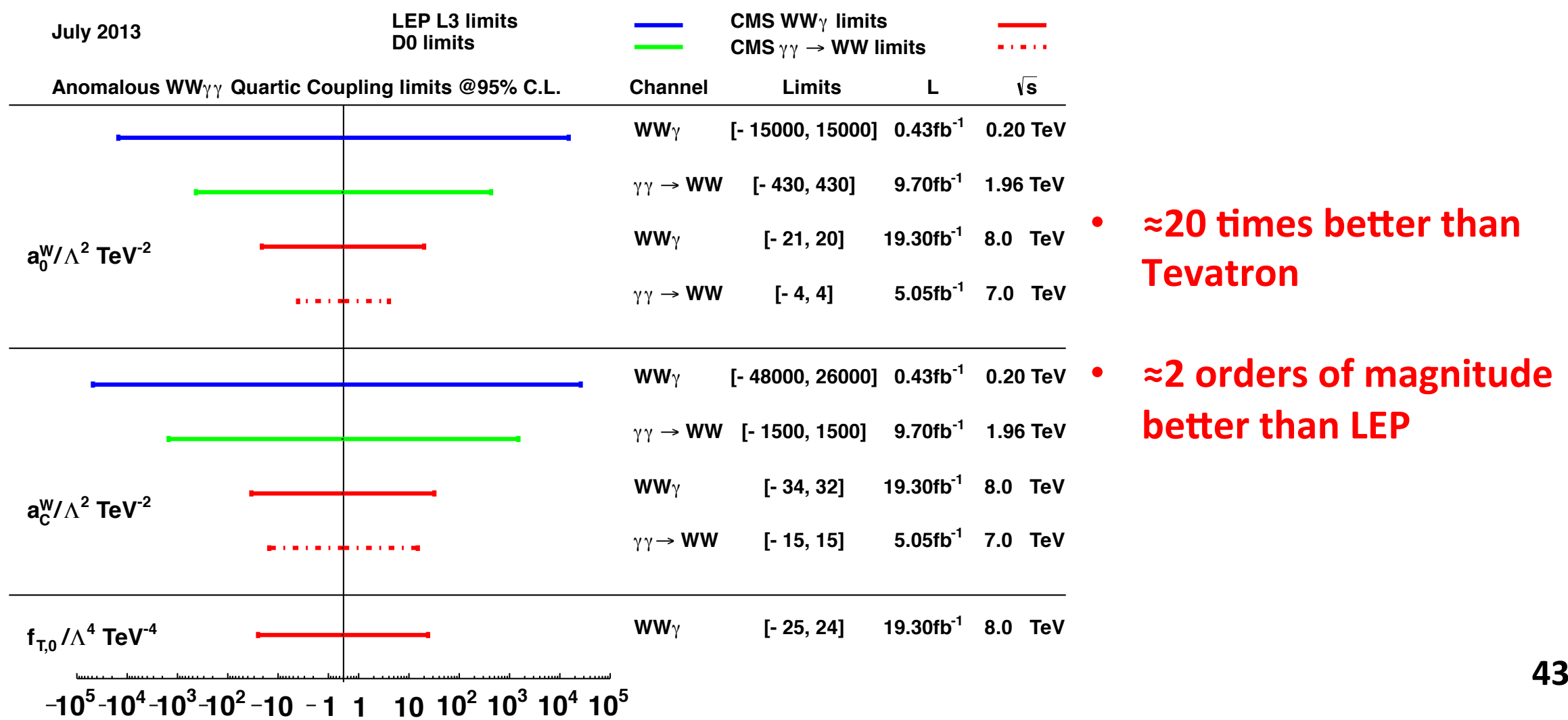




\section{More material on mono-object searches}




\section{Dark Matter Searches at LHC (3)}

\section{DM annihilation rate :}

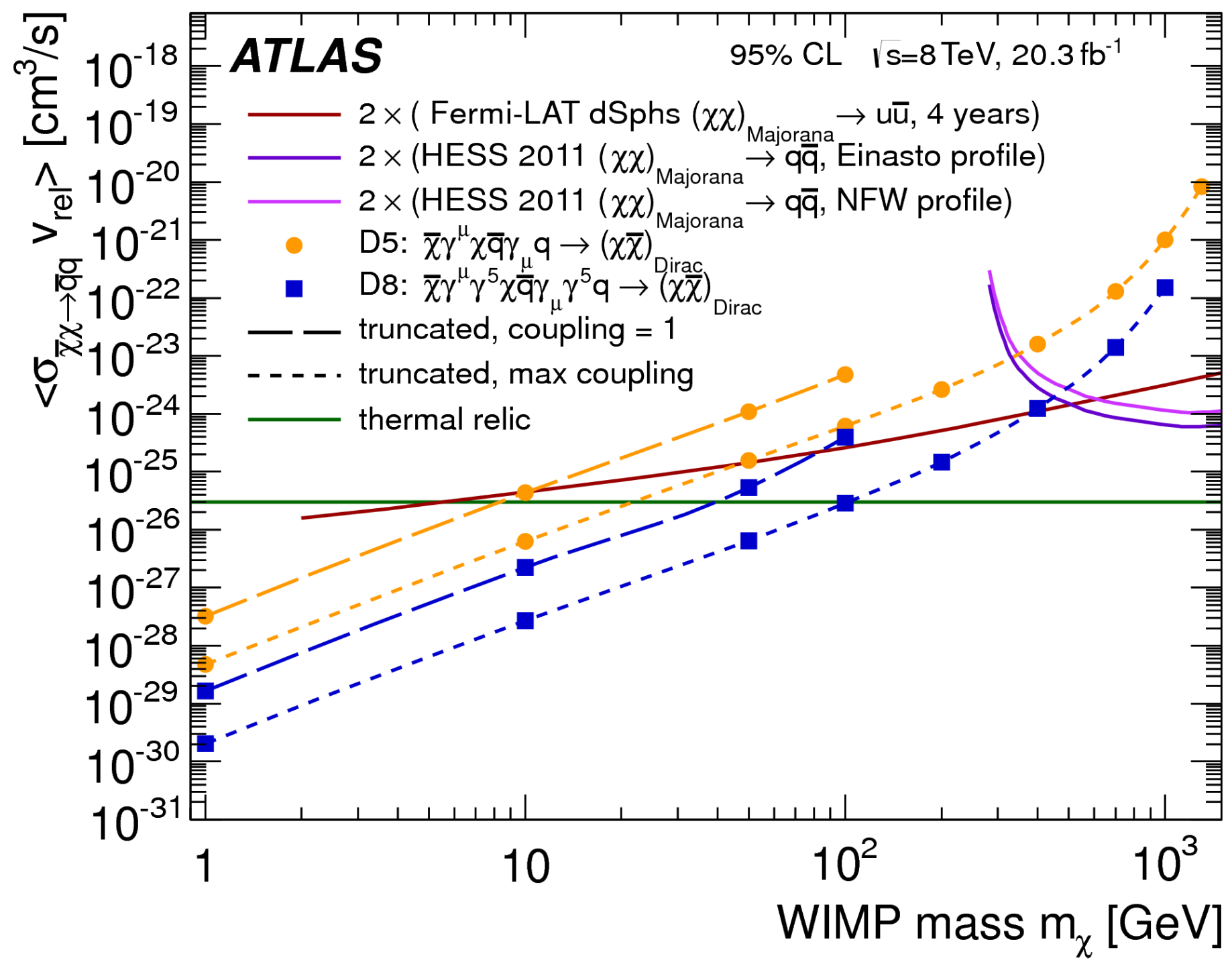




\section{Mono-photon : Limits on SUSY}

\section{Limit on SUSY compressed result (from energetic photon)}

$\sigma\left(p p \rightarrow \tilde{q} \tilde{q}^{*} \gamma+X\right)$
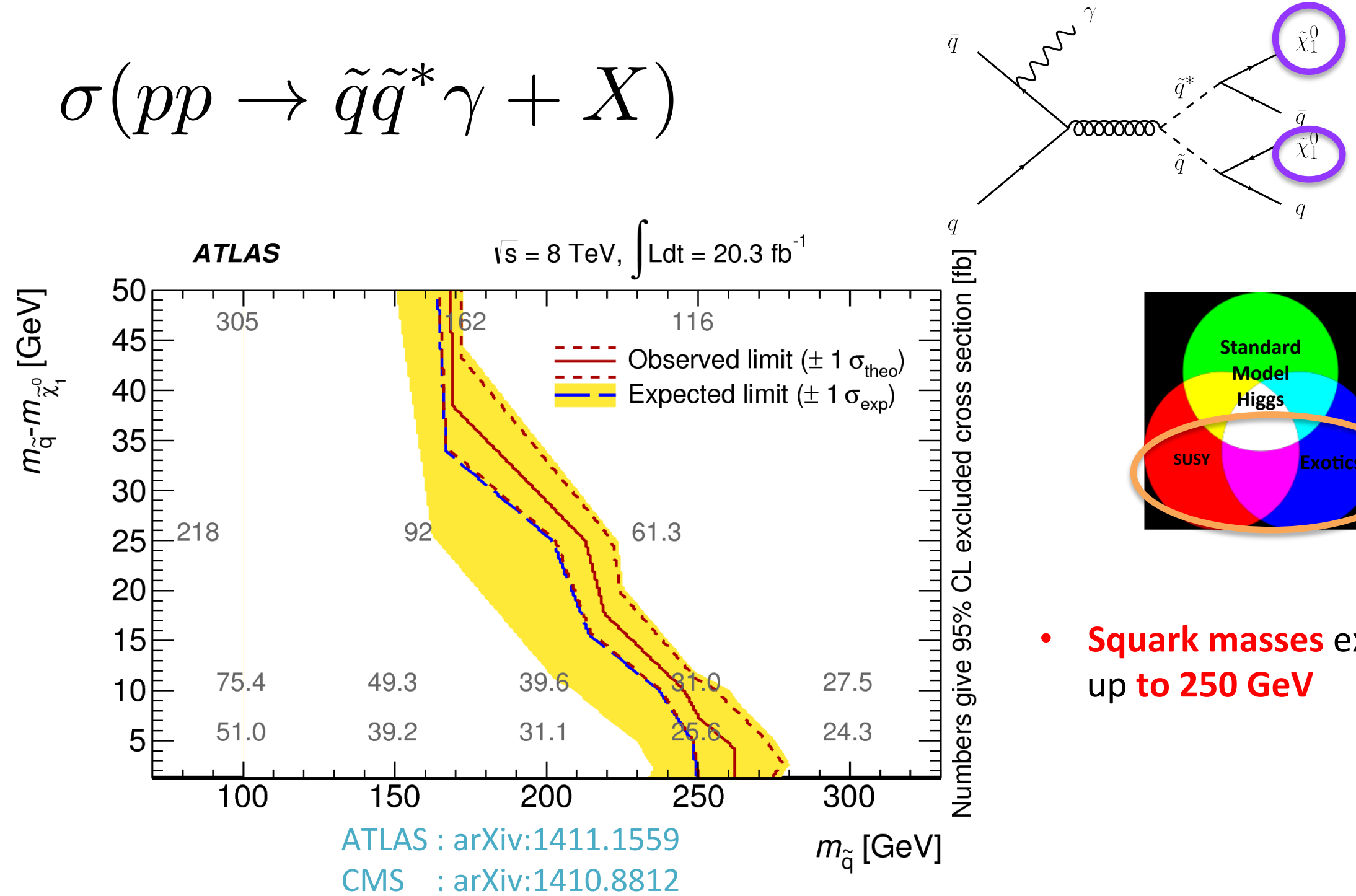

운

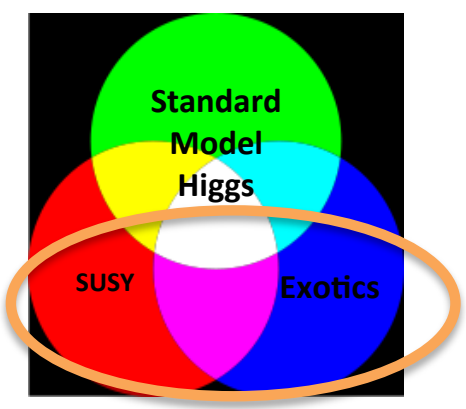

- Squark masses excluded up to $250 \mathrm{GeV}$ 


\section{General search}




\section{Run1 : Model-Independent General Search}

- 697 final states put together

- Compare data to backgrounds

- Number of events

- Distributions, like missing energy

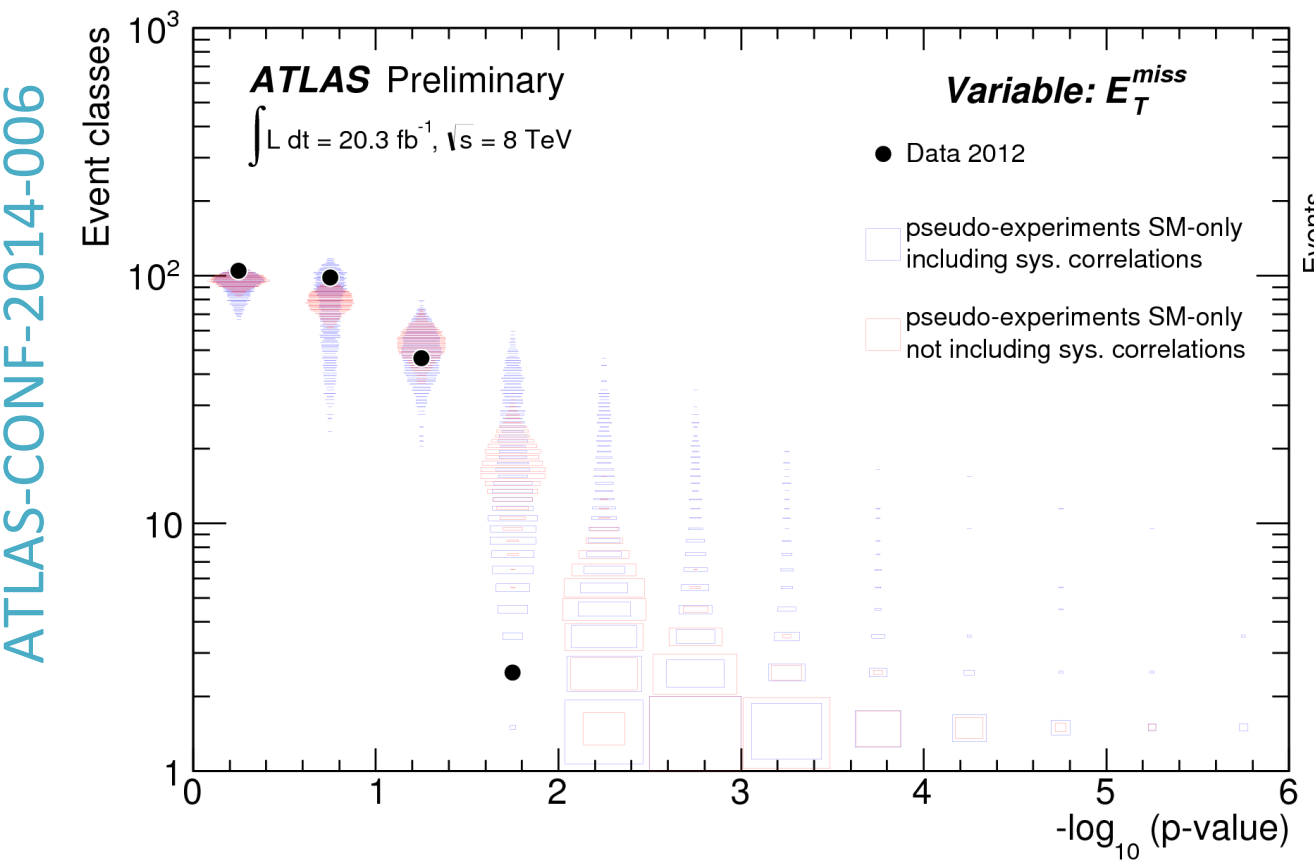

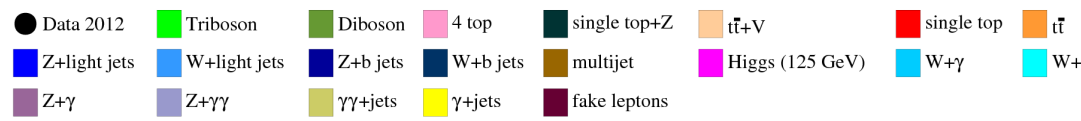
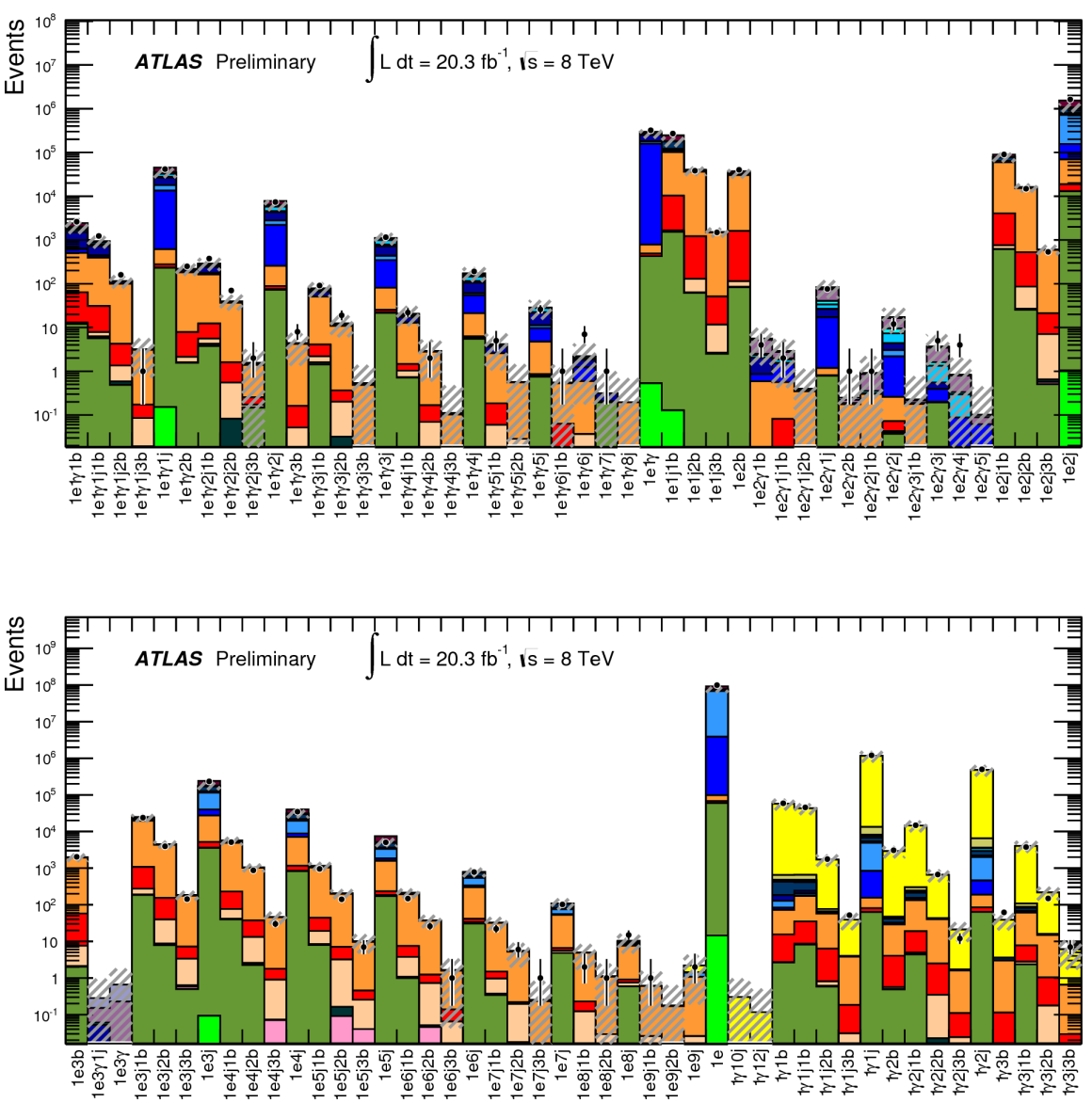

No significant deviation found 


\section{More material on excesses}


2 leptons (on/off-Z) + jets + missing energy (ATLAS)

2 leptons (on/off-Z) + jets + missing energy

Background description ok everywhere
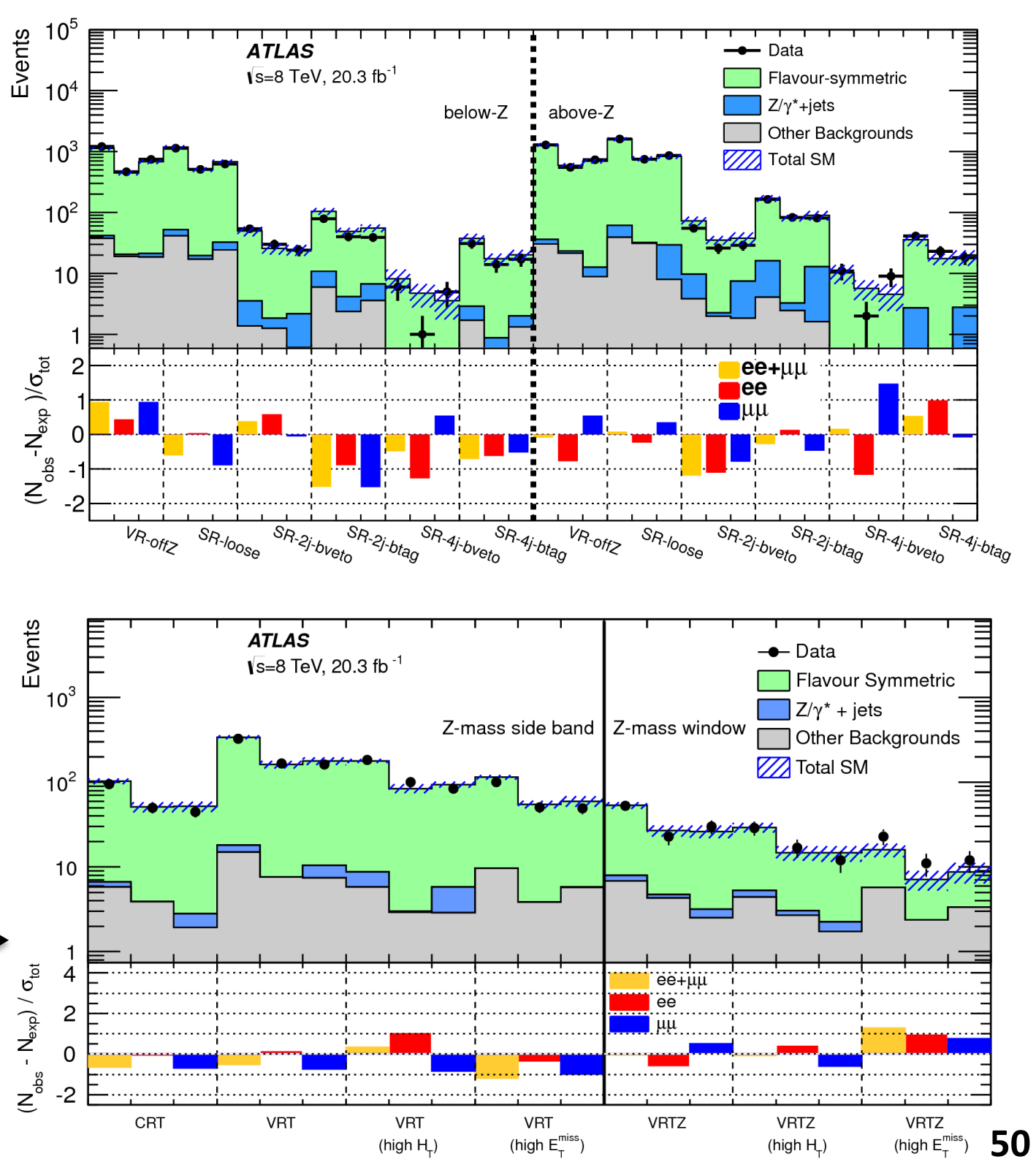


\section{CMS ttH}

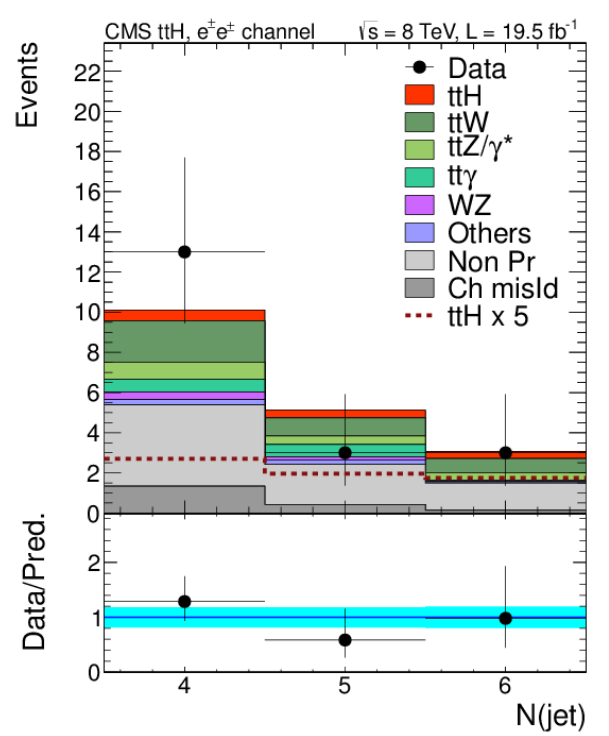

arXiv:1408.1682v2 [hep-ex]

\section{CMS ttH}
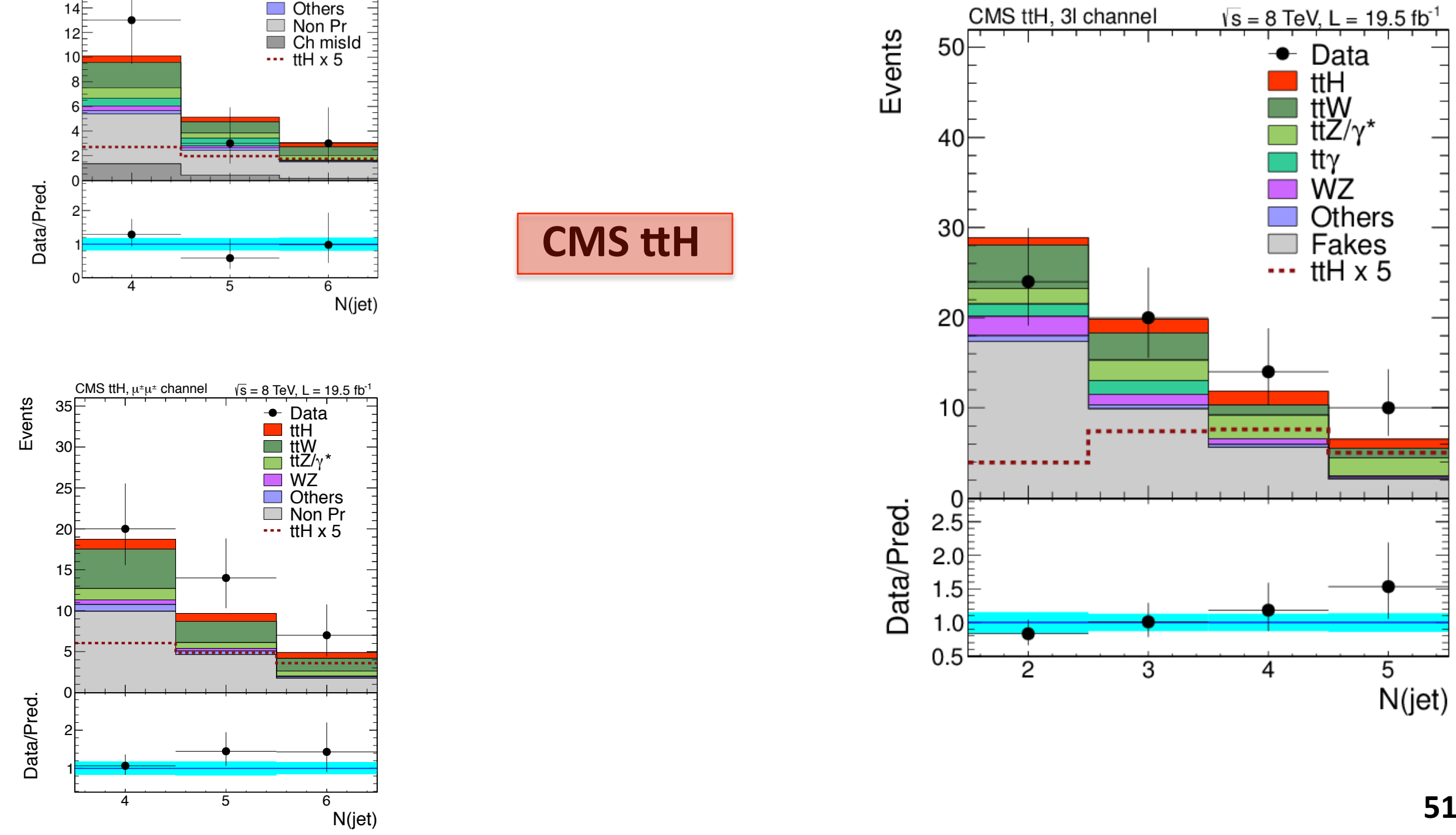


\section{Majorana Neutrinos}

\section{Heavy neutrinos and W bosons with right-handed couplings}

\section{Same-sign analysis :}

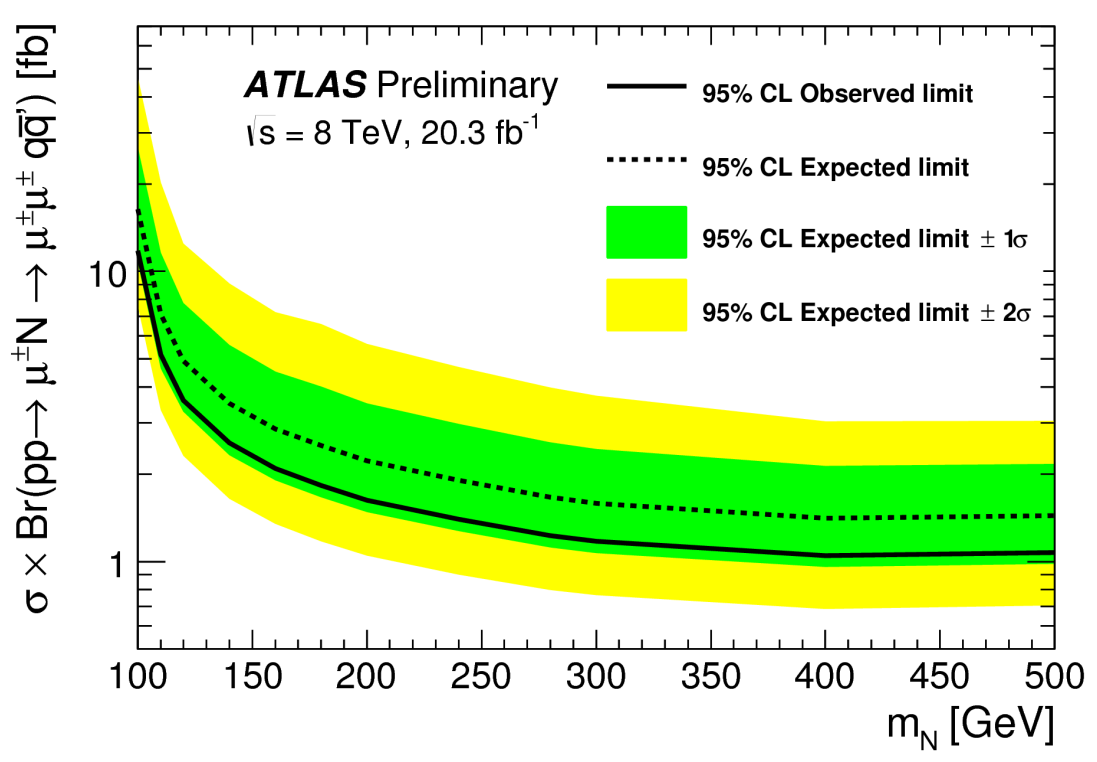

to appear soon on ArXiv
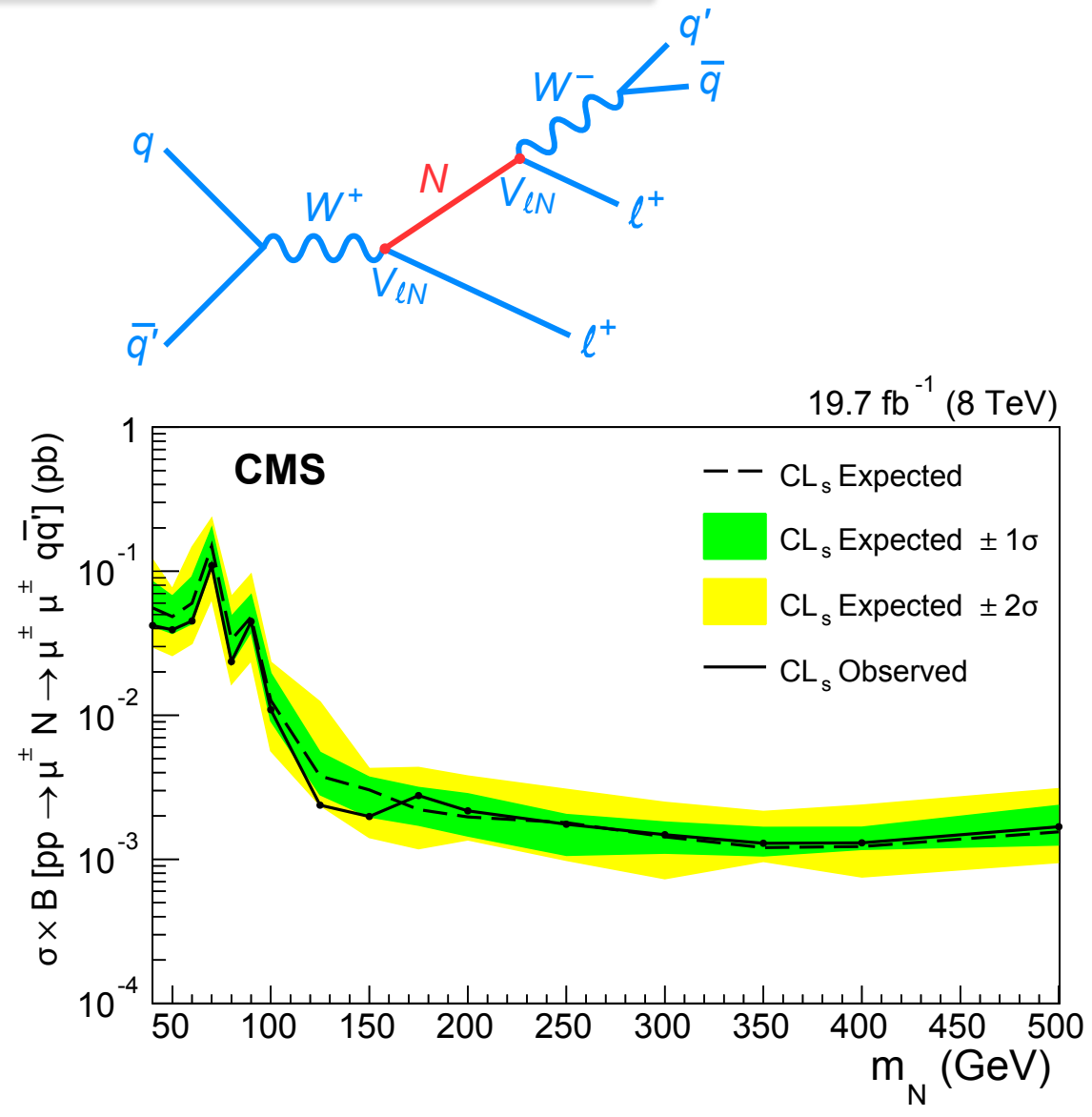

arXiv:1501.05566 


\section{CMS Exotics}

\section{Searches for resonances}

\section{W Combined Results}

Full combination of $X \rightarrow V V$ results in the Bulk Graviton model

$\downarrow$ Improves sensitivity to new physics!

$\downarrow$ Best sensitivity from lepton+ $\mathrm{E}_{T}{ }^{\text {miss }}+\mathrm{V}$-jet channel over the whole mass range, followed by the allhadronic channel at higher masses

$\downarrow$ Good sensitivity from the 2 leptons+V-jet channel at low masses

$\downarrow$ Interesting deviation from expected background $(1.3 \sigma)$ in all channels at $\mathrm{M} \sim 1.8-2 \mathrm{TeV}$ !

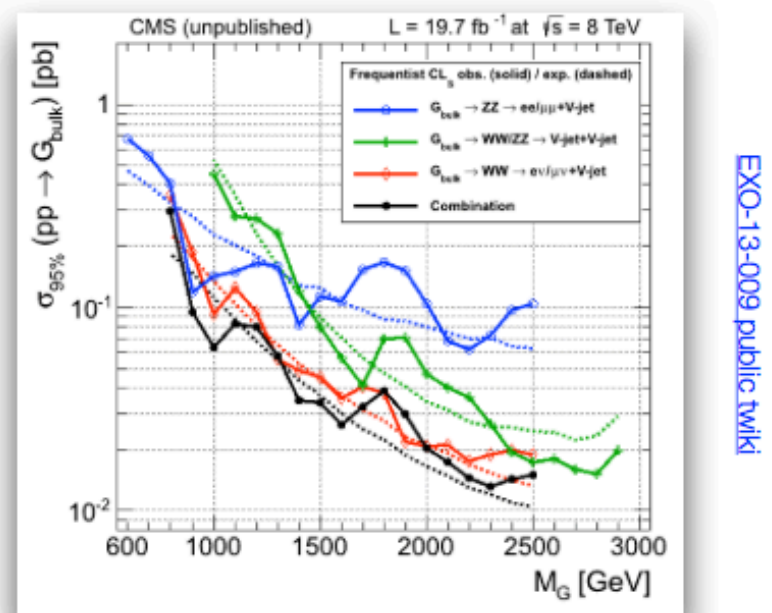




\section{CMS Higgs excess}

\section{- CMS LFV $\mathrm{H} \rightarrow \mu \tau$}

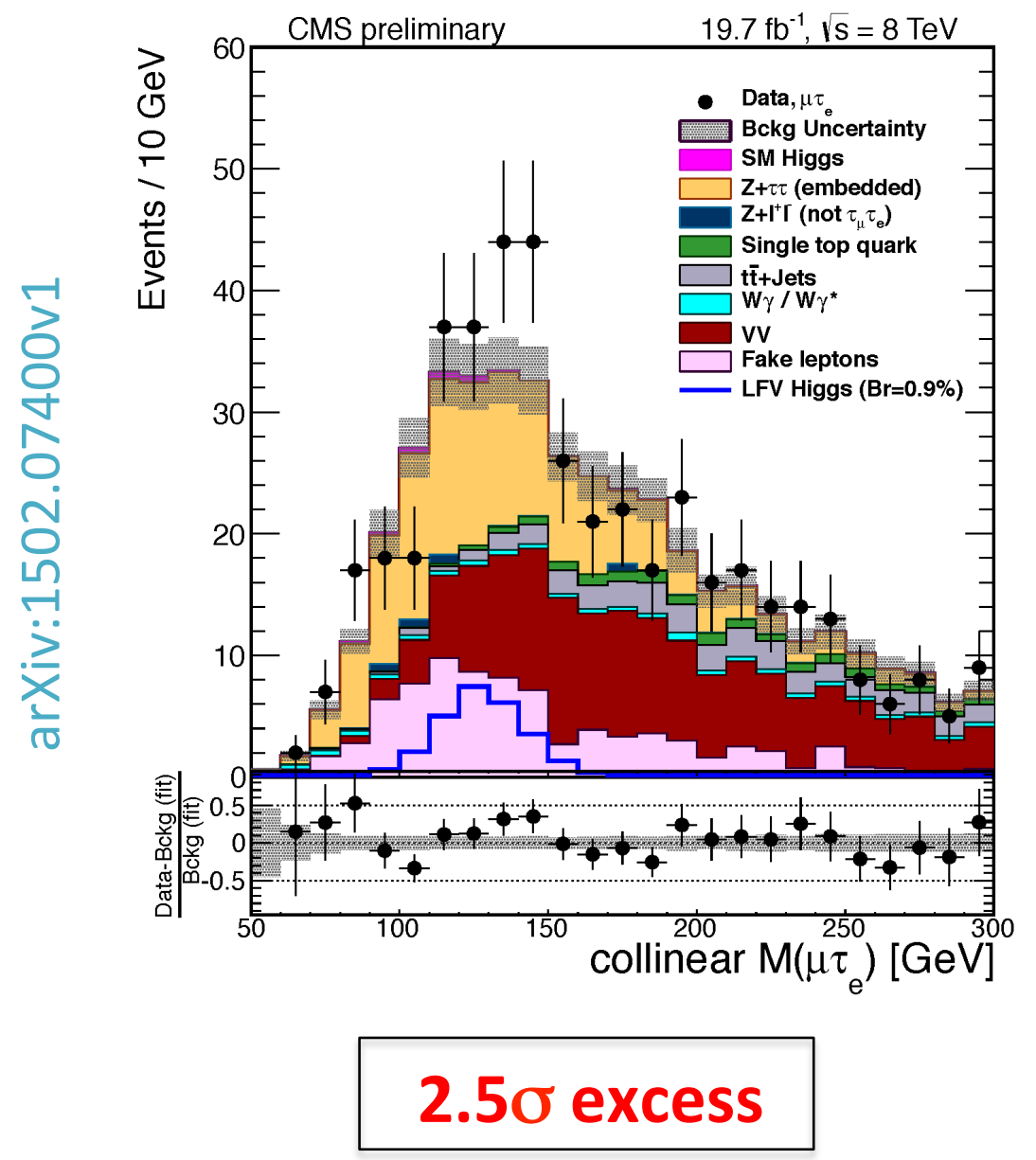

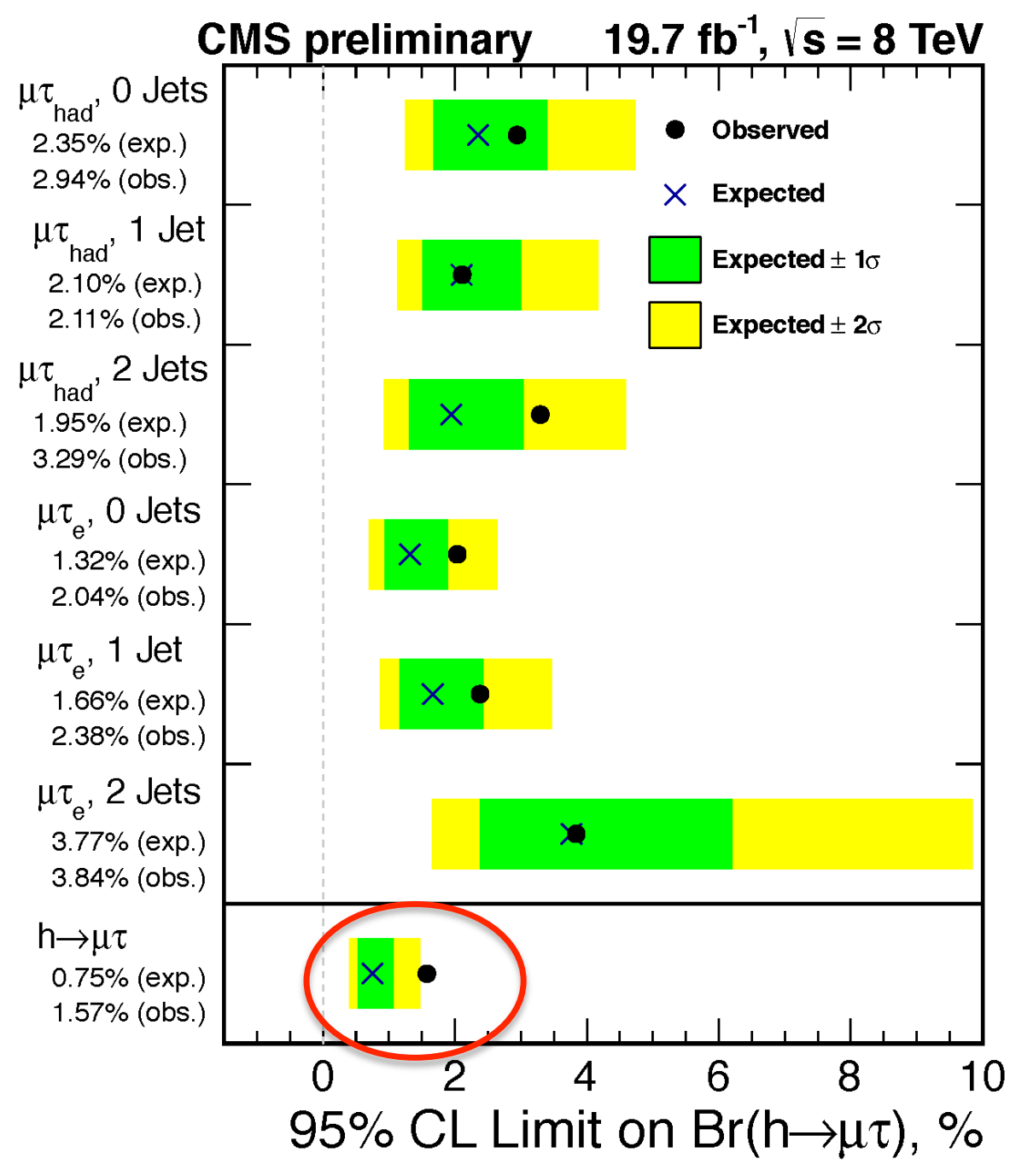




\section{ATLAS Exotics Excess}

\section{Type III See-Saw heavy leptons}

in $2 \mathrm{~L}+2 \mathrm{~J}$ final state :

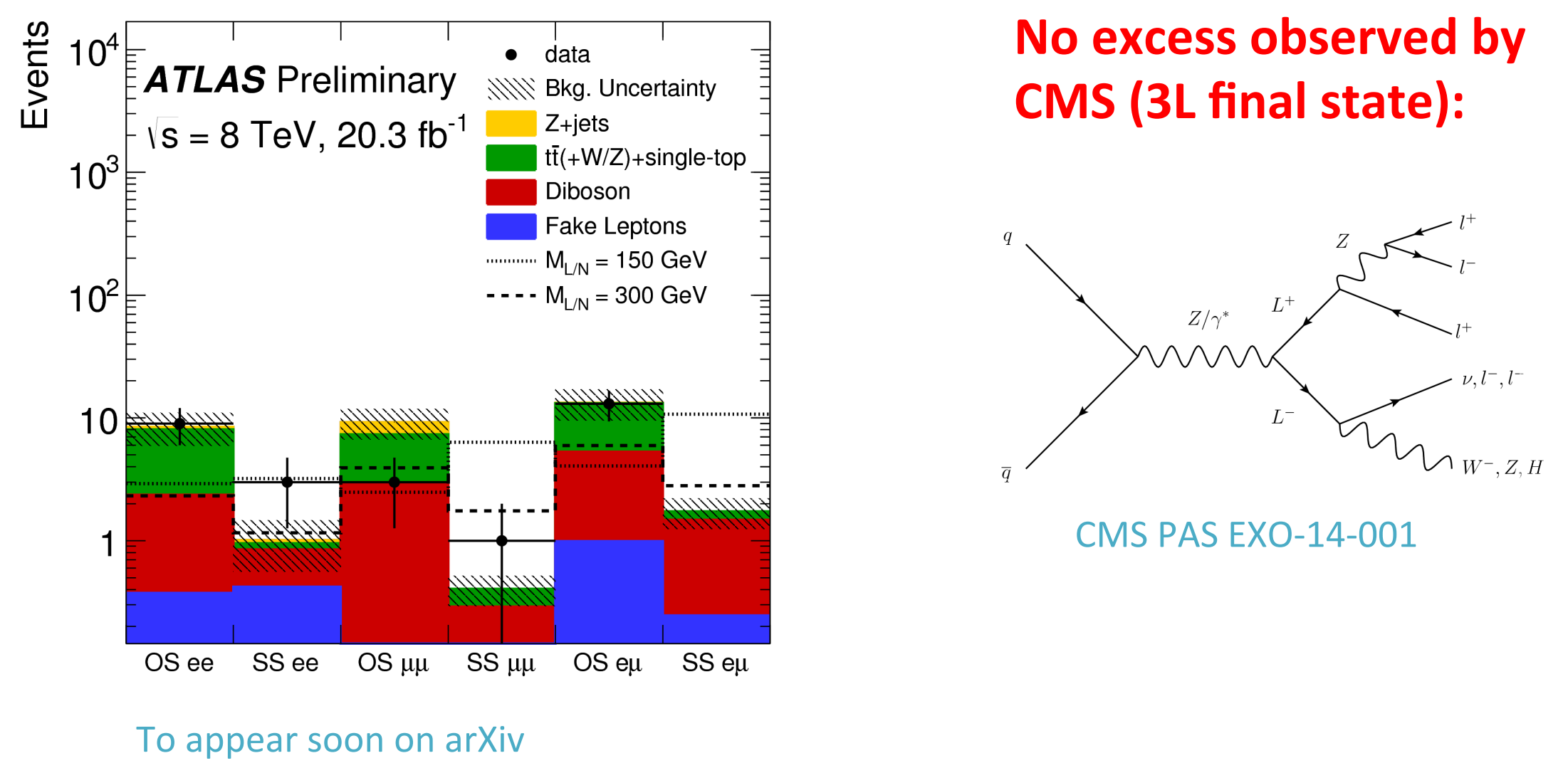




\section{ATLAS Higgs excess}

- Di-Higgs resonances $\quad G^{*} \rightarrow H H \rightarrow b \bar{b} b \bar{b}$ (or $b \bar{b} \gamma \gamma$ )

bbyy final state :

Small statistics
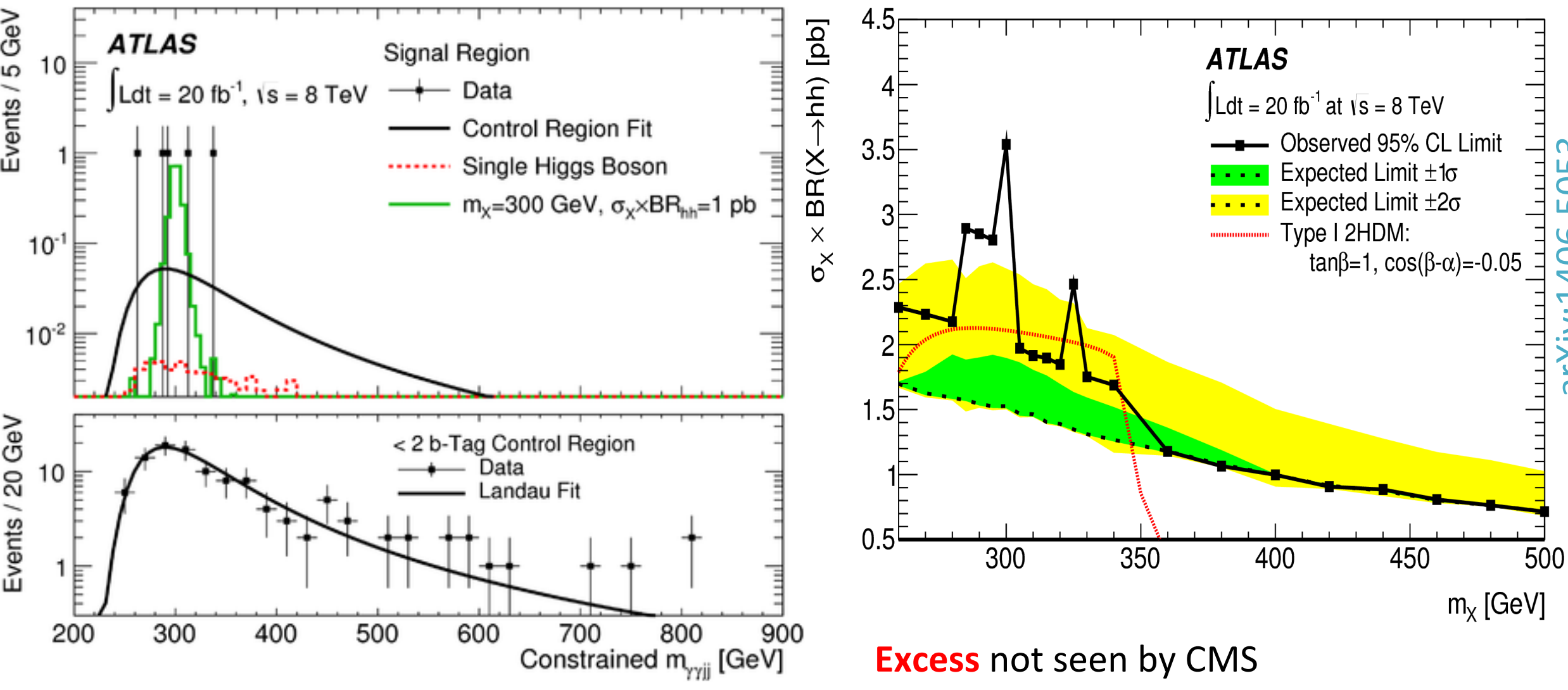

Excess not seen by CMS

(CMS PAS HIG-13-032) 


\section{CMS SUSY Excess}

\section{Multilepton $31+\tau$}

- Right-handed stau lepton is (N)NLSP

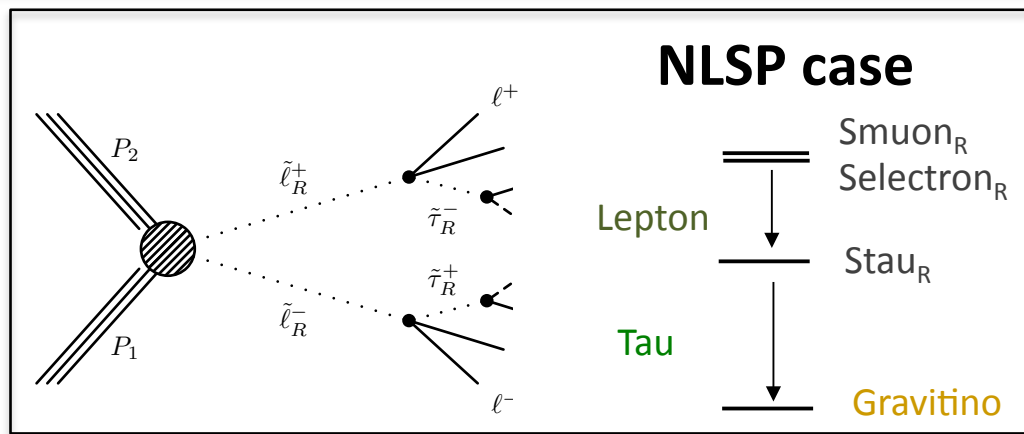

- Signal regions with off-shell Z, hadronic taus without b-jets

\begin{tabular}{|r|c|c|c|c|}
\hline $\begin{array}{r}\text { Missing energy } \\
(\mathrm{GeV})\end{array}$ & $\begin{array}{c}\mathbf{H}_{\mathrm{T}}>\mathbf{2 0 0 \mathrm { GeV } ,} \\
\text { observed }\end{array}$ & $\begin{array}{c}\mathbf{H}_{\mathrm{T}}>\mathbf{2 0 0 G e V} \\
\text { expected }\end{array}$ & $\begin{array}{c}\mathbf{H}_{\mathrm{T}}<200 \mathrm{GeV}, \\
\text { observed }\end{array}$ & $\begin{array}{c}\mathbf{H}_{\mathrm{T}}<200 \mathrm{GeV}, \\
\text { expected }\end{array}$ \\
\hline$>\mathbf{1 0 0}$ & 1 & $0.25 \pm 0.11$ & 3 & $0.60 \pm 0.24$ \\
\hline$[\mathbf{5 0 , 1 0 0 ]}$ & 1 & $0.29 \pm 0.13$ & 4 & $2.1 \pm 0.5$ \\
\hline$[\mathbf{0 , 5 0}]$ & 0 & $0.27 \pm 0.12$ & 15 & $7.5 \pm 2.0$ \\
\hline
\end{tabular}

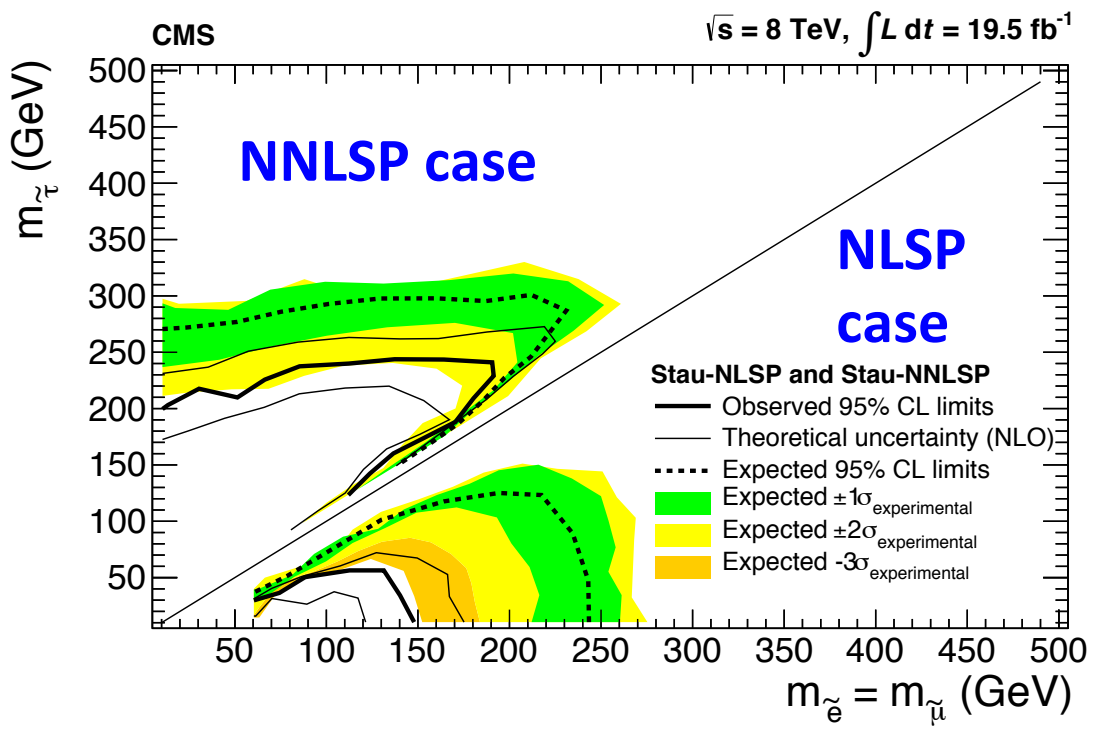

\section{$3 \sigma$ for NLSP case \\ $2 \sigma$ for NNLSP case}




\section{Run1 : ATLAS SUSY searches}

ATLAS SUSY Searches* - 95\% CL Lower Limits

ATLAS Preliminary Status: Feb 2015

$e, \mu, \tau, \gamma$ Jets $E_{\mathrm{T}}^{\mathrm{miss}} \int \mathcal{L} d t\left[\mathrm{fb}^{-1}\right]$

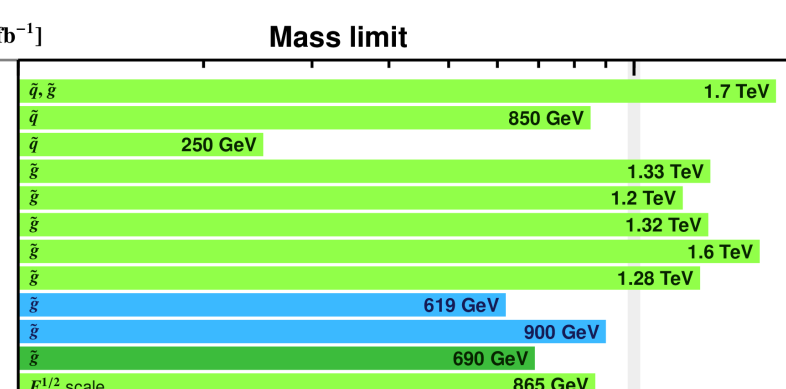

$\tilde{q} \tilde{q}, \tilde{q} \rightarrow q \tilde{X}_{1}^{0}$

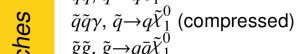

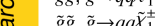

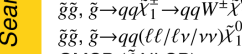

$.2 \mathrm{GMSB}(\tilde{e} \mathrm{NLSP})$

GGM (bino NLSP)

GGM (higgsino-bino NLSP)

GGM (higgsino NLSP)

Gravitino LSP

\begin{tabular}{|c|c|c|c|}
\hline & Gravitino LSP & 0 & mono-jet \\
\hline \multirow{4}{*}{ 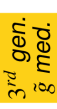 } & $\tilde{g} \rightarrow b \bar{b} \tilde{\chi}_{1}^{0}$ & 0 & $3 b$ \\
\hline & $\tilde{g} \rightarrow t \overline{x_{1}} \tilde{x}_{1}^{0}$ & 0 & $7-10$ jets \\
\hline & $\tilde{g} \rightarrow t \tilde{\bar{\chi}} \tilde{x}_{1}^{0}$ & $0-1 e, \mu$ & $3 b$ \\
\hline & $\tilde{g} \rightarrow b \tilde{t} \tilde{X}_{1}^{+}$ & $0-1 e, \mu$ & $3 b$ \\
\hline
\end{tabular}

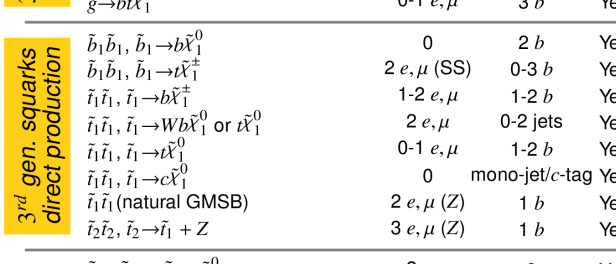

\begin{tabular}{|c|c|c|c|}
\hline & $\tilde{t}_{2} \tilde{t}_{2}, \tilde{t}_{2} \rightarrow \tilde{t}_{1}+Z$ & $3 e, \mu(Z)$ & $1 b$ \\
\hline \multirow{7}{*}{ w } & $\tilde{\ell}_{\mathrm{L}, \mathrm{R}} \tilde{\ell}_{\mathrm{L}, \mathrm{R}}, \tilde{\ell} \rightarrow \ell \tilde{\chi}_{1}^{0}$ & $2 e, \mu$ & 0 \\
\hline & $\tilde{\chi}_{1}^{+} \tilde{\chi}_{1}^{-}, \tilde{\chi}_{1}^{+} \rightarrow \tilde{\ell} v(\ell \tilde{v})$ & $2 e, \mu$ & 0 \\
\hline & $\tilde{\chi}_{1}^{+} \tilde{\chi}_{1}^{-}, \tilde{\chi}_{1}^{+} \rightarrow \tilde{\tau} v(\tau \tilde{v})$ & $2 \tau$ & \\
\hline & $\tilde{\chi}_{1}^{ \pm} \tilde{\chi}_{2}^{0} \rightarrow \tilde{\ell}_{\mathrm{L}} \nu \tilde{\ell}_{\mathrm{L}} \ell(\tilde{v} v), \ell \tilde{v} \tilde{L}_{\mathrm{L}} \ell(\tilde{v} v)$ & $3 e, \mu$ & 0 \\
\hline & $\tilde{\chi}_{1}^{ \pm} \tilde{\chi}_{2}^{0} \rightarrow W \tilde{\chi}_{1}^{0} Z \tilde{x}_{1}^{0}$ & $2-3 e, \mu$ & $0-2$ jets \\
\hline & $\tilde{\chi}_{1}^{ \pm} \tilde{\chi}_{2}^{0} \rightarrow W \tilde{\chi}_{1}^{0} h \tilde{\chi}_{1}^{0}, h \rightarrow b \bar{b} / W W / \tau \tau / \gamma \gamma$ & $e, \mu, \gamma$ & $0-2 b$ \\
\hline & $\tilde{\chi}_{2}^{0} \tilde{\chi}_{3}^{0}, \tilde{\chi}_{2,3}^{0} \rightarrow \tilde{\ell}_{\mathrm{R}} \ell$ & $4 e, \mu$ & 0 \\
\hline
\end{tabular}

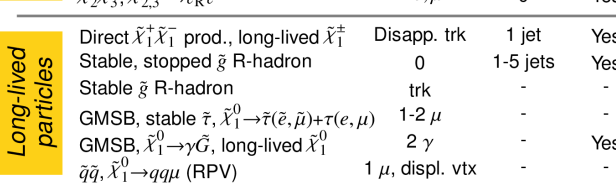

LFV $p p \rightarrow \tilde{v}_{\tau}+X, \tilde{v}_{\tau} \rightarrow e+\mu \quad 2 e, \mu$

$\operatorname{LFV} p p \rightarrow \tilde{v}_{\tau}+X, \tilde{v}_{\tau} \rightarrow e(\mu)+\tau \quad 1 e, \mu+\tau$

$\begin{array}{lc}\text { Bilinear RPV CMSSM } & 2 e, \mu(\mathrm{SS}) \\ \tilde{\chi}_{1}^{\prime} \tilde{\chi}_{1}^{-}, \tilde{\chi}_{1}^{+} \rightarrow W \tilde{\chi}_{1}^{0}, \tilde{\chi}_{1}^{0} \rightarrow e e \tilde{v}_{\mu}, e \mu \tilde{v}_{e} & 4 e, \mu\end{array}$

$\begin{array}{cc}1 e, \mu+\tau & - \\ 2 e, \mu \text { (SS) } & 0-3 b\end{array}$

$\tilde{\chi}_{1}^{+} \tilde{\chi}_{1}^{-}, \tilde{\chi}_{1}^{+} \rightarrow W \tilde{\chi}_{1}^{0}, \tilde{\chi}_{1}^{0} \rightarrow \tau \tau \tilde{v}_{e}, e \tau \tilde{\nu}_{\tau} \quad 3 e, \mu+\tau$

$\tilde{g} \rightarrow q q q$

$\tilde{s} \rightarrow \tilde{t}_{1} t, \tilde{t}_{1} \rightarrow b s$

\begin{tabular}{cccc} 
& Yes & 20.3 \\
0 & $6-7$ jets & Yes & 20.3 \\
\hline & - & 20.3
\end{tabular}

\begin{tabular}{l|l}
\hline & $F$ \\
\hline & $\tilde{g}$
\end{tabular}

\begin{tabular}{ll}
\hline$\tilde{g}$ \\
$\tilde{g}$ \\
$\tilde{g}$
\end{tabular}

$690 \mathrm{Ge}$

$865 \mathrm{GeV}$

$\sqrt{s}=7,8 \mathrm{TeV}$

Reference

Other Scalar charm, $\tilde{c} \rightarrow \tilde{X}_{1}^{0}$

$\sqrt{s}=7 \mathrm{TeV}$ $\begin{array}{llll}2 e, \mu \text { (SS) } \quad 0-3 b & \text { Yes } \quad 20.3\end{array}$

$\sqrt{s}=8 \mathrm{TeV}$

$2 c \quad$ Yes
$\sqrt{s}=8 \mathrm{TeV}$

$\begin{array}{ll}\sqrt{s}=8 \mathrm{Te} \\ \text { partial data } & \text { full data }\end{array}$

20.3

$\begin{array}{lcc}\tilde{g} & & 1.25 \mathrm{TeV} \\ \tilde{g} & & 1.1 \mathrm{TeV} \\ \tilde{g} & & 1.34 \mathrm{TeV} \\ \tilde{g} & & 1.3 \mathrm{TeV} \\ \tilde{b}_{1} & & \\ \tilde{b}_{1} & 100-620 \mathrm{GeV} & \\ \tilde{t}_{1} 110-167 \mathrm{GeV} & 275-440 \mathrm{GeV} & \\ \tilde{r}_{1} & 230-460 \mathrm{GeV} & \end{array}$

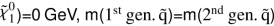

$m(\tilde{q})-m\left(\tilde{X}_{1}^{0}\right)=m(c)$

1405.7875

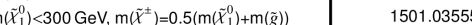

$\mathrm{m}\left(\tilde{\chi}_{1}\right)=0 \mathrm{GeV}$

$\tan \beta>20$

$m\left(x_{1}^{0}\right)>50 \mathrm{GeV}$

$\mathrm{m}\left(\chi_{1}\right)>50 \mathrm{GeV}$

$m(N L S P)>200 \mathrm{GeV}$

$\mathrm{m}(\tilde{G})>1.8 \times 10^{-4} \mathrm{eV}, \mathrm{m}(\tilde{g})=\mathrm{m}(\tilde{q})=1.5 \mathrm{TeV}$

$m\left(\tilde{X}_{10}^{0}\right)<400 \mathrm{GeV}$

$\mathrm{m}\left(\tilde{X}^{0}\right)<300 \mathrm{GeV}$

$\mathrm{m}\left(\tilde{X}_{1}^{0}\right)<90 \mathrm{GeV}$

$\mathrm{m}\left(\tilde{X}_{1}^{ \pm}\right)=2 \mathrm{~m}\left(\tilde{X}_{1}^{0}\right)$

$\mathrm{m}\left(\tilde{X}_{1}^{ \pm}\right)=2 \mathrm{~m}\left(\tilde{X}_{1}^{0}\right), \mathrm{m}\left(\tilde{X}_{1}^{0}\right)=55 \mathrm{GeV}$

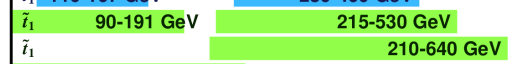

$\mathrm{m}\left(\tilde{X}_{1}\right)=1 \mathrm{GeV}$

$\mathrm{m}\left(\tilde{\tilde{z}}_{1}\right)-\mathrm{m}\left(\tilde{\chi}_{1}^{0}\right)<85 \mathrm{GeV}$

$\begin{array}{ll}\tilde{t}_{1} & 150-580 \mathrm{GeV} \\ \tilde{t}_{2} & 290-600 \mathrm{GeV}\end{array}$

$\mathrm{m}\left(\tilde{X}_{1}^{0}\right)<200 \mathrm{GeV}$

$\begin{array}{lc}\tilde{\tau} & 90-325 \mathrm{GeV} \\ \tilde{\chi}_{1}^{ \pm} & 140-465 \mathrm{GeV} \\ \tilde{\chi}_{1}^{ \pm} & 100-350 \mathrm{GeV}\end{array}$

$\begin{array}{ll}\chi_{1} & 100-350 \mathrm{GeV}\end{array}$

$\mathrm{m}\left(\tilde{X}_{1}^{0}\right)=0 \mathrm{GeV}$

$\mathrm{m}\left(\tilde{X}_{1}^{0}\right)=0 \mathrm{GeV}, \mathrm{m}(\tilde{X}, \tilde{v})=0.5\left(\mathrm{~m}\left(\tilde{X}_{1}^{ \pm}\right)+\mathrm{m}\left(\tilde{X}_{1}^{0}\right)\right)$

$\mathrm{m}\left(\tilde{\chi}_{1}^{0}\right)=0 \mathrm{GeV}, \mathrm{m}(\tilde{\bar{\tau}}, \tilde{\hat{\gamma}})=0.5\left(\mathrm{~m}\left(\tilde{\chi}_{1}^{ \pm}\right)+\mathrm{m}\left(\tilde{\chi}_{1}^{0}\right)\right.$

$\begin{array}{ll}x_{1} & \\ \tilde{x}_{1}^{ \pm}, \tilde{\chi}_{2}^{0} \\ \tilde{x}_{1}^{ \pm} \tilde{\chi}_{2}^{0}\end{array}$ $\mathrm{m}\left(\tilde{X}_{1}^{ \pm}\right)=\mathrm{m}\left(\tilde{X}_{2}^{0}\right), \mathrm{m}\left(\tilde{X}_{1}^{0}\right)=0, \mathrm{~m}(\tilde{\mathcal{X}}, \tilde{v})=0.5\left(\mathrm{~m}\left(\tilde{X}_{1}^{ \pm}\right)+\mathrm{m}\left(\tilde{X}_{1}^{0}\right)\right)$

$\mathrm{m}\left(\tilde{X}_{1}^{ \pm}\right)=\mathrm{m}\left(\tilde{X}_{2}^{0}\right), \mathrm{m}\left(\tilde{X}_{1}^{0}\right)=0$, sleptons decoupled

1405.7875
1405.7875

1411.1559

1501.03555

1501.03555

ATLAS-CONF-2014-001

ATLAS-CONF-2012-144

1211.1167

ATLAS-CONF-2012-152 1502.01518

1407.0600
1308.184

1407.0600 1407.0600

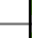

$\begin{array}{ll}\chi_{2,3} & 620 \mathrm{GeV} \\ \tilde{X}_{1}^{ \pm} & 270 \mathrm{GeV}\end{array}$
$\left(\tilde{x}_{3}^{0}\right), m\left(\tilde{X}_{1}^{0}\right)=0, m(\tilde{\ell}, \tilde{y})=0.5\left(m\left(\tilde{x}_{2}^{0}\right)+m\left(\tilde{x}_{1}^{0}\right)\right.$

1308.2631

1404.2500

$1209.2102,1407.0583$

$1403.4853,1412.4742$

$1407.0583,1406.1122$

1407.0608 1403.5222

1403.5294

1403.5294

1407.0350 1402.7029

03.5294, 1402.7029 1501.07110
14055086

$m\left(\tilde{X}_{1}^{ \pm}\right)-m\left(\tilde{X}_{1}^{0}\right)=160 \mathrm{MeV}, \tau\left(\tilde{X}_{1}^{ \pm}\right)=0.2 \mathrm{~ns}$

$\mathrm{m}\left(\tilde{x}_{1}^{0}\right)=100 \mathrm{GeV}, 10 \mu \mathrm{s}<\tau(\tilde{g})<1000 \mathrm{~s}$

1310.6584

$10<\tan \beta<50$

$2<\tau\left(\tilde{X}_{1}^{0}\right)<3$ ns, SPS8 model

$1.0 \mathrm{TeV}$ $1.5<c \tau<156 \mathrm{~mm}, \mathrm{BR}(\mu)=1, \mathrm{~m}\left(\boldsymbol{X}_{1}^{0}\right)=108 \mathrm{GeV}$

141.0705

1409.5542

ATLAS-CONF-2013-092

$\begin{array}{lrr} & 1.0 \mathrm{TeV} & \\ \tilde{q} & & 1.61 \mathrm{TeV}\end{array}$

$\lambda_{311}^{\prime}=0.10, \lambda_{132}=0.05$

$x_{311}^{\prime}=0.10, \lambda_{1(2) 33}=0.05$

$\mathrm{m}(\tilde{q})=\mathrm{m}(\tilde{g}), c \tau_{L S P}<1 \mathrm{~mm}$

$\mathrm{m}\left(\tilde{X}_{1}^{0}\right)>0.2 \times m\left(\tilde{X}_{1}^{ \pm}\right), \lambda_{121} \neq 0$

$\mathrm{m}\left(\bar{X}_{1}\right)>0.2 \times \mathrm{m}\left(\bar{X}_{1}^{\prime}\right), \lambda_{133} \neq 0$

1212.1272

1212.1272

1404.2500

1405.5086

ATLAS-CONF-2013-09 1404.250

$\mathrm{m}\left(\tilde{X}_{1}^{0}\right)<200 \mathrm{GeV}$

1501.01325

*Only a selection of the available mass limits on new states or phenomena is shown. All limits quoted are observed minus $1 \sigma$ theoretical signal cross section uncertainty. 


\section{Run1: CMS SUSY searches (1)}

\section{Summary of CMS SUSY Results* in SMS framework SUSY 2013}

$m$ (mother)-m(LSP) $=200 \mathrm{GeV}$

SUS-13-012 SUS-12-028 L=19.5 $11.7 / \mathrm{fb}$

SUS-12-005 SUS-11-024 L=4.7/fb

SUS-13-004 SUS-12-024 SUS-12-028 L=19.3 19.4 /fb

SUS-13-004 SUS-13-007 SUS-13-008 SUS-13-013 L=19.4 19.5 /fb

SUS-11-011 L=4.98 /fb

SUS-12-004 $L=4.98 / \mathrm{fb}$

$\widetilde{g} \rightarrow q q(\tilde{\alpha} \rightarrow \tau \tau \tilde{\chi} \tilde{\alpha})$

$\tilde{\mathrm{g}} \rightarrow \mathrm{qq}\left(\tilde{\bar{\chi}}^{ \pm} \rightarrow \mathrm{W} \tilde{\chi}^{0} \tilde{\chi}^{0}\right)$

$\tilde{\mathrm{g}} \rightarrow \mathrm{t}(\tilde{\mathrm{t}} \rightarrow \mathrm{t} \tilde{\bar{\chi}})$

$\tilde{g} \rightarrow q q\left(\widetilde{\chi}^{ \pm} \rightarrow I^{ \pm} v \tilde{\chi}^{0}\right.$

$\tilde{\mathrm{g}} \rightarrow \mathrm{qq}\left(\tilde{\chi}_{2}^{0} \rightarrow \mathrm{z} \tilde{\chi}^{0}\right)$

$\tilde{\mathrm{g}} \rightarrow \mathrm{qg}\left(\tilde{\chi}^{ \pm} \rightarrow \mathrm{W} \tilde{\chi}^{0}\right)$

$\tilde{\mathrm{g}} \rightarrow \mathrm{qq}\left(\tilde{\chi}_{2} \rightarrow \gamma \tilde{\chi} \mid \tilde{\chi}^{ \pm} \rightarrow \mathrm{W} \tilde{\chi}\right.$

$\tilde{\mathrm{g}} \rightarrow \mathrm{qq}\left(\widetilde{\chi}_{2}^{0} \rightarrow \gamma \tilde{\chi}^{0}\right)$

$\tilde{\mathrm{g}} \rightarrow \mathrm{b}\left(\tilde{\mathrm{b}} \rightarrow \mathrm{t}\left(\tilde{\chi}^{ \pm} \rightarrow \mathrm{W} \tilde{\chi}^{0}\right)\right)$

SUS-12-010 L=4.98/fb

SUS-11-010 L=4.98 /fb

SUS-11-021 SUS-12-002 L=4.98 $4.73 / \mathrm{fb}$

SUS-13-013 $L=19.5 / \mathrm{fb}$

SUS-12-001 L=4.93/fb

SUS-12-001 L=4.93/fb

SUS-13-008 SUS-13-013 L=19.5/fb

$\widetilde{q} \rightarrow q \tilde{\chi}^{0} \quad$ SUS-13-012 SUS-12-028 L=19.5 $11.7 / \mathrm{fb}$

$\widetilde{q} \rightarrow q \tilde{\chi}^{0} \quad$ SUS-12-005 SUS-11-024 L=4.7/fb

$\tilde{\mathrm{t}} \rightarrow \mathrm{t} \tilde{\chi}^{0} \quad$ SUS-13-011 SUS-13-004 L=19.5 $19.3 / \mathrm{fb}$

$\tilde{\mathrm{t}} \rightarrow \mathrm{t} \tilde{\chi}^{0}$ SUS-11-024 SUS-12-005 L=4.7/fb

$\tilde{\mathrm{t}} \rightarrow \mathrm{b}\left(\tilde{\chi}^{+} \rightarrow \mathrm{W} \tilde{\chi}^{0}\right) \quad$ SUS-13-011 L $=19.5 / \mathrm{fb}$

SUS-13-011 $L=19.5 / \mathrm{tb}$

SUS-11-030 L=4.98 / fb

$\tilde{\mathrm{t}} \rightarrow \mathrm{tb} \tilde{\chi}^{0}\left(\tilde{\chi}^{0} \rightarrow H G\right)$

$-$

$\tilde{b} \rightarrow b \tilde{\chi}^{0}$

$\underset{\tilde{b}}{\tilde{b}} \rightarrow \mathrm{tW} \tilde{\chi}_{0}^{0}$

SUS-12-028 L=11.7/

SUS-13-008 SUS-13-013 L=19.5/fb

SUS-13-008 L=19.5/fb

$\sqrt{s}=7 \mathrm{TeV}$

$\mathrm{b} \rightarrow \mathrm{bZ} \tilde{\chi}$

$\tilde{\chi}_{2}^{0} \tilde{\chi}^{ \pm} \rightarrow I I I v \tilde{\chi}^{0} \tilde{\chi}^{0}$

$\tilde{\alpha} \tilde{\alpha} \rightarrow I^{+} I^{-} v \vee \tilde{\chi}^{0} \tilde{\chi}^{0}$

$\tilde{\chi}^{ \pm} \tilde{\chi}_{\text {. }}^{0} \rightarrow \mathrm{wz} \tilde{\chi}_{0}^{0} \tilde{\chi}_{0}^{0}$

$\tilde{\chi}_{0}^{ \pm} \tilde{x}_{2} \rightarrow \mathrm{HW} \tilde{\chi}^{0} \tilde{x}^{0}$

$\tilde{\chi}^{0} \tilde{\chi}^{2} \rightarrow \| \tau v \tilde{\chi}^{0} \tilde{\chi}$

$\tilde{\chi}_{2}^{02} \tilde{\chi}^{ \pm} \rightarrow \tau \tau \vee \tilde{\chi}^{0} \tilde{\chi}^{0}$

$\bar{\imath}_{\rightarrow 1} \tilde{\chi}^{0}$

SUS-13-006 L=19.5/fb

SUS-13-006 L=19.5/fb

SUS-13-006 L=19.5/fb

SUS-13-017 L=19.5/fb

SUS-13-006 L=19.5/fb $\quad x=0.05 \quad x=0.50$

SUS-13-006 L=19.5/fb

/fb

$\sqrt{s}=8 \mathrm{TeV}$

200

$\frac{1}{400}$

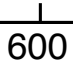

$\frac{1}{800}$

CMS Preliminary

*Observed limits, theory uncertainties not included

Only a selection of available mass limits

Probe *up to* the quoted mass limit 


\section{Run1: CMS SUSY searches (2)}

Summary of CMS RPV SUSY Results*

EPSHEP 2013

$\tilde{\mathrm{g}} \rightarrow$ qlv $\lambda_{122}$

$\widetilde{\mathrm{g}} \rightarrow$ qlv $\lambda_{123}$

$\widetilde{\mathrm{g}} \rightarrow$ qlk $\lambda_{233}$

$\widetilde{g} \rightarrow$ qbt $\mu \lambda^{\prime}$

$\widetilde{g} \rightarrow q b t \mu \lambda^{231}$

$\widetilde{\mathrm{g}} \rightarrow \mathrm{qqb} \lambda^{\prime \prime}{ }_{113 / 223}$

$\tilde{g} \rightarrow$ qqq $\lambda^{11}$

$\widetilde{\mathrm{g}} \rightarrow \mathrm{tbs} \lambda^{11}{ }^{12}$

$\tilde{\mathrm{g}} \rightarrow$ qqqq $\lambda{ }^{\prime 323}$

$\tilde{q} \rightarrow$ qlh $\lambda_{122}$

$\widetilde{\mathrm{q}} \rightarrow$ qlv $\lambda_{123}$

$\widetilde{q} \rightarrow$ qlv $\lambda_{233}$

$\widetilde{\mathrm{q}} \rightarrow \mathrm{qbt} \mu \lambda^{\prime 23}$

$\widetilde{q} \rightarrow q b t \mu \lambda^{\prime 231}$

$\tilde{\mathrm{q}}_{\mathrm{R}} \rightarrow$ qqqq $\lambda{ }^{233}$

$\tilde{\mathrm{t}}_{\mathrm{R}} \rightarrow$ uevt $\lambda_{122}^{112}$

$\tilde{\mathrm{t}}_{\mathrm{R}} \rightarrow \mu \tau v \mathrm{t} \lambda_{123}$

$\tilde{\mathrm{t}}_{\mathrm{R}} \rightarrow \mu \tau v \mathrm{t} \lambda_{233}$

$\tilde{\mathrm{t}}_{\mathrm{R}} \rightarrow \operatorname{tbt} \mu \lambda^{\prime}$

$\tilde{t} \rightarrow b \tau \lambda^{\prime 233}$

SUS-12-027 L=9.2 /fb

SUS-12-027 L=9.2/fb

SUS-12-027 L=9.2/fb

SUS-12-027 L=9.2/fb

SUS-12-027 L=9.2/fb

EXO-12-049 L=19.5 /fb

EXO-12-049 L=19.5/fb

SUS-13-013 L=19.5/fb

SUS-12-027 L=9.2/fb

SUS-12-027 L=9.2/fb

SUS-12-027 L=9.2/fb

SUS-12-027 L=9.2/fb

SUS-12-027 L=9.2/fb

SUS-12-027 L=9.2/fb

SUS-12-027 L=9.2 /fb

SUS-13-003 L=19.5 9.2 /fb

SUS-12-027 L=9.2/fb

$\sqrt{\mathrm{s}}=7 \mathrm{TeV}$

SUS-13-003 L=19.5 9.2 /fb

SUS-13-003 L=19.5 /fb

$\sqrt{s}=8 \mathrm{TeV}$

CMS Preliminary

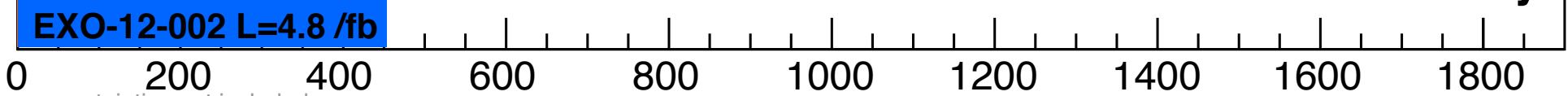
Mass scales $[\mathrm{GeV}] 60$ 


\section{Run1 : ATLAS Exotics searches (1)}

ATLAS Exotics Searches* - 95\% CL Exclusion

ATLAS Preliminary Status: March 2015

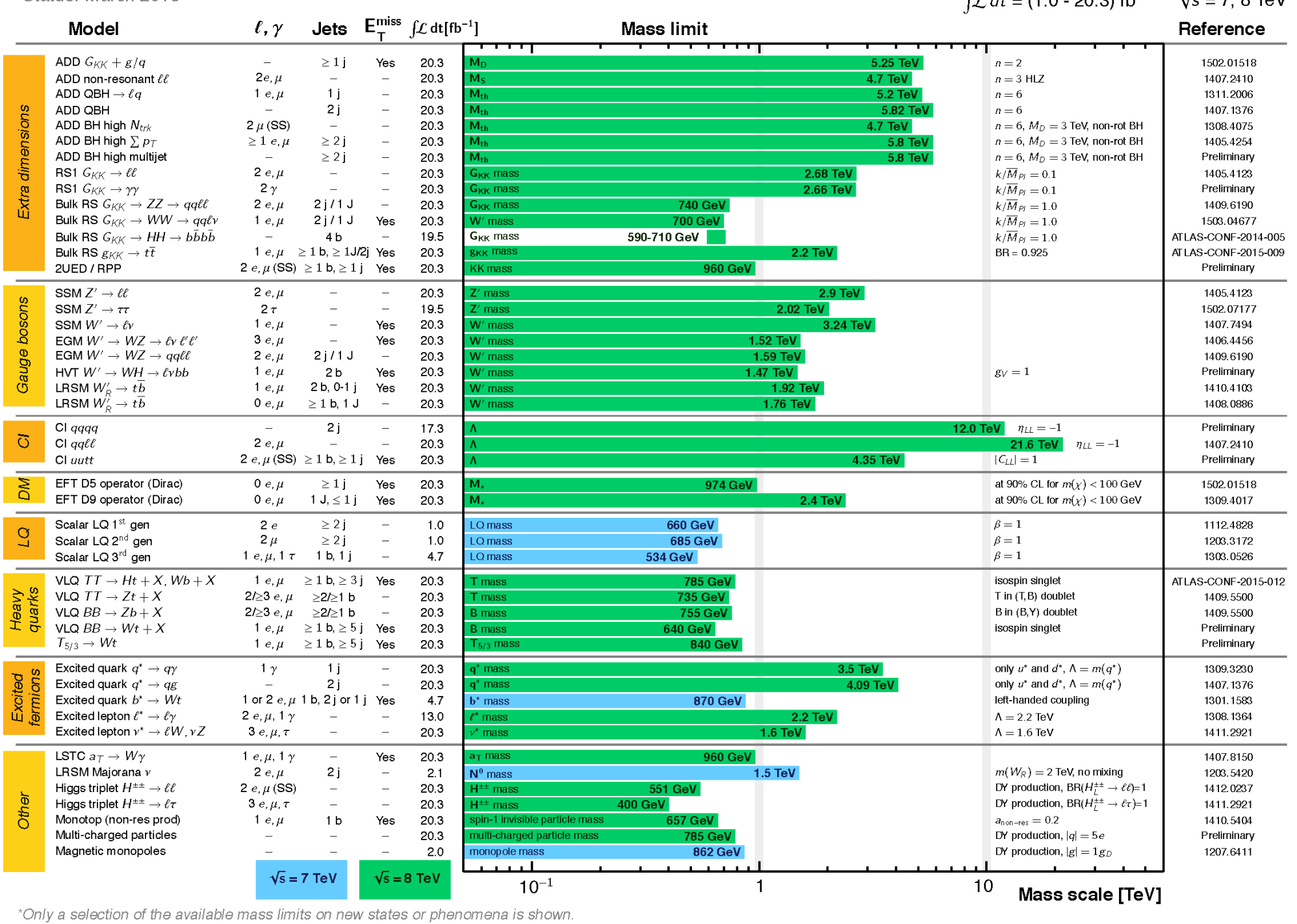




\section{Run1 : ATLAS Exotics searches (2)}

ATLAS Exotics Long-lived Particle Searches* - 95\% CL Exclusion

ATLAS Preliminary Status: March 2015

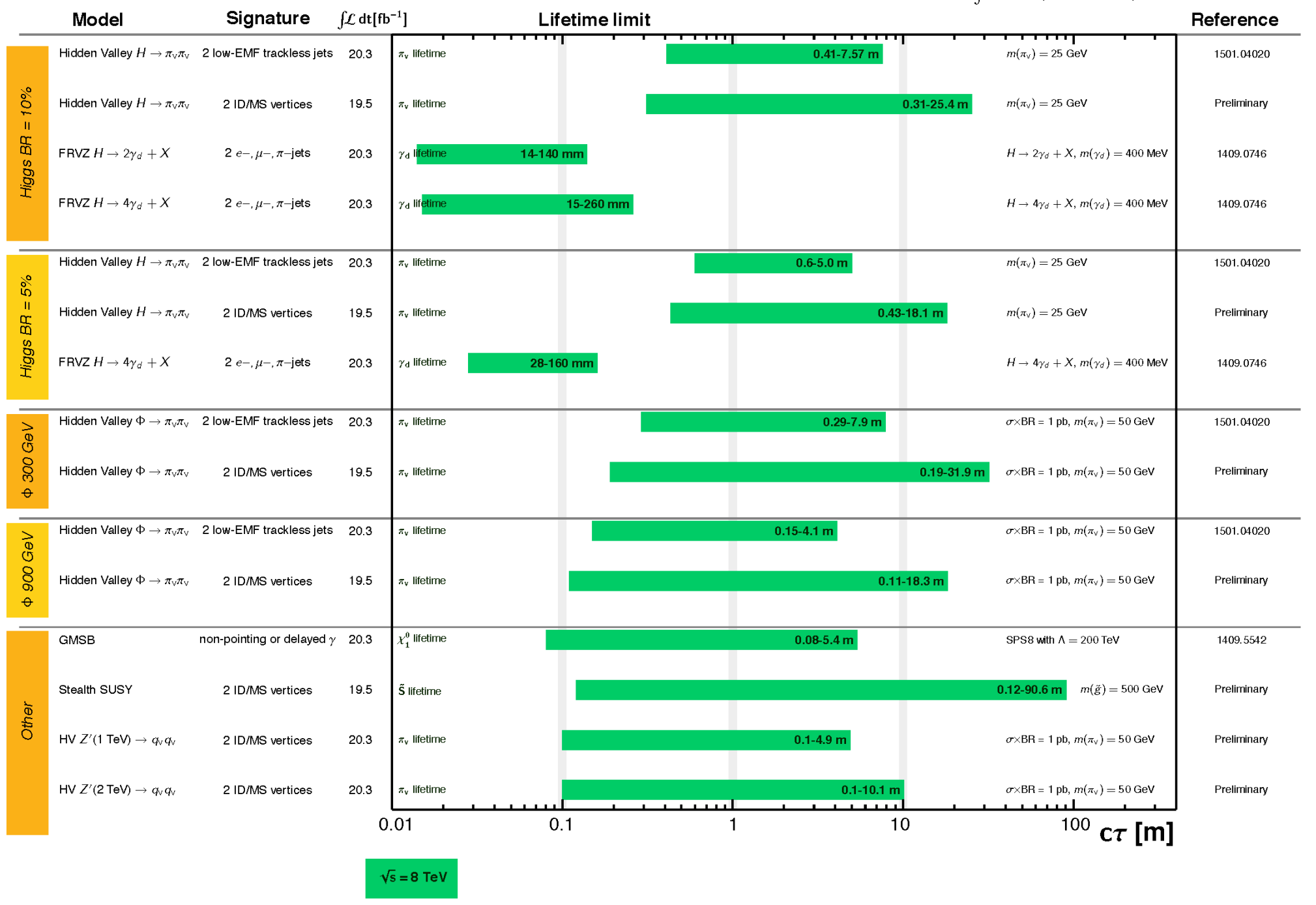




\section{Run1: CMS Exotics searches (2)}

CMS Searches for New Physics Beyond Two Generations (B2G)

95\% CL Exclusions (TeV)
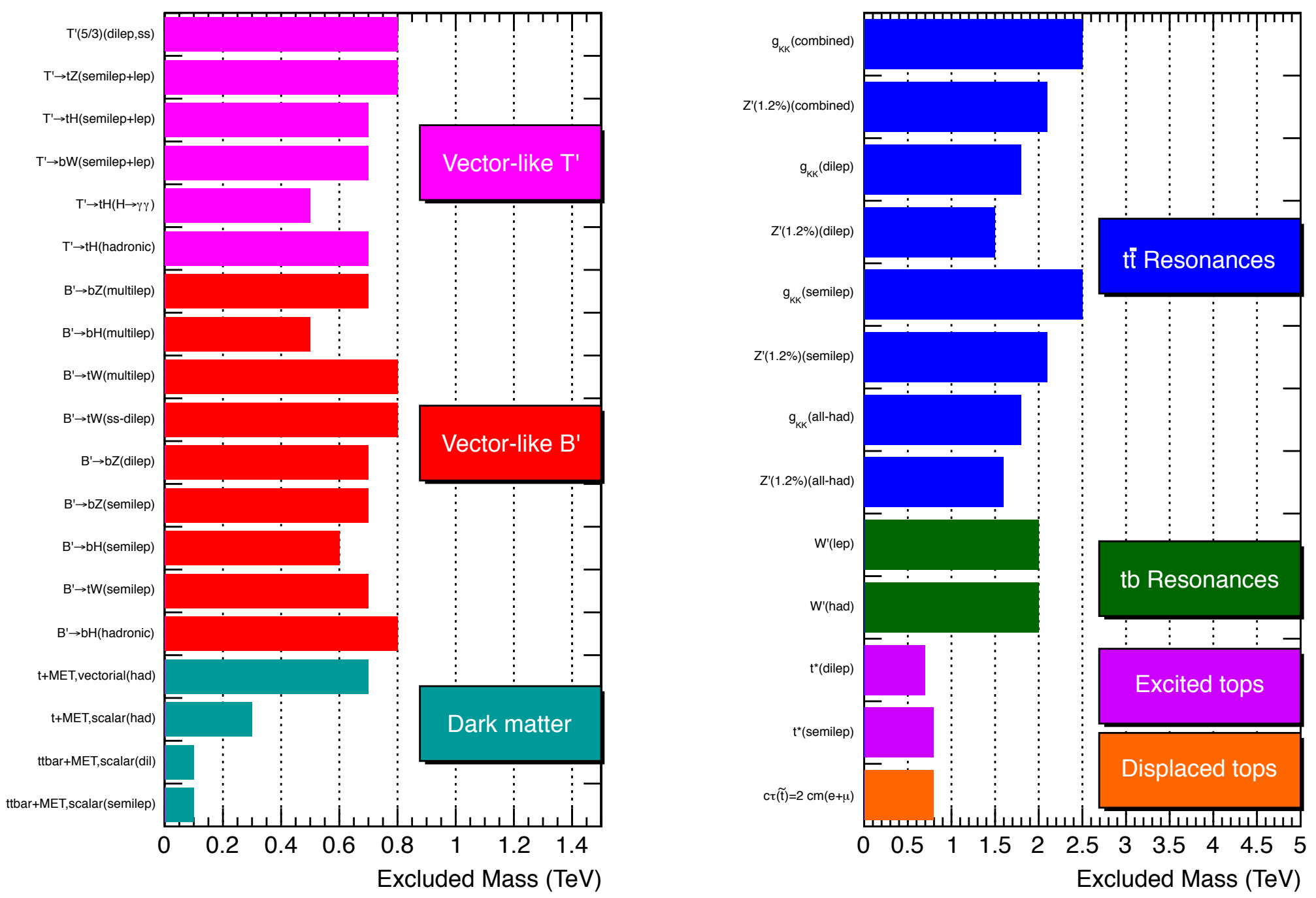


\section{Run1 : Limits on SUSY (1)}

\section{- Strong production}

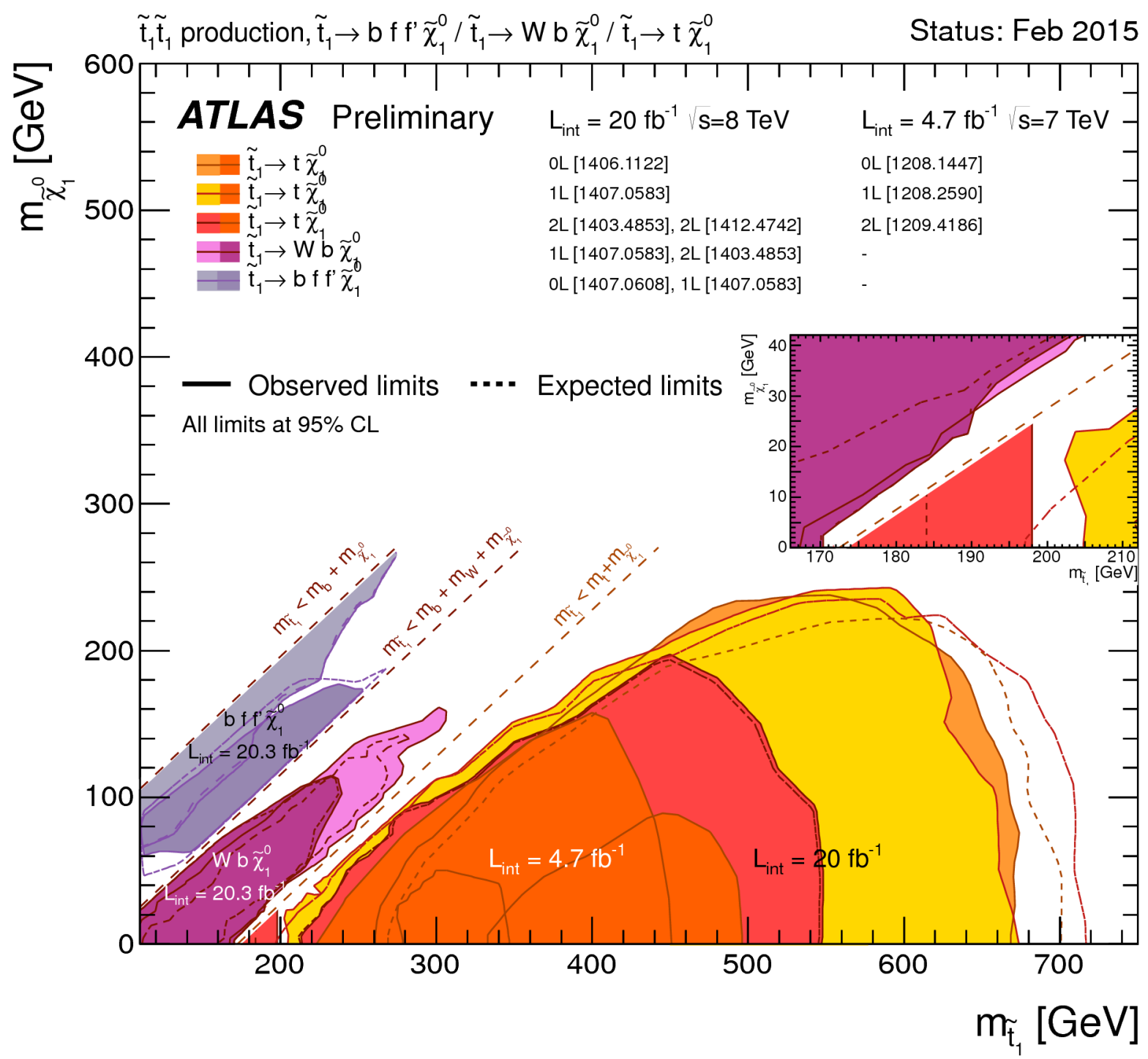




\section{CMS Exotic Discovery Reach : Run3/HL-LHC}

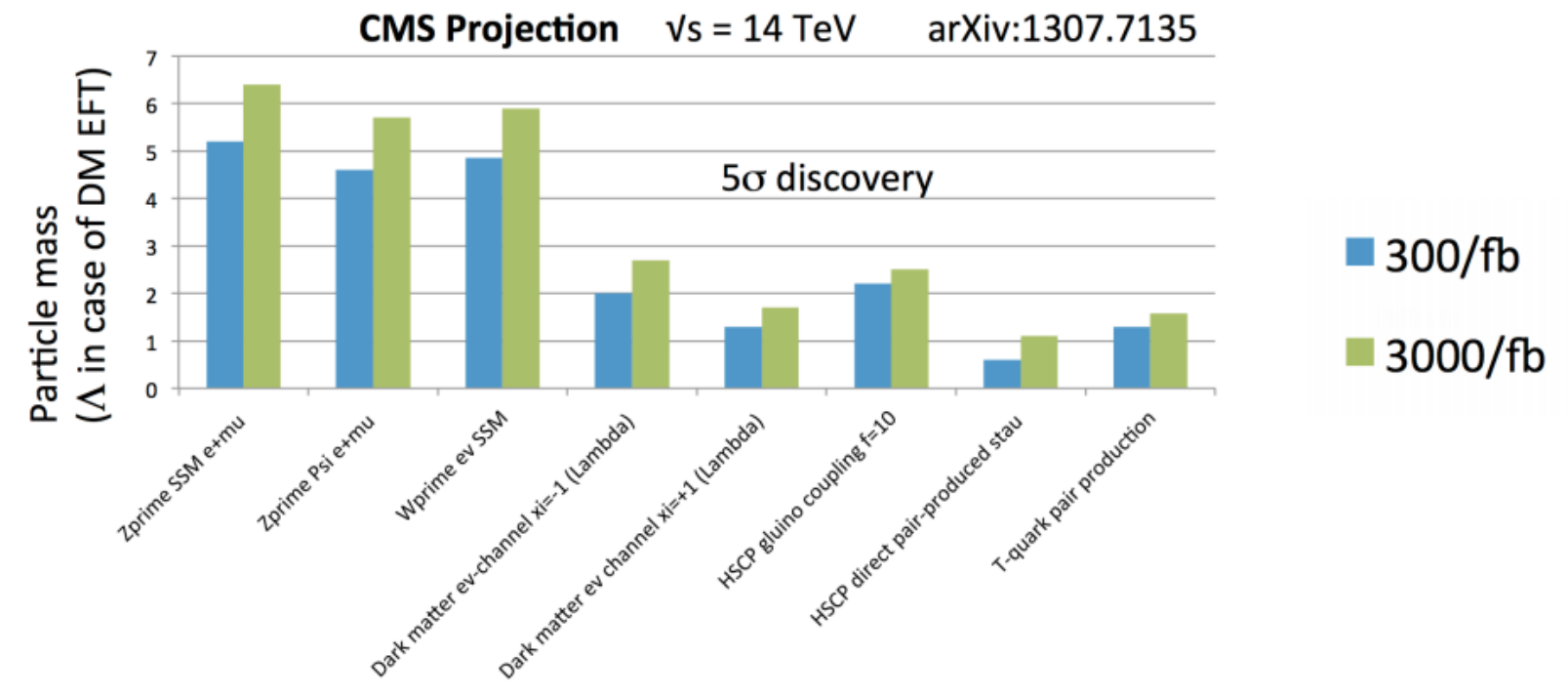

For the EFT description of pair-produced dark matter, the interesting parameter is the cut-off scale $\Lambda=M / \operatorname{sqrt}\left(g_{D M} g_{S M}\right)$ with $M$ being the mediator mass (assumed to be high) and $\mathrm{g}$ being the DM and SM couplings, respectively. The chosen dark matter monolepton channel allows to study potentially different couplings to up- and down-type quarks parametrized by xi.
Projected performance of selected BSM searches with an upgraded $\mathrm{CMS}$ detector at the LHC and HL-LHC (arXiv: 1307.7135).

Except for dark matter, the 5 sigma discovery reach in terms of particle mass is shown. Selected models include new resonances $Z^{\prime}$ and $\mathrm{W}^{\prime}$, heavy stable charged particles (HSCP) such as gluino or stau, and pairproduced T-quarks. 


\section{Run2 (and further) : Higgs searches}

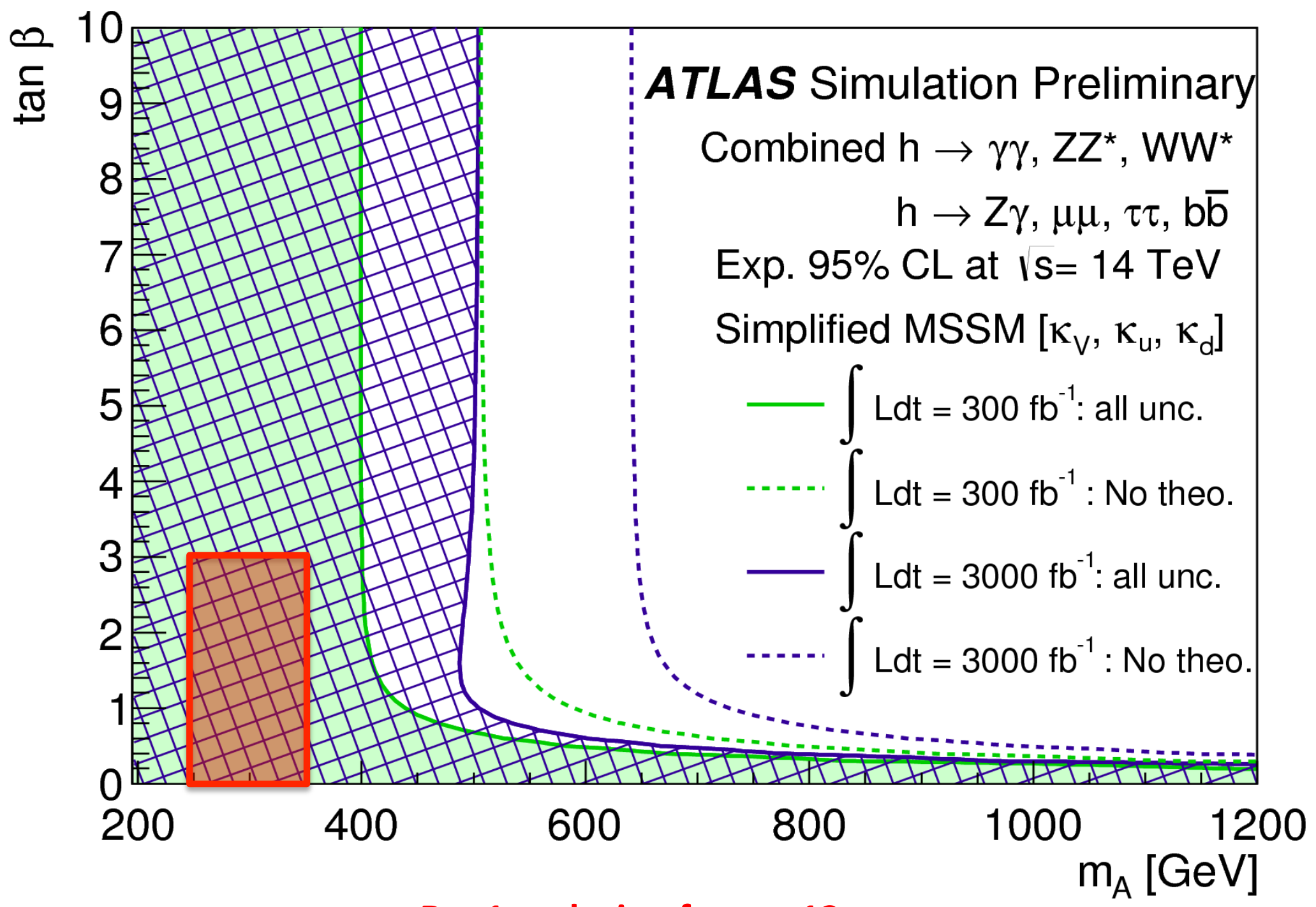

